\title{
Local B-model and mixed Hodge
}

\section{structure}

\author{
Yukiko Konishi ${ }^{1}$ and Satoshi Minabe ${ }^{2}$ \\ ${ }^{1}$ Department of Mathematics, Kyoto University, Kyoto 606-8502, Japan \\ konishi@math.kyoto-u.ac.jp \\ ${ }^{2}$ Department of Mathematics, Tokyo Denki University, 101-8457 Tokyo, \\ Japan \\ minabe@mail.dendai.ac.jp
}

\begin{abstract}
We study the mixed Hodge theoretic aspects of the B-model side of local mirror symmetry. Our main objectives are to define an analogue of the Yukawa coupling in terms of the variations of the mixed Hodge structures and to study its properties. We also describe a local version of Bershadsky-Cecotti-Ooguri-Vafa's holomorphic anomaly equation.
\end{abstract}

\section{Introduction}

\subsection{Local mirror symmetry}

Mirror symmetry states a relationship between the genus zero GromovWitten (GW) theory ("A-model") of a Calabi-Yau threefold $X$ and the Hodge theory ("B-model") of its mirror Calabi-Yau threefold $X^{\vee}$. After the first example of a quintic hypersurface in $\mathbb{P}^{4}$ and its mirror $[9,18]$, Batyrev [6] showed that a mirror pair of Calabi-Yau hypersurfaces in toric varieties can

e-print archive: http://lanl.arXiv.org/abs/0907.4108 

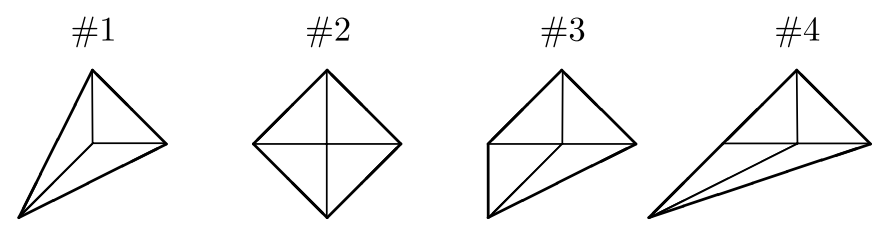

Figure 1: Examples of two-dimensional reflexive polyhedra. $\left(\mathbb{P}^{2}, \mathbb{F}_{0}, \mathbb{F}_{1}, \mathbb{F}_{2}\right.$ cases.)

be constructed from a reflexive polyhedron ${ }^{1}$. Local mirror symmetry was derived from mirror symmetry for toric Calabi-Yau hypersurfaces by considering a certain limit in the Kähler and complex moduli spaces ${ }^{2}[11,28]$. Chiang-Klemm-Yau-Zaslow [11] gave quite a thorough mathematical treatment to it. Their result can be summarized as follows.

Take a two-dimensional reflexive polyhedron $\Delta$ (see figure 1 for examples). On one side ("lcoal A-model" side), one considers the genus zero local GW invariants of a smooth weak Fano toric surface $\mathbb{P}_{\Sigma\left(\Delta^{*}\right)}$ which is determined by the two-dimensional complete fan $\Sigma\left(\Delta^{*}\right)$ generated by integral points of $\Delta$. On the other side ("local B-model" side), one considers a system of differential equations associated to $\Delta$ called the $A$-hypergeometric system with parameter zero due to Gel'fand-Kapranov-Zelevinsky [16, 17]. Then the statement of local mirror symmetry is that the genus zero local GW invariants can be obtained from solutions of the $A$-hypergeometric system.

Remark 1.1. The problem of computing the local GW invariants, not only at genus zero but also at all genera, is solved completely by the method of the topological vertex [1].

\subsection{Local B-model and the mixed Hodge structure}

When one compares local mirror symmetry with mirror symmetry, it is easy to see an analogy between the A-model (GW invariants) and the local A-model (local GW invariants). To compare the B-model and the local B-model, let us look into them in more detail. A natural framework for

\footnotetext{
${ }^{1}$ See Section 3.5 for the definition of reflexive polyhedra.

${ }^{2}$ This limit typically corresponds to a situation on the A-model side where one considers the effect of a local geometry of a weak Fano surface within a Calabi-Yau threefold. Hence the term "local mirror symmetry". See [28, Section 4].
} 
the B-model is the variation of polarized Hodge structures on $H^{3}\left(X^{\vee}\right)^{3}$ (cf. [12, Ch. 5], [36, Ch. 1, Ch. 3]). One considers

(i) the family $\pi: \mathcal{X} \rightarrow B$ of complex deformations of the Calabi-Yau three-fold $X^{\vee}$,

(ii) a relative holomorphic three form $\Omega_{\mathcal{X} / B}$, which together with the elements obtained by successive applications of the Gauss-Manin connection $\nabla$, spans $H^{3}\left(X^{\vee}\right)$,

(iii) the Picard-Fuchs system for period integrals of $\Omega_{\mathcal{X} / B}$,

(iv) an $\mathcal{O}_{B}$-multilinear symmetric map from $T B \times T B \times T B$ to $\mathcal{O}_{B}$ called the Yukawa coupling

$$
Y_{b}\left(A_{1}, A_{2}, A_{3}\right)=\int_{X_{b}^{\vee}} \nabla_{A_{1}} \nabla_{A_{2}} \nabla_{A_{3}} \Omega_{\mathcal{X} / B} \wedge \Omega_{\mathcal{X} / B}, \quad(b \in B) .
$$

Let us turn to the local B-model. Our proposal in this paper is that a natural language for the local B-model is the mixed Hodge structures and their variations. The mixed Hodge structure (MHS) due to Deligne [13] is a generalization of the Hodge structure with the extra data $\mathcal{W}_{\bullet}$ called the weight filtration. See Section 2. Although the cohomology $H^{*}\left(V^{\circ}\right)$ of an open smooth variety $V^{\circ}$ does not have a Hodge structure in general, it does have a canonical MHS $[13,14]$. There is also a canonical one on the relative cohomology $H^{*}\left(U^{\circ}, V^{\circ}\right)$.

Now, let us explain what are the counterparts of (i)-(iv) in the local Bmodel. Let $\Delta$ be a two-dimensional reflexive polyhedron as above and $F_{a}$ be a $\Delta$-regular Laurent polynomial, i.e., a Laurent polynomial of the form

$$
F_{a}\left(t_{1}, t_{2}\right)=\sum_{m \in \Delta \cap \mathbb{Z}^{2}} a_{m} t^{m} \in \mathbb{C}\left[t_{1}^{ \pm 1}, t_{2}^{ \pm 1}\right]
$$

which satisfies a certain regularity condition (cf. Definition 3.1). In the literature, two closely related manifolds associated to $F_{a}$ are considered: the one is the affine curve $C_{a}^{\circ}$ in the two-dimensional algebraic torus $\mathbb{T}^{2}=\left(\mathbb{C}^{*}\right)^{2}$ defined by $F_{a}\left(t_{1}, t_{2}\right)=0[11$, Section 6$]$, and the other is the open threefold $Z_{a}^{\circ} \subset \mathbb{T}^{2} \times \mathbb{C}^{2}$ defined by $F_{a}+x y=0[23$, Section 8$]$. As we shall see, they give the same result. By varying the parameter $a=\left(a_{m}\right)$, we have a family of affine curves $\mathcal{Z} \rightarrow \mathbb{L}_{\text {reg }}(\Delta)$ and a family of open threefolds $\mathcal{Z}^{\prime} \rightarrow \mathbb{L}_{\text {reg }}(\Delta)$.

\footnotetext{
${ }^{3}$ Throughout the paper, the coefficient of the cohomology group is $\mathbb{C}$ unless otherwise specified.
} 
By taking a quotient by the following action of $\mathbb{T}^{3}=\left(\mathbb{C}^{*}\right)^{3}$,

$$
F_{a}\left(t_{1}, t_{2}\right) \mapsto \lambda_{0} F_{a}\left(\lambda_{1} t_{1}, \lambda_{2} t_{2}\right), \quad\left(\lambda_{0}, \lambda_{1}, \lambda_{2}\right) \in \mathbb{T}^{3},
$$

we also have the quotient families $\mathcal{Z} / \mathbb{T}^{3} \rightarrow \mathcal{M}(\Delta)$ and $\mathcal{Z}^{\prime} / \mathbb{T}^{3} \rightarrow \mathcal{M}(\Delta)$. These correspond to (i). As a counterpart of (ii), we consider, for the affine curve $C_{a}^{\circ}$, the class

$$
\omega_{0}=\left[\left(\frac{d t_{1}}{t_{1}} \wedge \frac{d t_{2}}{t_{2}}, 0\right)\right] \in H^{2}\left(\mathbb{T}^{2}, C_{a}^{\circ}\right)^{4},
$$

in the relative cohomology $H^{2}\left(\mathbb{T}^{2}, C_{a}^{\circ}\right)$, and for the open threefold $Z_{a}^{\circ}$, the class of a holomorphic 3-form:

$$
\omega_{a}=\left[\operatorname{Res} \frac{1}{F_{a}+x y} \frac{d t_{1}}{t_{1}} \frac{d t_{2}}{t_{2}} d x d y\right] \in H^{3}\left(Z_{a}^{\circ}\right) .
$$

The counterpart of (iii) is the $A$-hypergeometric system as explained in [11]. Batyrev [5] and Stienstra [33] studied the variation of MHS (VMHS) on $H^{2}\left(\mathbb{T}^{2}, C_{a}^{\circ}\right)$ and showed the followings: $H^{2}\left(\mathbb{T}^{2}, C_{a}^{\circ}\right) \cong \mathbb{C} \omega_{0} \oplus P H^{1}\left(C_{a}^{\circ}\right)$ is isomorphic to a certain vector space $\mathcal{R}_{F_{a}} ; \omega_{0}$ and elements obtained by successive applications of the Gauss-Manin connection span $H^{2}\left(\mathbb{T}^{2}, C_{a}^{\circ}\right) ; \omega_{0}$ satisfies the $A$-hypergeometric system considered in [11]. For the polyhedron \#1 in figure 1, Takahashi [34] independently showed that integrals

$$
\int_{\Gamma} \omega_{0}, \quad \Gamma \in H_{2}\left(\mathbb{T}^{2}, C_{a}^{\circ}, \mathbb{Z}\right),
$$

over 2-chains $\Gamma$ whose boundaries lie in $C_{a}^{\circ}$ satisfy the same differential equation. For the open threefold $Z_{a}^{\circ}$, there is a result by Hosono [25] that integrals

$$
\int_{\gamma} \omega_{a}, \quad \gamma \in H_{3}\left(Z_{a}^{\circ}, \mathbb{Z}\right),
$$

satisfy exactly the same $A$-hypergeometric system. It has been known that $H^{3}\left(Z_{a}^{\circ}\right) \cong H^{2}\left(\mathbb{T}^{2}, C_{a}^{\circ}\right)$. Gross [20, Section 4] described the isomorphism and mentioned that the integration of $\omega_{a}$ over a 3-cycle reduces to that of $\omega_{0}$ over a 2-chain under the isomorphism. In this paper, we shall study the (V)MHS of $H^{3}\left(Z_{a}^{\circ}\right)$ and show that it has the same description as $H^{2}\left(\mathbb{T}^{2}, C_{a}^{\circ}\right) \cong \mathcal{R}_{F_{a}}$ and that $\omega_{a}$ plays the same role as $\omega_{0}$. This is one of the main results of this paper (cf. Theorem 5.1).

\footnotetext{
${ }^{4}$ Note that the class $\omega_{0}$ depends on the parameter $a$, although it is not indicated in the notation.
} 
Remark 1.2. In [11], Chiang et al. considered the "1-form" $\operatorname{Res}_{F_{a}=0}\left(\log F_{a}\right)$ $\omega_{0}$ on $C_{a}^{\circ}$ and argued that its period integrals satisfy the $A$-hypergeometric system. The result by Batyrev, Stienstra, and Takahashi implies that $\omega_{0} \in$ $H^{2}\left(\mathbb{T}^{2}, C_{a}^{\circ}\right)$ gives a rigorous definition of this "1-form". This point was mentioned in [20].

Remark 1.3. Calculation of the (V)MHS of $H^{3}\left(Z_{a}^{\circ}\right)$ in this paper closely follows the result by Batyrev on the MHS of affine hypersurfaces in algebraic tori [5].

\subsection{Weight filtration and the Yukawa coupling}

At this point, one may ask what is the role of the weight filtration. Our answer is that it is needed to define an analogue of the Yukawa coupling. It is the main motivation of the present work. In general, the lowest level subspace of the weight filtration in $H^{*}\left(V^{\circ}\right)$ is the image of the cohomology $H^{*}(V)$ of a smooth compactification $V$ (see, e.g., [32, Proposition 6.30]). In our cases, it turns out that the lowest level subspace $\mathcal{W}_{1} H^{2}\left(\mathbb{T}^{2}, C_{a}^{\circ}\right.$ ) (resp. $\mathcal{W}_{3} H^{3}\left(Z_{a}^{\circ}\right)$ ) of the weight filtration on $H^{2}\left(\mathbb{T}^{2}, C_{a}^{\circ}\right)$ (resp. $H^{3}\left(Z^{\circ}\right)$ ) is isomorphic to $H^{1}\left(C_{a}\right)$ (resp. $H^{3}\left(Z_{a}\right)$ ), where $C_{a}$ (resp. $Z_{a}$ ) is a smooth compactification of $C_{a}^{\circ}$ (resp. $Z_{a}^{\circ}$ ). Thus we can use the intersection product on $H^{1}\left(C_{a}\right)$ or $H^{3}\left(Z_{a}\right)$ to define an analogue of the Yukawa coupling.

As a counterpart to (iv), we propose the following definition (Definition 6.1). Consider the family of affine curves $\mathcal{Z} \rightarrow \mathbb{L}_{\text {reg }}(\Delta)$. Let $T^{0} \mathbb{L}_{\text {reg }}(\Delta)$ be the subbundle of the holomorphic tangent bundle $T \mathbb{L}_{\mathrm{reg}}(\Delta)$ spanned by $\partial_{a_{0}}{ }^{5}$. Our Yukawa coupling is a multilinear map from $T \mathbb{L}_{\mathrm{reg}}(\Delta) \times T \mathbb{L}_{\mathrm{reg}}(\Delta)$ $\times T^{0} \mathbb{L}_{\text {reg }}(\Delta)$ to $\mathcal{O}_{\mathbb{L}_{\text {reg }}(\Delta)}$. Take three vector fields $\left(A_{1}, A_{2}, A_{3}\right) \in T \mathbb{L}_{\text {reg }}(\Delta) \times$ $T \mathbb{L}_{\text {reg }}(\Delta) \times T^{0} \mathbb{L}_{\text {reg }}(\Delta)$. By the result on VMHS, we see that $\nabla_{A_{3}} \omega_{0}$ can be regarded as a (1,0)-form on $C_{a}$, and that although $\nabla_{A_{1}} \nabla_{A_{2}} \omega_{0}$ may not be in $\mathcal{W}_{1}$, we can associate a 1 -form $\left(\nabla_{A_{1}} \nabla_{A_{2}} \omega_{0}\right)^{\prime}$ on $C_{a}$ (Lemma 6.1). We define

$$
\operatorname{Yuk}\left(A_{1}, A_{2} ; A_{3}\right)=\sqrt{-1} \int_{C_{a}}\left(\nabla_{A_{1}} \nabla_{A_{2}} \omega_{0}\right)^{\prime} \wedge \nabla_{A_{3}} \omega_{0} .
$$

It is also possible to define the Yukawa coupling using the family of open threefolds. In fact, they are the same up to multiplication by a nonzero constant. We also have a similar definition for the quotient family (Section 6.5).

In addition to the above geometric definition, we give an algebraic description of the Yukawa coupling via a certain pairing considered by Batyrev [5]

\footnotetext{
${ }^{5}$ Here $a_{0}$ is the parameter corresponds to the origin $(0,0) \in \Delta \cap \mathbb{Z}^{2}$.
} 
(cf. Sections 6.2, 6.3). We also derive the differential equations for them (Proposition 6.2, Lemma 6.2). These results enables us to compute the Yukawa couplings at least in the examples shown in figure 1 (cf. Example 6.13, Section 8). They agree with the known results $[2,3,8,15,22,29]$. We also see that they are mapped to the local A-model Yukawa couplings by the mirror maps (cf. Example 6.15, Section 8).

\subsection{Local B-model at higher genera}

If we are to pursue further the analogy between mirror symmetry and local mirror symmetry to higher genera, the first thing to do is to formulate an analogue of the so-called special Kähler geometry. It is a Kähler metric on the moduli $B$ of complex deformations of a Calabi-Yau threefold $X^{\vee}$ whose curvature satisfies a certain equation called the special geometry relation. In the setting of the local B-model, we define a Hermitian metric on the rank one subbundle $T^{0} \mathcal{M}(\Delta)$ of $T \mathcal{M}(\Delta)$ and derive an equation similar to the special geometry relation (Lemma 7.1).

Next, we consider Bershadsky-Cecotti-Ooguri-Vafa's (BCOV's) holomorphic anomaly equation [7]. It is a partial differential equation for the B-model topological string amplitudes $F_{g}{ }^{\prime}{ }^{6}$ which involves the Kähler metric, its Kähler potential and the Yukawa coupling. By making use of the result on the VMHS of $H^{2}\left(\mathbb{T}^{2}, C_{a}^{\circ}\right)$ (or $H^{3}\left(Z_{a}^{\circ}\right)$ ), we propose how to adapt the holomorphic anomaly equation to the setting of the local B-model (equations (7.4), (7.5)). We also explain it from Witten's geometric quantization approach [39].

In the examples shown in figure 1 , we checked that the solutions of this holomorphic anomaly equation with appropriate holomorphic ambiguities and with the holomorphic limit give the correct local GW invariants for $g=1,2$ at least for small degrees.

Remark 1.4. It is known that, in the local setting, the Kähler potential drops out from BCOV's holomorphic anomaly equation, and consequently the equation is solved by a certain Feynman rule with only one type of propagators $S^{i, j}$ with two indices $[2,3,22,24,29]$. Moreover, it is also known that essentially only one direction in $\mathcal{M}(\Delta)$ corresponding to the moduli of the elliptic curve $C_{a}$ is relevant. Our description of BCOV's holomorphic anomaly equation is based on these results.

\footnotetext{
${ }^{6}$ Its mathematical definition is yet unknown for $g \geq 2$.
} 


\subsection{Plan of the paper}

In Section 2, we recall the definition of the MHS. In Section 3, we define the vector space $\mathcal{R}_{F_{a}}$ following Batyrev [5] and recall Gel'fand-KapranovZelevinsky's $A$-hypergeometric system [16,17]. In Section 4, we give descriptions due to Batyrev [5] and Stienstra [33] of (V)MHS on the relative cohomology $H^{n}\left(\mathbb{T}^{n}, V_{a}^{\circ}\right)$ of the pair of the $n$-dimensional algebraic torus $\mathbb{T}^{n}$ and an affine hypersurface $V_{a}^{\circ}$ in terms of $\mathcal{R}_{F_{a}}$ (Theorem 4.2). In Section 5, we state the result on the MHS on the cohomology $H^{3}\left(Z_{a}^{\circ}\right)$ of the open threefold $Z_{a}^{\circ}$ and its relationship to that on $H^{2}\left(\mathbb{T}^{2}, C_{a}^{\circ}\right)$ (Theorem 5.1). The details for the calculation of $H^{3}\left(Z_{a}^{\circ}\right)$ are given in Section A. In Section 6, we define the Yukawa coupling and study its properties. In Section 7, we propose a holomorphic anomaly equation for the local B-model.

We note that polyhedra dealt with in Sections 3 and 4 are convex integral polyhedra, while in Sections 5, 6 and 7, only two-dimensional reflexive polyhedra are considered.

The examples treated in this article are listed in figure 1 . These will be sometimes called the cases of $\mathbb{P}^{2}, \mathbb{F}_{0}, \mathbb{F}_{1}, \mathbb{F}_{2}$ according to their local A-model toric surfaces. The $\mathbb{P}^{2}$ case appears in the course of the paper. The others are summarized in Section 8.

\subsection{Notations}

Throughout the paper, we use the following notations. $\mathbb{T}^{n}$ denotes the $n$ dimensional algebraic torus $\left(\mathbb{C}^{*}\right)^{n}$. For $m=\left(m_{1}, \ldots, m_{n}\right) \in \mathbb{Z}^{n}, t^{m}$ stands for the Laurent monomial $t_{1}^{m_{1}} \cdots t_{n}^{m_{n}} \in \mathbb{C}\left[t_{1}^{ \pm 1}, \ldots, t_{n}^{ \pm 1}\right]$. For a variable $x, \theta_{x}$ is the logarithmic derivative $x \partial_{x}$.

\section{Preliminaries on the mixed Hodge structures}

In this section, we recall the mixed Hodge structure of Deligne [13,14]. See also $[32,37,38]$.

A mixed Hodge structure (MHS) $H$ is the triple $H=\left(H_{\mathbb{Z}}, \mathcal{W}_{\bullet}, \mathcal{F}^{\bullet}\right)$, where $H_{\mathbb{Z}}$ is a finitely generated abelian group, $\mathcal{W}_{\bullet}$ is an increasing filtration (called the weight filtration) on $H_{\mathbb{Q}}=H_{\mathbb{Z}} \otimes \mathbb{Q}$, and $\mathcal{F}^{\bullet}$ is a decreasing filtration (called the Hodge filtration) on $H_{\mathbb{C}}=H_{\mathbb{Z}} \otimes \mathbb{C}$ such that for each graded quotient

$$
\operatorname{Gr}_{n}^{\mathcal{W}}\left(H_{\mathbb{Q}}\right):=\mathcal{W}_{n} / \mathcal{W}_{n-1},
$$


with respect to the weight filtration $\mathcal{W}_{\bullet}$, the Hodge filtration $\mathcal{F}^{\bullet}$ induces a decomposition

$$
\operatorname{Gr}_{n}^{\mathcal{W}}\left(H_{\mathbb{C}}\right)=\bigoplus_{p+q=n} H^{p, q}
$$

with $\overline{H^{p, q}}=H^{q, p}$, where $H^{p, q}:=\operatorname{Gr}_{\mathcal{F}}^{p} \operatorname{Gr}_{p+q}^{\mathcal{W}}\left(H_{\mathbb{C}}\right)$ and the bar denotes the complex conjugation. We say that the weight $m \in \mathbb{Z}$ occurs in $H$ if $\mathrm{Gr}_{m}^{\mathcal{W}} \neq 0$, and that $H$ is a pure Hodge structure of weight $m$ if $m$ is the only weight which occurs in $H$. By the classical Hodge theory, if $X$ is a compact Kähler manifold, then $H^{k}(X)$ carries a pure Hodge structure of weight $k$.

Let $V^{\circ}$ be a smooth open algebraic variety of dimension $n$. By Deligne $[13,14]$, there is a canonical MHS on $H^{k}\left(V^{\circ}\right)$. The weights of $H^{k}\left(V^{\circ}\right)$ may occur in the range $[k, 2 k]$ if $k \leq n$ and in $[k, 2 n]$ if $k \geq n$. The construction goes as follows. Take a smooth compactification $V$ of $V^{\circ}$ such that the divisor $D=V \backslash V^{\circ}$ is a simple normal crossing divisor, and consider meromorphic differential forms on $V$ which may have logarithmic poles along the divisor $D$. Then the Hodge filtration is given by the degree of logarithmic forms while the weight filtration is given by the pole order. The constructed MHS does not depend on the chosen compactification $V$ and is functorial, i.e., any morphism $f: V^{\circ} \rightarrow U^{\circ}$ of varieties induces a morphism $f^{*}: H^{*}\left(U^{\circ}\right) \rightarrow H^{*}\left(V^{\circ}\right)$ of MHS's.

Let $\iota: V^{\circ} \hookrightarrow U^{\circ}$ be an immersion between two smooth open algebraic varieties. By [14], there exists a canonical MHS on the relative cohomology $H^{k}\left(U^{\circ}, V^{\circ}\right)$. The construction is similar to the one above (cf. [32, Ch. 5], [38, Ch. 8]). The long exact sequence

$$
\cdots \stackrel{\iota^{*}}{\longrightarrow} H^{k-1}\left(V^{\circ}\right) \longrightarrow H^{k}\left(U^{\circ}, V^{\circ}\right) \longrightarrow H^{k}\left(U^{\circ}\right) \stackrel{\iota^{*}}{\longrightarrow} H^{k}\left(V^{\circ}\right) \longrightarrow \cdots,
$$

is an exact sequence of MHS's. The weights of $H^{k}\left(U^{\circ}, V^{\circ}\right)$ may occur in $[k-1,2 k]$.

The $m$ th Tate structure $T(m)$ is the pure Hodge structure of weight $-2 m$ on the lattice $(2 \pi \sqrt{-1})^{m} \mathbb{Z} \subset \mathbb{C}$ which is of type $(-m,-m)$, i.e., $T(m)_{\mathbb{C}}=$ $T(m)^{-m,-m}$.

Example 2.1. The MHS on $H^{m}\left(\mathbb{T}^{n}\right)$ is $T(-m)^{\oplus}\left(\begin{array}{c}n \\ m\end{array}\right)$ for $0 \leq m \leq n$. See [5, Example 3.9].

Example 2.2. Let $C$ be a smooth projective curve and $C^{\circ}=C \backslash D$ be an affine curve, where $D=\left\{p_{1}, \ldots, p_{m}\right\}$ is a set of distinct $m$-points on $C$. We 
describe the MHS on $H^{1}\left(C^{\circ}\right)$. The weight filtration $\mathcal{W}_{\bullet}$ and the Hodge filtration $\mathcal{F}^{\bullet}$ are

$$
\begin{aligned}
& 0 \subset \mathcal{W}_{1}=H^{1}(C) \subset \mathcal{W}_{2}=H^{1}\left(C^{\circ}\right), \\
& 0 \subset \mathcal{F}^{1}=H^{0}\left(\Omega_{C}^{1}(\log D)\right) \subset \mathcal{F}^{0}=H^{1}\left(C^{\circ}\right),
\end{aligned}
$$

where $\Omega_{C}^{1}(\log D)$ is the sheaf of logarithmic 1 -forms on $(C, D)$. The "Hodge decomposition" of $\operatorname{Gr}_{1}^{\mathcal{W}}\left(H^{1}\left(C^{\circ}\right)\right)=H^{1,0} \oplus H^{0,1}$ is the same as that of $H^{1}(C)$ :

$$
H^{1,0}=H^{1,0}(C), \quad H^{0,1}=H^{0,1}(C) .
$$

That of $\operatorname{Gr}_{2}^{\mathcal{W}}\left(H^{1}\left(C^{\circ}\right)\right)=H^{2,0} \oplus H^{1,1} \oplus H^{0,2}$ is

$$
H^{1,1}=H^{0}\left(\Omega_{C}^{1}(\log D)\right) / H^{0}\left(\Omega_{C}^{1}\right), \quad H^{2.0}=H^{0,2}=0 .
$$

Hence, the "Hodge numbers" of $H^{1}\left(C^{\circ}\right)$ are

$$
\begin{array}{r|rrr} 
& p=0 & 1 & 2 \\
\hline q=0 & & g & 0 \\
1 & g & m-1 & \\
2 & 0 & &
\end{array},
$$

where $g$ is the genus of $C$. The MHS on $H^{1}\left(C^{\circ}\right)$ is an extension of $T(-1)^{\oplus(m-1)}$ by $H^{1}(C)$ in the sense of [10]:

$$
0 \rightarrow H^{1}(C) \rightarrow H^{1}\left(C^{\circ}\right) \rightarrow T(-1)^{\oplus(m-1)} \rightarrow 0 .
$$

\section{Polyhedron, Jacobian $\operatorname{ring}, \mathcal{R}_{F}$ and $A$-hypergeometric system}

A convex integral polyhedron $\Delta \subset \mathbb{R}^{n}$ is the convex hull of some finite set in $\mathbb{Z}^{n}$. The set of integral points in $\Delta$ is denoted by $A(\Delta)$ and its cardinality by $l(\Delta):=\# A(\Delta)$.

\section{$3.1 \Delta$-regularity}

Let $\Delta$ be an $n$-dimensional integral convex polyhedron. Equip the ring $\mathbb{C}\left[t_{0}, t_{1}^{ \pm 1}, \ldots, t_{n}^{ \pm 1}\right]$ with the grading given by $\operatorname{det} t_{0}^{k} t^{m}=k$. Define $\mathbf{S}_{\Delta}$ to be 
its subring

$$
\mathbf{S}_{\Delta}=\bigoplus_{k \geq 0} \mathbf{S}_{\Delta}^{k}, \quad \mathbf{S}_{\Delta}^{k}=\bigoplus_{m \in \Delta(k)} \mathbb{C} t_{0}^{k} t^{m}
$$

where

$$
\Delta(k):=\left\{m \in \mathbb{R}^{n} \mid \frac{m}{k} \in \Delta\right\}(k \geq 1), \quad \Delta(0):=\{0\} \subset \mathbb{R}^{n} .
$$

Recall that the Newton polyhedron of a Laurent polynomial

$$
F=\sum_{m} a_{m} t^{m} \in \mathbb{C}\left[t_{1}^{ \pm 1}, \ldots, t_{n}^{ \pm 1}\right]
$$

is the convex hull of $\left\{m \in \mathbb{Z}^{n} \mid a_{m} \neq 0\right\}$ in $\mathbb{R}^{n}$. Denote by $\mathbb{L}(\Delta)$ the space of Laurent polynomials whose Newton polyhedra are $\Delta$.

Definition 3.1. A Laurent polynomial $F$ is said to be $\Delta$-regular if $F \in \mathbb{L}(\Delta)$ and, for every $l$-dimensional face $\Delta^{\prime} \subset \Delta(0<l \leq n)$, the equations

$$
F^{\Delta^{\prime}}:=\sum_{m \in \Delta^{\prime} \cap \mathbb{Z}^{n}} a_{m} t^{m}=0, \quad \frac{\partial F^{\Delta^{\prime}}}{\partial t_{1}}=0, \ldots, \frac{\partial F^{\Delta^{\prime}}}{\partial t_{n}}=0,
$$

have no common solutions in $\mathbb{T}^{n}$. Denote by $\mathbb{L}_{\text {reg }}(\Delta)$ the space of $\Delta$-regular Laurent polynomials.

Example 3.1. Let $\Delta \subset \mathbb{R}^{2}$ be the polyhedron $\# 1$ in figure 1, which is the convex hull of $\{(1,0),(0,1),(-1,-1)\}$. Let $F \in \mathbb{L}(\Delta)$ which is of the form

$$
F=a_{1} t_{1}+a_{2} t_{2}+\frac{a_{3}}{t_{1} t_{2}}+a_{0}, \quad\left(a_{0}, a_{1}, a_{2}, a_{3} \in \mathbb{C}\right) .
$$

We wrote $a_{(1,0)}=a_{1}, a_{(0,1)}=a_{2}, a_{(-1,-1)}=a_{3}$ for simplicity. Then we have

$$
F \in \mathbb{L}_{\mathrm{reg}}(\Delta) \Longleftrightarrow \frac{a_{0}^{3}}{a_{1} a_{2} a_{3}}+27 \neq 0, a_{1} a_{2} a_{3} \neq 0 .
$$

\subsection{Jacobian ring, $\mathcal{R}_{F}$ and filtrations}

For $F \in \mathbb{L}(\Delta)$, let $\mathcal{D}_{i}(i=0, \ldots, n)$ be the following differential operators acting on $\mathbf{S}_{\Delta}$ :

$$
\mathcal{D}_{0}:=\theta_{t_{0}}+t_{0} F, \quad \mathcal{D}_{i}:=\theta_{t_{i}}+t_{0} \theta_{t_{i}} F, \quad(i=1, \ldots, n) .
$$


Definition 3.2. Define $\mathbb{C}$-vector spaces $\mathcal{R}_{F}$ and $\mathcal{R}_{F}^{+}$by

$$
\mathcal{R}_{F}:=\mathbf{S}_{\Delta} / \sum_{i=0}^{n} \mathcal{D}_{i} \mathbf{S}_{\Delta}, \quad \mathcal{R}_{F}^{+}:=\mathbf{S}_{\Delta}^{+} / \sum_{i=0}^{n} \mathcal{D}_{i} \mathbf{S}_{\Delta},
$$

where $\mathbf{S}_{\Delta}^{+}=\sum_{k \geq 1} \mathbf{S}_{\Delta}^{k}$

Obviously, $\mathcal{R}_{F}=\mathcal{R}_{F}^{+} \oplus \mathbf{S}_{\Delta}^{0}$.

We consider two filtrations on the vector spaces $\mathcal{R}_{F}$. The $\mathcal{E}$-filtration on $\mathbf{S}_{\Delta}$ is a decreasing filtration

$$
\mathcal{E}: \cdots \supset \cdots \supset \mathcal{E}^{-k} \supset \cdots \mathcal{E}^{-1} \supset \mathcal{E}^{0} \supset \cdots,
$$

where $\mathcal{E}^{-k}$ is the subspace spanned by all monomials of the degree $\leq k$. This induces filtrations on $\mathcal{R}_{F}$ and $\mathcal{R}_{F}^{+}$which are denoted by $\mathcal{E}$ and $\mathcal{E}_{+}$, respectively. It holds that $\mathcal{E}^{-n}=\mathcal{R}_{F}$.

Definition 3.3. Let $J_{F}$ be the ideal in $\mathbf{S}(\Delta)$ generated by $t_{0} F, t_{0} \theta_{t_{1}} F, \ldots, t_{0}$ $\theta_{t_{n}} F$. The Jacobian ring $R_{F}$ is defined as $\mathbf{S}_{\Delta} / J_{F}$. Denote by $R_{F}^{i}$ the $i$ th homogeneous piece of $R_{F}$.

The graded quotient of $\mathcal{R}_{F}$ with respect to the $\mathcal{E}$-filtration is given by the Jacobian ring

$$
\mathrm{Gr}_{\mathcal{E}}^{-i} \mathcal{R}_{F}=R_{F}^{i}
$$

Denote by $I_{\Delta}^{(j)}(0 \leq j \leq n+1)$ the homogeneous ideals in $\mathbf{S}_{\Delta}$ generated as $\mathbb{C}$-subspaces by all monomials $t_{0}^{k} t^{m}$, where $k \geq 1$ and $m \in \Delta(k)$ which does not belong to any face of codimension $j$. We set $I_{\Delta}^{(n+2)}=\mathbf{S}_{\Delta}$. These form an increasing chain of ideals in $\mathbf{S}_{\Delta}$

$$
0=I_{\Delta}^{(0)} \subset I_{\Delta}^{(1)} \subset I_{\Delta}^{(2)} \subset \cdots \subset I_{\Delta}^{(n+1)}=\mathbf{S}_{\Delta}^{+} \subset I_{\Delta}^{(n+2)}=\mathbf{S}_{\Delta} .
$$

Let $\mathcal{I}_{j} \subset \mathcal{R}_{F}$ be the image of $I_{\Delta}^{(j)}$. These subspaces define an increasing filtration $\mathcal{I}$ on $\mathcal{R}_{F}$ :

$$
0=\mathcal{I}_{0} \subset \mathcal{I}_{1} \subset \mathcal{I}_{2} \subset \cdots \subset \mathcal{I}_{n+1}=\mathcal{R}_{F}^{+} \subset \mathcal{I}_{n+2}=\mathcal{R}_{F} .
$$

Later we will see that $\mathcal{R}_{F}$ is isomorphic to the cohomology mentioned in Section 1.2. The $\mathcal{I}$-(resp. $\mathcal{E}$-)filtration describes the weight (resp. Hodge) filtration of the MHS on it. 
Example 3.2. Let $\Delta$ be the polyhedron $\# 1$ in figure 1 . Assume that $F \in \mathbb{L}_{\text {reg }}(\Delta)$. Then we have

$$
\mathcal{R}_{F} \cong \mathbb{C} 1 \oplus \mathbb{C} t_{0} \oplus \mathbb{C} t_{0}^{2}
$$

The $\mathcal{I}$ - and the $\mathcal{E}$-filtrations are

$$
\begin{aligned}
& \mathcal{I}_{3}=\mathcal{I}_{2}=\mathcal{I}_{1} \cong \mathbb{C} t_{0} \oplus \mathbb{C} t_{0}^{2}, \quad \mathcal{I}_{4}=\mathcal{R}_{F} . \\
& \mathcal{E}^{0}=\mathbb{C} 1, \quad \mathcal{E}^{-1}=\mathbb{C} 1 \oplus \mathbb{C} t_{0}, \quad \mathcal{E}^{-2}=\mathcal{R}_{F} .
\end{aligned}
$$

\subsection{Derivations with respect to parameters}

Let $a=\left(a_{m}\right)_{m \in A(\Delta)}$ be algebraically independent coefficients. Consider the $\mathbb{C}[a]$-module $\mathbf{S}_{\Delta}[a]:=\mathbf{S}_{\Delta} \otimes \mathbb{C}[a]$. Let

$$
\mathcal{R}_{F}[a]:=\mathbf{S}_{\Delta}[a] /\left(\sum_{i=0}^{n} \mathcal{D}_{i} \mathbf{S}_{\Delta}[a]\right) .
$$

Define the action of differential operators $\mathcal{D}_{a_{m}}(m \in A(\Delta))$ on $\mathbf{S}_{\Delta}[a]$ by

$$
\mathcal{D}_{a_{m}}=\frac{\partial}{\partial a_{m}}+t_{0} t^{m}
$$

Since this action commutes with that of $\mathcal{D}_{i}(i=0,1, \ldots, n)$, it induces an action of $\mathcal{D}_{a_{m}}$ on $\mathcal{R}_{F}[a]$.

We shall see that the operator $\mathcal{D}_{a_{m}}$ corresponds to the Gauss-Manin connection $\nabla_{a_{m}}$ on the cohomology of our interest (cf. Sections 4.3, 5). Note that $\mathcal{D}_{a_{m}}$ preserves the $\mathcal{I}$-filtration: $\mathcal{D}_{a_{m}} \mathcal{I}^{j} \subset \mathcal{I}^{j}$. This corresponds to the fact that the weight filtration is preserved by the VMHS's. Note also that $\mathcal{D}_{a_{m}}$ decreases $\mathcal{E}$-filtration by one: $\mathcal{D}_{a_{m}} \mathcal{E}^{-k} \subset \mathcal{E}^{-k-1}$. This corresponds to the Griffiths transversality [35].

\section{$3.4 \quad A$-hypergeometric system}

We briefly recall the $A$-hypergeometric system of Gel'fand-KapranovZelevinsky $[16,17]$ in a form suitable to our situation. Let $\Delta$ be an $n$ dimensional integral convex polyhedron. For $\Delta$, the lattice of relations is 
defined by

$$
L(\Delta):=\left\{l=\left(l_{m}\right)_{m \in A(\Delta)} \in \mathbb{Z}^{l(\Delta)} \mid \sum_{m \in A(\Delta)} l_{m} m=0, \sum_{m \in A(\Delta)} l_{m}=0\right\} .
$$

The $A$-hypergeometric system associated to $\Delta$ (with parameters $(0, \ldots, 0) \in$ $\left.\mathbb{C}^{n+1}\right)$ is the following system of linear differential equations for $\Phi(a)$ :

$$
\mathcal{T}_{i} \Phi(a)=0(i=0,1, \ldots, n), \quad \square_{l} \Phi(a)=0(l \in L(\Delta))
$$

where

$$
\begin{aligned}
\mathcal{T}_{0} & =\sum_{m \in A(\Delta)} \theta_{a_{m}}, \quad \mathcal{T}_{i}=\sum_{m \in A(\Delta)} m_{i} \theta_{a_{m}} \quad(1 \leq i \leq n), \\
\square_{l} & =\prod_{\substack{m \in A(\Delta) ; \\
l_{m}>0}} \partial_{a_{m}}^{l_{m}}-\prod_{\substack{m \in A(\Delta) ; \\
l_{m}<0}} \partial_{a_{m}}^{-l_{m}} .
\end{aligned}
$$

The number of independent solutions is equal to the volume ${ }^{7}$ of the polyhedron $\Delta[16]$.

Example 3.3. In the case when $\Delta$ is the polyhedron $\# 1$ in figure 1 , the lattice of relations $L(\Delta)$ has rank one and generated by $(-3,1,1,1)$. For simplicity we write $a_{(1,0)}=a_{1}, a_{(0,1)}=a_{2}, a_{(-1,-1)}=a_{3}$. The $A$-hypergeometric system is

$$
\begin{aligned}
& \left(\theta_{a_{1}}-\theta_{a_{3}}\right) \Phi(a)=0, \quad\left(\theta_{a_{2}}-\theta_{a_{3}}\right) \Phi(a)=0 \\
& \left(\theta_{a_{0}}+\theta_{a_{1}}+\theta_{a_{2}}+\theta_{a_{3}}\right) \Phi(a)=0 \\
& \left(\partial_{a_{1}} \partial_{a_{2}} \partial_{a_{3}}-\partial_{a_{0}}^{3}\right) \Phi(a)=0 .
\end{aligned}
$$

It is equivalent to $\Phi(a)=f(z), z=\frac{a_{1} a_{2} a_{3}}{a_{0}^{3}}$ and

$$
\left[\theta_{z}^{3}+3 z \theta_{z}\left(3 \theta_{z}+1\right)\left(3 \theta_{z}+2\right)\right] f(z)=0
$$

Solutions about $z=0$ are obtained in [11, equation $(6.22)]$ :

$$
\begin{aligned}
& \varpi(z ; 0)=1, \quad t:=\left.\partial_{\rho} \varpi(z ; \rho)\right|_{\rho=0}=\log z+3 H(z), \\
& \partial_{S} F=\left.\frac{1}{2 !} \partial_{\rho}^{2} \varpi(z ; \rho)\right|_{\rho=0}=\frac{1}{2}(\log z)^{2}+\cdots,
\end{aligned}
$$

\footnotetext{
${ }^{7}$ Here the volume is normalized so that the fundamental simplex in $\mathbb{R}^{n}$ has volume one.
} 
where

$$
\varpi(z ; \rho)=\sum_{n \geq 0} \frac{(3 \rho)_{3 n}}{(1+\rho)_{n}^{3}}(-1)^{n} z^{n+\rho}, \quad H(z)=\sum_{n \geq 1} \frac{(3 n-1) !}{n !^{3}}(-z)^{n} .
$$

Here $(\alpha)_{n}$ denotes the Pochhammer symbol: $(\alpha)_{n}=(\alpha)(\alpha+1) \cdots(\alpha+n-$ 1) for $n \geq 1,(\alpha)_{n}=1$ for $n=0$.

Proposition 3.1. 1. For each $F \in \mathbb{L}_{\mathrm{reg}}(\Delta), \mathcal{R}_{F}$ is spanned by $\mathcal{D}_{a_{m_{1}}} \ldots$ $\mathcal{D}_{a_{m_{k}}} 1\left(0 \leq k \leq n, m_{1}, \ldots, m_{k} \in A(\Delta)\right)$.

2. In $\mathcal{R}_{F}[a]$, the element 1 satisfies the A-hypergeometric system (3.8) with $\partial_{a_{m}}(m \in A(\Delta))$ replaced by $\mathcal{D}_{a_{m}}$.

Proof. 1. This follows because $\mathbf{S}_{\Delta}$ is spanned by monomials obtained by successive applications of $\mathcal{D}_{a_{m}}$ to 1 and because $\mathcal{R}_{F}=\mathcal{E}^{-n}$.

2. In the ring $\mathbf{S}_{\Delta}[a]$, it holds that

$$
\begin{aligned}
& \left(\left.\mathcal{T}_{i}\right|_{\theta_{a_{m} \rightarrow a_{m}} \mathcal{D}_{a_{m}}}\right) 1=\mathcal{D}_{i} 1 \quad(0 \leq i \leq n), \\
& \left(\left.\square_{l}\right|_{a_{a_{m}} \rightarrow \mathcal{D}_{a_{m}}}\right) 1=\prod_{m: l_{m}>0}\left(t^{m}\right)^{l_{m}}-\prod_{m: l_{m}<0}\left(t^{m}\right)^{-l_{m}}=0 .
\end{aligned}
$$

\subsection{Reflexive polyhedra}

Recall from [6, Section 4] the following:

Definition 3.4. An $n$-dimensional convex integral polyhedron $\Delta \subset \mathbb{R}^{n}$ is reflexive if $0 \in \Delta$ and the distance between 0 and the hypersurface generated by each codimension-one face $\Delta^{\prime}$ is equal to 1 , i.e., for each codimension-one face $\Delta^{\prime}$ of $\Delta$, there exists an integral primitive vector $v_{\Delta^{\prime}} \in \mathbb{Z}^{n}$ such that $\Delta^{\prime}=\left\{m \in \Delta \mid\left\langle v_{\Delta^{\prime}}, m\right\rangle=1\right\}$.

In the case when $n=2$, it is known that there are exactly sixteen twodimensional reflexive polyhedra (see [11, figure 1]). Let $\Delta$ be a twodimensional reflexive polyhedron and $F \in \mathbb{L}_{\mathrm{reg}}(\Delta)$. Then it is known that

$$
\operatorname{dim} \mathrm{Gr}_{\mathcal{E}}^{-k} \mathcal{R}_{F}=\operatorname{dim} R_{F}^{k}= \begin{cases}1 & (k=0,2) \\ l(\Delta)-3 & (k=1) \\ 0 & (k \geq 3)\end{cases}
$$


See Theorem 4.8 in [5]. The $\mathcal{I}$ - and the $\mathcal{E}$-filtrations on the vector space $\mathcal{R}_{F}$ are described as follows. Let $A^{\prime}(\Delta)=A(\Delta) \backslash\left\{0, m^{(1)}, m^{(2)}, m^{(3)}\right\}$ where $m^{(1)}, m^{(2)}, m^{(3)}$ are any three vertices of $\Delta$. Then we have

$$
\begin{aligned}
\mathcal{R}_{F}=\mathcal{I}_{4} & =\mathcal{E}^{-2} \cong \mathbb{C} 1 \oplus \bigoplus_{m \in A^{\prime}(\Delta)} \mathbb{C} t_{0} t^{m} \oplus \mathbb{C} t_{0} \oplus \mathbb{C} t_{0}^{2}, \\
\mathcal{I}_{1} & \cong \mathbb{C} t_{0} \oplus \mathbb{C} t_{0}^{2}, \quad \mathcal{I}_{3} \cong \mathcal{I}_{1} \oplus \bigoplus_{m \in A^{\prime}(\Delta)} \mathbb{C} t_{0} t^{m}, \\
\mathcal{E}^{0} & =\mathbb{C} 1, \quad \mathcal{E}^{-1} \cong \mathcal{E}^{0} \oplus \bigoplus_{m \in A^{\prime}(\Delta)} \mathbb{C} t_{0} t^{m} \oplus \mathbb{C} t_{0} .
\end{aligned}
$$

As to $\mathcal{I}_{2}$, it depends on the polyhedron $\Delta$. For example, $\mathcal{I}_{2}=\mathcal{I}_{1}$ for the polyhedra $\# 2, \# 3$ in figure 1 , while $\mathcal{I}_{2}=\mathcal{I}_{3}$ for the polyhedra \#4. See Section 8.

For a two-dimensional reflexive polyhedron $\Delta$, there are $l(\Delta)-1$ independent solutions to the $A$-hypergeometric system associated to $\Delta$. Explicit expressions for them can be found in [11, equation (6.22)].

\section{Mixed Hodge structures on $H^{n}\left(\mathbb{T}^{n}, V_{a}^{\circ}\right)$}

Let $\Delta \subset \mathbb{R}^{n}$ be an $n$-dimensional convex integral polyhedron and $F_{a}=\sum a_{m}$ $t^{m} \in \mathbb{L}_{\mathrm{reg}}(\Delta)$. We denote by $V_{a}^{\circ}$ the smooth affine hypersurface in $\mathbb{T}^{n}$ defined by $F_{a}$. We state the result on the (V)MHS on $H^{n}\left(\mathbb{T}^{n}, V_{a}^{\circ}\right)$ due to Batyrev [5] and Stienstra [33]. We remark that $H^{k}\left(\mathbb{T}^{n}, V_{a}^{\circ}\right)=0$ if $k \neq n$ (cf. [5, Theorem 3.4]).

The cokernel of the pull-back $H^{n-1}\left(\mathbb{T}^{n}\right) \rightarrow H^{n-1}\left(V_{a}^{\circ}\right)$ is called the primitive part of the cohomology of $V_{a}^{\circ}$ and denoted by $\mathrm{PH}^{n-1}\left(V_{a}^{\circ}\right)$. From the long exact sequence (2.1), we obtain the following short exact sequence of MHS's:

$$
0 \longrightarrow P H^{n-1}\left(V_{a}^{\circ}\right) \longrightarrow H^{n}\left(\mathbb{T}^{n}, V_{a}^{\circ}\right) \longrightarrow H^{n}\left(\mathbb{T}^{n}\right) \longrightarrow 0
$$

Recall that the MHS on $H^{n}\left(\mathbb{T}^{n}\right)$ is the Tate structure $T(-n)$ (cf. Example 2.1). Therefore, $H^{n}\left(\mathbb{T}^{n}, V_{a}^{\circ}\right)$ is an extension of $T(-n)$ by $P H^{n-1}\left(V_{a}^{\circ}\right)$. 


\subsection{MHS on the primitive part $P H^{n-1}\left(V_{a}^{\circ}\right)$}

Let $R^{+}: S_{\Delta}^{+} \rightarrow \Gamma \Omega_{V_{a}^{o}}^{n-1}$ be the linear map given by

$$
R^{+}\left(t_{0}^{k} t^{m}\right)=\operatorname{Res}_{V_{a}^{\circ}}\left(\frac{(-1)^{k}(k-1) ! \cdot t^{m}}{F^{k}} \omega_{0}\right), \quad \omega_{0}:=\frac{d t_{1}}{t_{1}} \wedge \cdots \wedge \frac{d t_{n}}{t_{n}} .
$$

Theorem 4.1 (Batyrev). (i) $R^{+}$induces an isomorphism

$$
\rho^{+}: \mathcal{R}_{F_{a}}^{+} \stackrel{\cong}{\longrightarrow} P H^{n-1}\left(V_{a}^{\circ}\right)
$$

(ii) Let $\mathcal{W}_{\bullet}^{+}$be the weight filtration on $P H^{n-1}\left(V_{a}^{\circ}\right)$. Then, for $0<i \leq n-1$, we have

$$
\rho^{+}\left(\mathcal{I}_{i}\right)=\mathcal{W}_{n-2+i}^{+}
$$

(iii) Let $\mathcal{F}_{+}^{\bullet}$ be the Hodge filtration on $P H^{n-1}\left(V_{a}^{\circ}\right)$. Then, for $0 \leq i \leq n-1$, we have

$$
\rho^{+}\left(\mathcal{E}_{+}^{i-n}\right)=\mathcal{F}_{+}^{i}
$$

\subsection{MHS on the middle relative cohomology $H^{n}\left(\mathbb{T}^{n}, V_{a}^{\circ}\right)$}

Let $R^{0}: S_{\Delta}^{0} \rightarrow \Gamma \Omega_{\mathbb{T}^{n}}^{n}$ be the linear map given by $R^{0}(1)=\omega_{0}$. Consider the map $R:=R^{+} \oplus R^{0}: \mathbf{S}_{\Delta} \rightarrow \Gamma \Omega_{\mathbb{T}^{n}}^{n} \oplus \Gamma \Omega_{V_{a}^{\circ}}^{n-1}$. Then the following theorem follows from Theorem 4.1 (cf. [33, Theorem 7]).

Theorem 4.2 (Batyrev, Stienstra). (i) $R$ induces an isomorphism

$$
\rho: \mathcal{R}_{F_{a}} \stackrel{\cong}{\longrightarrow} H^{n}\left(\mathbb{T}^{n}, V_{a}^{\circ}\right) .
$$

(ii) Let $\mathcal{W}_{\bullet}$ be the weight filtration on $H^{n}\left(\mathbb{T}^{n}, V_{a}^{\circ}\right)$. Then we have

$$
\begin{aligned}
& \rho\left(\mathcal{I}_{i}\right)=\mathcal{W}_{n-2+i} \quad(0<i \leq n-1), \quad \rho\left(\mathcal{I}_{n+1}\right)=\mathcal{W}_{2 n-2}=\mathcal{W}_{2 n-1}, \\
& H^{n}\left(\mathbb{T}^{n}, V_{a}^{\circ}\right)=\mathcal{W}_{2 n}
\end{aligned}
$$

(iii) Let $\mathcal{F}^{\bullet}$ be the Hodge filtration on $H^{n}\left(\mathbb{T}^{n}, V_{a}^{\circ}\right)$. Then, for $0 \leq i \leq n$, we have

$$
\rho\left(\mathcal{E}^{i-n}\right)=\mathcal{F}^{i}
$$




\subsection{Gauss-Manin connection on $H^{n}\left(\mathbb{T}^{n}, V_{a}^{\circ}\right)$}

Consider the variation of MHS on $H^{n}\left(\mathbb{T}^{n}, V_{a}^{\circ}\right)$ over $\mathbb{L}_{\text {reg }}(\Delta)$. It was studied by Stienstra [33, Section 6].

Lemma 4.1 (Stienstra). The Gauss-Manin connection $\nabla_{\frac{\partial}{\partial a_{i}}}$ on $H^{n}\left(\mathbb{T}^{n}, V_{a}^{\circ}\right)$ corresponds to the operator $\mathcal{D}_{a_{i}}$ on $\mathcal{R}_{F}[\mathbf{a}]$.

Stienstra proved this by considering the de Rham complex $\left(\Omega^{\bullet}\left(\mathbb{T}^{n}\right), d+\right.$ $\left.d F_{a} \wedge\right)$ [33, Section 6]. Here we give a different proof. This is a generalization of Takahashi's argument [34, Lemma 1.8].

Proof. Since $F_{a}$ is $\Delta$-regular, there exists a holomorphic $(n-1)$-form $\psi_{a}$ in an open neighborhood of $V_{a}^{\circ}$ in $\mathbb{T}^{n}$ such that $\omega_{0}=d F_{a} \wedge \psi_{a}$. The restriction of $\psi_{a}$ to $V_{a}^{\circ}$ is equal to $\operatorname{Res}_{V_{a}^{\circ}} \frac{\omega_{0}}{F_{a}}$ and is denoted by $\frac{\omega_{0}}{d F_{a}}$. It is called the Gelfand-Leray form of $\omega_{0}$ (cf. [4, Ch. 10]).

Let $\Gamma_{a} \in H_{n}\left(\mathbb{T}^{n}, V_{a}^{\circ}\right)$. Then one can show that

$$
\frac{\partial}{\partial a_{i}} \int_{\Gamma_{a}} \omega_{0}=-\int_{\partial \Gamma_{a}} \frac{\partial F_{a}}{\partial a_{i}} \frac{\omega_{0}}{d F_{a}} .
$$

Namely, we have $\nabla_{\frac{\partial}{\partial a_{i}}} \rho(1)=\rho\left(\mathcal{D}_{a_{i}} 1\right)$. Since Batyrev [5] have shown that the Gauss-Manin connection $\nabla_{\frac{\partial}{\partial a_{i}}}$ on $P H^{n-1}\left(V_{a}^{\circ}\right)$ corresponds to $\mathcal{D}_{a_{i}}$ under $\rho^{+}$, the lemma follows.

Lemma 4.1 and Proposition 3.1 imply the following

Corollary 4.1 (Stienstra). 1. $H^{n}\left(\mathbb{T}^{n}, V_{a}^{\circ}\right)$ is spanned by $\nabla_{a_{m_{1}}} \cdots \nabla_{a_{m_{k}}} \omega_{0}$ $\left(0 \leq k \leq n, m_{1}, \ldots, m_{k} \in A(\Delta)\right)$.

2. $\omega_{0}$ satisfies the A-hypergeometric system (3.8) with $\partial_{a_{i}}$ replaced by $\nabla_{\partial_{a_{i}}}$.

3. The period integrals $\int_{\Gamma_{a}} \omega_{0}$ of the relative cohomology $H^{n}\left(\mathbb{T}^{n}, V_{a}^{\circ}\right)$ satisfies the $A(\Delta)$-hypergeometric system (3.8). Conversely, a solution of the Ahypergeometric system (3.8) is a period integral.

\section{$5 \quad$ MHS on $H^{3}\left(Z_{a}^{\circ}\right)$}

Throughout the section, $\Delta$ is a two-dimensional reflexive polyhedron and $F_{a} \in \mathbb{L}_{\mathrm{reg}}(\Delta)$ is a $\Delta$-regular Laurent polynomial. 


\subsection{MHS on the cohomology of the threefold}

Consider the affine threefold $Z_{a}^{\circ}$ defined by

$$
Z_{a}^{\circ}=\left\{(t, x, y) \in \mathbb{T}^{2} \times \mathbb{C}^{2} \mid F_{a}(t)+x y=0\right\} .
$$

We compute $H^{3}\left(Z_{a}^{\circ}\right)$ and its (V)MHS following Batyrev [5]. Let us briefly state the results. Details are relegated to Section A.

The Poincaré residue map Res : $H^{4}\left(\mathbb{T}^{2} \times \mathbb{C}^{2} \backslash Z_{a}^{\circ}\right) \cong H^{3}\left(Z_{a}^{\circ}\right)$ is an isomorphism (see equation (A.2)). By Grothendieck [21], $H^{\bullet}\left(\mathbb{T}^{2} \times \mathbb{C}^{2} \backslash Z_{a}^{\circ}\right)$ is isomorphic to the cohomology of the global de Rham complex $\left(\Gamma \Omega_{\mathbb{T}^{2} \times \mathbb{C}^{2}}^{\bullet}\right.$ $\left.\left(* Z_{a}^{\circ}\right), d\right)$ of meromorphic differential forms on $\mathbb{T}^{2} \times \mathbb{C}^{2}$ with poles of arbitrary order on $Z_{a}^{\circ}$. We can show that the homomorphism

$$
R^{\prime}: \mathbf{S}_{\Delta} \rightarrow \Gamma \Omega_{\mathbb{T}^{2} \times \mathbb{C}^{2}}^{4}\left(* Z_{a}^{\circ}\right) ; \quad t_{0}^{k} t^{m} \mapsto \frac{(-1)^{k} k ! t^{m}}{\left(F_{a}+x y\right)^{k+1}} \frac{d t_{1}}{t_{1}} \frac{d t_{2}}{t_{2}} d x d y,
$$

induces an isomorphism $\mathcal{R}_{F_{a}} \cong H^{4}\left(\mathbb{T}^{2} \times \mathbb{C}^{2} \backslash Z_{a}^{\circ}\right)$. Together with the residue map, we obtain an isomorphism $\rho^{\prime}: \mathcal{R}_{F_{a}} \stackrel{\cong}{\rightrightarrows} H^{3}\left(Z_{a}^{\circ}\right)$. The Gauss-Manin connection $\nabla_{\partial_{a_{m}}}$ on $H^{3}\left(Z_{a}^{\circ}\right)$ corresponds to a differentiation by $a_{m}$ on $\Gamma \Omega_{\mathbb{T}^{2} \times \mathbb{C}^{2}}^{4}\left(* Z_{a}^{\circ}\right)$, which in turn corresponds to the derivation $\mathcal{D}_{a_{m}}$ on $\mathcal{R}_{F_{a}}$ (Section A.6).

To compute the weight and the Hodge filtrations, we compactify $Z_{a}^{\circ}$ as a smooth hypersurface in a toric variety (Section A.2). Then we can work out calculation similar to [5, Sections 6 and 8]. (Since our $Z_{a}^{\circ}$ is a hypersurface in $\mathbb{T}^{2} \times \mathbb{C}^{2}$, not in $\mathbb{T}^{4}$, we need some modifications. Especially, we need Mavlyutov's results on Hodge numbers of semiample hypersurfaces in a toric varieties [30].) It turns out that the weight and the Hodge filtrations are given by the $\mathcal{I}$ and the $\mathcal{E}$-filtrations on $\mathcal{R}_{F_{a}}$. The result on MHS of $H^{3}\left(Z_{a}^{\circ}\right)$ is summarized as follows (Theorems A.1, A.2):

$$
\begin{aligned}
& H^{3}\left(Z_{a}^{\circ}\right)=\mathcal{W}_{6}=\mathcal{F}^{0}=\mathcal{F}^{1} \cong \mathcal{R}_{F_{a}} \\
& \mathcal{W}_{3} \cong \mathcal{I}_{1}, \quad \mathcal{W}_{4}=\mathcal{W}_{5} \cong \mathcal{I}_{3} \\
& \mathcal{F}^{2} \cong \mathcal{E}^{-1}, \quad \mathcal{F}^{3} \cong \mathcal{E}^{0}
\end{aligned}
$$

\subsection{Relationship to the relative cohomology}

Let $C_{a}^{\circ}$ be the affine curve in $\mathbb{T}^{2}$ defined by $F_{a}$. Since $\Delta$ is reflexive, it is an affine elliptic curve obtained by deleting $l(\Delta)-1$ points from an elliptic 
curve $C_{a}$. The MHS on the primitive part $P H^{1}\left(C_{a}^{\circ}\right)$ is an extension of $T(-1)^{\oplus(l(\Delta)-4)}$ by $H^{1}\left(C_{a}\right)$ :

$$
0 \rightarrow H^{1}\left(C_{a}\right) \rightarrow P H^{1}\left(C_{a}^{\circ}\right) \rightarrow T(-1)^{\oplus(l(\Delta)-4)} \rightarrow 0 .
$$

This follows from the definition of primitive part and the description of $H^{1}\left(C_{a}^{\circ}\right)$ given in Example 2.2. The MHS on the relative cohomology $H^{2}\left(\mathbb{T}^{2}\right.$, $C_{a}^{\circ}$ ) is an extension (4.1) of $H^{2}\left(\mathbb{T}^{2}\right)=T(-2)$ by $P H^{1}\left(C_{a}\right)$ (cf. Theorem 4.2).

Let $\rho: \mathcal{R}_{F_{a}} \cong H^{2}\left(\mathbb{T}^{2}, C_{a}^{\circ}\right)$ be the isomorphism in Theorem 4.2 and $\omega_{0}=$ $\rho(1) \in H^{2}\left(\mathbb{T}^{2}, C_{a}^{\circ}\right)$. Let

$$
\omega_{a}=\rho^{\prime}(1)=\left[\operatorname{Res} \frac{1}{F_{a}+x y} \frac{d t_{1}}{t_{1}} \frac{d t_{2}}{t_{2}} d x d y\right] \in H^{3}\left(Z_{a}^{\circ}\right) .
$$

Theorem 5.1. The composition of isomorphisms $H^{3}\left(Z_{a}^{\circ} \stackrel{\rho^{\prime-1}}{\rightarrow} \mathcal{R}_{F_{a}} \stackrel{\rho}{\rightarrow} H^{2}\right.$ $\left(\mathbb{T}^{2}, C_{a}^{\circ}\right)$ gives an isomorphism

$$
\rho \circ \rho^{\prime-1}: H^{3}\left(Z_{a}^{\circ} \stackrel{\cong}{\rightrightarrows} H^{2}\left(\mathbb{T}^{2}, C_{a}^{\circ}\right)\right.
$$

of $\mathbb{C}$-vector spaces which sends $\omega_{a}$ to $\omega_{0}$. The filtrations correspond as follows:

$$
\begin{aligned}
& \mathcal{F}^{i+1} H^{3}\left(Z_{a}^{\circ}\right) \stackrel{\cong}{\rightrightarrows} \mathcal{E}^{i-2} \cong \mathcal{F}^{i} H^{2}\left(\mathbb{T}^{2}, C_{a}^{\circ}\right) \quad(i=0,1,2), \\
& \mathcal{W}_{3} H^{3}\left(Z_{a}^{\circ} \stackrel{\cong}{\rightrightarrows} \mathcal{I}_{1} \cong \mathcal{W}_{1} H^{2}\left(\mathbb{T}^{2}, C_{a}^{\circ}\right),\right. \\
& \mathcal{W}_{4} H^{3}\left(Z_{a}^{\circ}\right)=\mathcal{W}_{5} H^{3}\left(Z_{a}^{\circ}\right) \stackrel{\cong}{\rightrightarrows} \mathcal{I}_{3} \cong \mathcal{W}_{2} H^{2}\left(\mathbb{T}^{2}, C_{a}^{\circ}\right)=\mathcal{W}_{3} H^{2}\left(\mathbb{T}^{2}, C_{a}^{\circ}\right) \text {. }
\end{aligned}
$$

Moreover, $\rho \circ \rho^{-1}$ is compatible with the Gauss-Manin connections.

Note that $\mathcal{W}_{1} H^{2}\left(\mathbb{T}^{2}, C_{a}^{\circ}\right)=\mathcal{W}_{1} P H^{1}\left(C_{a}^{\circ}\right) \cong H^{1}\left(C_{a}\right)$. Therefore, it inherits a nondegenerate pairing. The same is true for $\mathcal{W}_{3} H^{3}\left(Z_{a}^{\circ}\right)$, since it is isomorphic to the cohomology $H^{3}\left(Z_{a}\right)$ of a certain smooth compactification $Z_{a}$ of $Z_{a}^{\circ}(\text { cf. Section A.2) })^{8}$.

\section{Analogue of Yukawa coupling}

In this section, $\Delta$ is a 2 -dimensional reflexive polyhedron unless otherwise specified.

\footnotetext{
${ }^{8}$ The divisor $Z_{a} \backslash Z_{a}^{\circ}$ is not smooth but a simple normal crossing divisor. The pull-back $H^{3}\left(Z_{a}\right) \rightarrow \mathcal{W}_{3} H^{3}\left(Z_{a}^{\circ}\right)$, which is always surjective, turns out to be injective. This can be checked by comparing the dimension given in Lemma A.3 and that in Proposition A.5.
} 


\subsection{Definition of Yukawa coupling via affine curves or threefolds}

Let $\Delta$ be a two-dimensional reflexive polyhedron. Let $T^{0} \mathbb{L}_{\text {reg }}(\Delta)$ be the subbundle of the holomorphic tangent bundle $T \mathbb{L}_{\mathrm{reg}}(\Delta)$ of $\mathbb{L}_{\mathrm{reg}}(\Delta)$ generated by $\partial_{a_{0}}$. Consider the family of affine elliptic curves $p: \mathcal{Z} \rightarrow \mathbb{L}_{\mathrm{reg}}(\Delta)$ :

$$
\mathcal{Z}=\left\{(a, t) \in \mathbb{L}_{\mathrm{reg}}(\Delta) \times \mathbb{T}^{2} \mid F_{a}(t)=0\right\}
$$

Let $C_{a}$ be the smooth compactification of the affine curve $C_{a}^{\circ}:=p^{-1}(a)$. Note that we have $\operatorname{Gr}_{\mathcal{F}}^{0} H^{2}\left(\mathbb{T}^{2}, C_{a}^{\circ}\right)=\operatorname{Gr}_{\mathcal{F}}^{0} \mathcal{W}_{1} H^{2}\left(\mathbb{T}^{2}, C_{a}^{\circ}\right)$.

Lemma 6.1. For any $\alpha \in H^{2}\left(\mathbb{T}^{2}, C_{a}^{\circ}\right)$, there exists $\alpha^{\prime} \in \mathcal{W}_{1} H^{2}\left(\mathbb{T}^{2}, C_{a}^{\circ}\right)(\cong$ $\left.H^{1}\left(C_{a}\right)\right)$ such that $[\alpha]=\left[\alpha^{\prime}\right]$ in $\operatorname{Gr}_{\mathcal{F}}^{0} H^{2}\left(\mathbb{T}^{2}, C_{a}^{\circ}\right)=\operatorname{Gr}_{\mathcal{F}}^{0} \mathcal{W}_{1} H^{2}\left(\mathbb{T}^{2}, C_{a}^{\circ}\right)$.

Proof. By (3.11), $\alpha$ is written as

$$
\alpha=\alpha_{2,0} \rho\left(t_{0}^{2}\right)+\sum_{m \in A^{\prime}(\Delta)} \alpha_{1, m} \rho\left(t_{0} t^{m}\right)+\alpha_{1,0} \rho\left(t_{0}\right)+\alpha_{0,0} \omega_{0} .
$$

Take $\alpha^{\prime}=\alpha_{2,0} \rho\left(t_{0}^{2}\right)+c \rho\left(t_{0}\right)$, where $c \in \mathbb{C}$ is arbitrary.

The pairing

$$
H^{2}\left(\mathbb{T}^{2}, C_{a}^{\circ}\right) \times \mathcal{F}^{1} \mathcal{W}_{1} H^{2}\left(\mathbb{T}^{2}, C_{a}^{\circ}\right) \rightarrow \mathbb{C} ; \quad(\alpha, \beta) \mapsto \int_{C_{a}} \alpha^{\prime} \wedge \beta
$$

is independent of the choice of $\alpha^{\prime}$. Recall that $\nabla_{a_{0}} \omega_{0} \in \mathcal{F}^{1} \mathcal{W}_{1} H^{2}\left(\mathbb{T}^{2}, C_{a}^{\circ}\right)$.

Definition 6.1. For $k \geq 1$, we define a map

$$
\operatorname{Yuk}^{(k)}: \underbrace{T \mathbb{L}_{\mathrm{reg}}(\Delta) \times \cdots \times T \mathbb{L}_{\mathrm{reg}}(\Delta)}_{(k-1) \text { times }} \times T^{0} \mathbb{L}_{\mathrm{reg}}(\Delta) \rightarrow \mathcal{O}_{\mathbb{L}_{\mathrm{reg}}(\Delta)}
$$

by

$$
\operatorname{Yuk}^{(k)}\left(A_{1}, \ldots, A_{k-1} ; A_{k}\right)=\int_{C_{a}}\left(\nabla_{A_{1}} \cdots \nabla_{A_{k-1}} \omega_{0}\right)^{\prime} \wedge \nabla_{A_{k}} \omega_{0} .
$$

We call Yuk ${ }^{(3)}$ the Yukawa coupling and denote it by Yuk.

Remark 6.1. Yuk $^{(1)}=\mathrm{Yuk}^{(2)}=0$ by Griffiths' transversality. For $k \geq 4$, $\operatorname{Yuk}^{(k)}\left(A_{1}, \ldots, A_{k-1} ; A_{k}\right)$ is $\mathcal{O}_{\mathbb{L}_{\text {reg }}(\Delta)}$-linear in $A_{1}, A_{k}$ and $\mathbb{C}$-linear in $A_{2}, \ldots$, $A_{k-1}$. For $k=3, \operatorname{Yuk}^{(3)}$ is $\mathcal{O}_{\mathbb{L}_{\mathrm{reg}}(\Delta)}$-multilinear. 
Remark 6.2. Instead of the relative cohomology $H^{2}\left(\mathbb{T}^{2}, C_{a}^{\circ}\right)$, we can use the cohomology $H^{3}\left(Z_{a}^{\circ}\right)$ of the open threefold $Z_{a}^{\circ}$ defined in (5.1), provided that the levels of Hodge and weight filtrations are shifted according to Theorem 5.1 and that the integration on $C_{a}$ is replaced by that on the compact threefold $Z_{a}$ defined in Section A.2.

\subsection{Batyrev's paring}

We would like to give an algebraic description of the Yukawa coupling in terms of the Jacobian ring $R_{F_{a}}$. For that purpose, we recall Batyrev's pairing [5, Section 9]. Let $\Delta$ be an integral convex $n$-dimensional polyhedron and $F_{a} \in \mathbb{L}_{\text {reg }}(\Delta)$ a $\Delta$-regular Laurent polynomial. Denote by $D_{F_{a}}$ the quotient

$$
D_{F_{a}}:=I_{\Delta}^{(1)} /\left(t_{0} F_{a}, t_{0} \theta_{t_{1}} F_{a}, \ldots, t_{0} \theta_{t_{n}} F_{a}\right) \cdot I_{\Delta}^{(1)} .
$$

It is a graded $R_{F_{a}}$-module consisting of the homogeneous pieces $D_{F_{a}}^{i}(1 \leq$ $i \leq n+1)$. We have $D_{F_{a}}^{n+1} \cong \mathbb{C}$. The multiplicative structure of $R_{F_{a}}$-module defines a nondegenerate pairing

$$
\langle,\rangle: R_{F_{a}}^{i} \times D_{F_{a}}^{n+1-i} \rightarrow D_{F_{a}}^{n+1} \cong \mathbb{C} .
$$

Let $H_{F_{a}}$ be the image of the homomorphism $D_{F_{a}} \rightarrow R_{F_{a}}$ induced by the inclusion $I_{\Delta}^{(1)} \hookrightarrow \mathbf{S}_{\Delta}$. Then the above pairing induces a nondegenerate pairing

$$
\{,\}: H_{F_{a}}^{i} \times H_{F_{a}}^{n+1-i} \rightarrow D_{F_{a}}^{n+1} \cong \mathbb{C} ;\{\alpha, \beta\}:=\left\langle\alpha, \beta^{\prime}\right\rangle,
$$

where $\beta^{\prime} \in D_{F_{a}}^{n+1-i}$ is an element such that its image of the homomorphism $D_{F_{a}}^{n+1-i} \rightarrow H_{F_{a}}^{n+1-i}$ is $\beta$.

\subsection{Yukawa coupling in terms of Batyrev's pairing}

Now we come back to the case when $\Delta$ is a two-dimensional reflexive polyhedron. In this case, we have $D_{F_{a}} \cong t_{0} R_{F_{a}}$. We explain that the Yukawa coupling defined in Definition 6.1 is essentially Batyrev's pairing together with a choice (concerning the dependence on the parameter $a$ ) of the isomorphism

$$
\xi_{a}: D_{F_{a}}^{3} \rightarrow \mathbb{C} .
$$

First identify $\mathcal{I}_{1}$ with $H_{F_{a}}$ so that it is compatible with the Hodge decomposition $H^{1}\left(C_{a}\right)=H^{1,0}\left(C_{a}\right) \oplus H^{0,1}\left(C_{a}\right)$ under the isomorphism $\rho: \mathcal{R}_{F_{a}} \rightarrow$ 
$H^{2}\left(\mathbb{T}^{2}, C_{a}^{\circ}\right)$. Then Batyrev's pairing

$$
\{,\}: H_{F_{a}}^{2} \times H_{F_{a}}^{1} \rightarrow D_{F_{a}}^{3} \cong \mathbb{C},
$$

induces an antisymmetric pairing $\langle,\rangle_{\mathcal{I}_{1}}$ on $\mathcal{I}_{1}$. Although we do not have an explicit description of such decomposition $\mathcal{I}_{1}=H_{F_{a}}^{1} \oplus H_{F_{a}}^{2}$, the fact that $H_{F_{a}}^{1}$ and $H_{F_{a}}^{2}$ are one-dimensional makes it possible to find $\langle,\rangle_{\mathcal{I}_{1}}{ }^{9}$. It is given by

$$
\left\langle\alpha_{1,0} t_{0}+\alpha_{2,0} t_{0}^{2}, \beta_{1,0} t_{0}+\beta_{2,0} t_{0}^{2}\right\rangle_{\mathcal{I}_{1}}=\left(-\alpha_{1,0} \beta_{2,0}+\alpha_{2,0} \beta_{1,0}\right) \xi_{a}\left(t_{0}^{3}\right) .
$$

Our choice of $\xi_{a}$ is as follows.

Proposition 6.1. There exists a map $\xi_{a}: D_{F_{a}}^{3} \rightarrow \mathbb{C}$ which is holomorphic in $a \in \mathbb{L}_{\mathrm{reg}}(\Delta)$ and satisfies the following condition:

$$
\left\langle\mathcal{D}_{a_{m}} \alpha, \beta\right\rangle_{\mathcal{I}_{1}}+\left\langle\alpha, \mathcal{D}_{a_{m}} \beta\right\rangle_{\mathcal{I}_{1}}=\partial_{a_{m}}\langle\alpha, \beta\rangle_{\mathcal{I}_{1}}
$$

Proof. Define $\alpha_{m}, \beta_{m} \in \mathbb{C}(a)(m \in A(\Delta))$ and $\gamma, \delta \in \mathbb{C}(a)$ by the following relations in $\mathcal{I}_{1}$ :

$$
t_{0}^{2} t^{m}=\alpha_{m} t_{0}+\beta_{m} t_{0}^{2}, \quad t_{0}^{3}=\gamma t_{0}+\delta t_{0}^{2} .
$$

Then the condition (6.2) is equivalent to

$$
\partial_{a_{m}} \xi_{a}\left(t_{0}^{3}\right)=-\left(2 \alpha_{m}+\delta \beta_{m}+\partial_{a_{0}} \beta_{m}\right) \xi_{a}\left(t_{0}^{3}\right) .
$$

The existence of a solution $\xi_{a}\left(t_{0}^{3}\right)$ to this equation is ensured by the equation

$$
\partial_{a_{n}}\left(2 \alpha_{m}+\delta \beta_{m}+\partial_{a_{0}} \beta_{m}\right)=\partial_{a_{m}}\left(2 \alpha_{n}+\delta \beta_{n}+\partial_{a_{0}} \beta_{n}\right),
$$

which follows from the relations in $\mathcal{I}_{1}$ :

$$
\mathcal{D}_{a_{n}} t_{0}^{2} t^{m}-\mathcal{D}_{a_{m}} t_{0}^{2} t^{n}=0, \quad \mathcal{D}_{a_{n}} t_{0}^{3} t^{m}-\mathcal{D}_{a_{m}} t_{0}^{3} t^{n}=0 .
$$

\footnotetext{
${ }^{9} \mathrm{An}$ isomorphism $\mathcal{I}_{1} \rightarrow H_{F_{a}}^{1} \oplus H_{F_{a}}^{2}$ compatible with the graded quotient is given by $\alpha_{1,0} t_{0}+\alpha_{2,0} t_{0}^{2} \mapsto\left(\alpha_{1,0}-u\right) t_{0} \oplus \alpha_{2,0} t_{0}^{2}$ with some $u$. The induced antisymmetric pairing on $\mathcal{I}_{1}$ turns out to be independent of $u$.
} 
Remark 6.3. The condition (6.2) is equivalent to the following equation for the intersection product on $H^{1}\left(C_{a}\right)$ under the isomorphism $\rho: \mathcal{R}_{F_{a}} \rightarrow$ $H^{2}\left(\mathbb{T}^{2}, C_{a}^{\circ}\right)$ :

$$
\int_{C_{a}} \nabla_{a_{m}} \alpha \wedge \beta+\int_{C_{a}} \alpha \wedge \nabla_{a_{m}} \beta=\partial_{a_{m}} \int_{C_{a}} \alpha \wedge \beta,
$$

which is well known in the context of variations of polarized Hodge structures.

Example 6.1. For the polyhedron \#1 in figure 1, solving (6.3), we obtain

$$
\xi_{a}\left(t_{0}^{3}\right)=\frac{1}{27 a_{1} a_{2} a_{3}+a_{0}^{3}} \times \text { a nonzero constant. }
$$

Batyrev's pairing (6.1) together with the quotient map $\mathcal{R}_{F_{a}} \rightarrow H_{F_{a}}^{2}=$ $R_{F_{a}}^{2}=\mathcal{E}^{-2} / \mathcal{E}^{-1}$ induces a pairing

$$
(,): \mathcal{R}_{F_{a}} \times H_{F_{a}}^{1} \rightarrow D_{F_{a}}^{3} \stackrel{\xi_{a}}{\cong} \mathbb{C} .
$$

Then, by Remark 6.3, we have the equation

$\operatorname{Yuk}^{(k)}\left(A_{1}, \ldots, A_{k-1} ; A_{k}\right)=\left(\mathcal{D}_{A_{1}} \cdots \mathcal{D}_{A_{k-1}} 1, \mathcal{D}_{A_{k}} 1\right) \times$ a nonzero constant.

Here $\mathcal{D}_{A}$ is the shorthand notation for

$$
\mathcal{D}_{A}:=\sum_{m \in A(\Delta)} A_{m} \mathcal{D}_{a_{m}}
$$

where $A=\sum_{m \in A(\Delta)} A_{m} \partial_{a_{m}}$ is a vector field on $\mathbb{L}_{\mathrm{reg}}(\Delta)$.

Example 6.2. Let $\Delta$ be the polyhedron $\# 1$ in figure 1. By (6.4), the Yukawa coupling $\operatorname{Yuk}\left(\partial_{a_{0}}, \partial_{a_{0}} ; \partial_{a_{0}}\right)$ is equal to $\left(\mathcal{D}_{a_{0}}, \mathcal{D}_{a_{0}} 1, \mathcal{D}_{a_{0}} 1\right)=\xi_{a}\left(t_{0}^{3}\right)$ up to nonzero multiplicative constant. Compare with Example 6.4 below.

\subsection{Yukawa coupling and the $A$-hypergeometric system}

Recall the $A$-hypergeometric system introduced in Section 3.4. The following proposition enables us to compute the Yukawa coupling by the $A$-hypergeometric system. (See also Lemma 6.2 in the next subsection.) 
Proposition 6.2. 1. For $k \geq 3$ and $m_{1}, \ldots, m_{k-1} \in A(\Delta)$,

$$
\mathcal{T}_{i} \operatorname{Yuk}^{(k)}\left(\theta_{a_{m_{1}}}, \ldots, \theta_{a_{m_{k-1}}} ; \theta_{a_{0}}\right)=0 \quad(i=0,1,2,3)
$$

2. For a vector $l=\left(l_{m}\right)_{m \in A(\Delta)} \in L(\Delta)$, let $k$ be the order of the differential operator $\square_{l}$. Let us write $\square_{l}$ as

$$
\square_{l}=\partial_{a_{m_{1}}} \cdots \partial_{a_{m_{k}}}-\partial_{a_{n_{1}}} \cdots \partial_{a_{n_{k}}}
$$

Then we have

$$
\operatorname{Yuk}^{(k+1)}\left(\partial_{a_{m_{1}}}, \ldots, \partial_{a_{m_{k}}} ; \partial_{a_{0}}\right)-\operatorname{Yuk}^{(k+1)}\left(\partial_{a_{n_{1}}}, \ldots, \partial_{a_{n_{k}}} ; \partial_{a_{0}}\right)=0 .
$$

Moreover, for $j_{1}, \ldots, j_{h} \in A(\Delta)$, we have

$$
\begin{aligned}
& \operatorname{Yuk}^{(k+h+1)}\left(\partial_{a_{j_{1}}}, \ldots, \partial_{a_{j_{h}}}, \partial_{a_{m_{1}}}, \ldots, \partial_{a_{m_{k}}} ; \partial_{a_{0}}\right) \\
& \quad-\operatorname{Yuk}^{(k+h+1)}\left(\partial_{a_{j_{1}}}, \ldots, \partial_{a_{j_{h}}}, \partial_{a_{n_{1}}}, \ldots, \partial_{a_{n_{k}}} ; \partial_{a_{0}}\right)=0
\end{aligned}
$$

3. For $m, n \in A(\Delta)$,

$$
\begin{aligned}
& \partial_{a_{m}} \operatorname{Yuk}^{(3)}\left(\partial_{a_{0}}, \partial_{a_{n}} ; \partial_{a_{0}}\right)+\partial_{a_{n}} \operatorname{Yuk}^{(3)}\left(\partial_{a_{0}}, \partial_{a_{m}} ; \partial_{a_{0}}\right) \\
& \quad=2 \operatorname{Yuk}^{(4)}\left(\partial_{a_{0}}, \partial_{a_{m}}, \partial_{a_{n}} ; \partial_{a_{0}}\right) .{ }^{10}
\end{aligned}
$$

Proof. Let $\Theta_{a_{m}}:=a_{m} \mathcal{D}_{a_{m}}$.

1. Notice that $\nabla_{\theta_{a_{m_{1}}}} \cdots \nabla_{\theta_{a_{m_{k-1}}}} \omega_{0}=\rho\left(\Theta_{a_{m_{1}}} \cdots \Theta_{a_{m_{k-1}}} 1\right)$ is expressed in the form (cf. (3.11))

$$
\alpha_{2,0} \rho\left(\Theta_{a_{0}}^{2} 1\right)+\sum_{m \in A^{\prime}(\Delta)} \alpha_{1, m} \rho\left(\Theta_{a_{0}} \Theta_{a_{m}} 1\right)+\alpha_{1,0} \rho\left(\Theta_{a_{0}} 1\right)+\alpha_{0,0} \rho(1),
$$

where the coefficients satisfy

$$
\mathcal{T}_{i} \alpha_{2,0}=\mathcal{T}_{i} \alpha_{1,0}=\mathcal{T}_{i} \alpha_{1, m}=\mathcal{T}_{i} \alpha_{0,0}=0, \quad(i=0,1,2) .
$$

By Definition 6.1, we have

$$
\operatorname{Yuk}^{(k)}\left(\theta_{a_{m_{1}}}, \ldots, \theta_{a_{m_{k-1}}} ; \theta_{a_{0}}\right)=\int_{C_{a}} \alpha_{2,0} \rho\left(\Theta_{a_{0}}^{2} 1\right) \wedge \rho\left(\Theta_{a_{0}} 1\right) .
$$

Then the statement follows from Proposition 3.1-2.

The statements 2 and 3 follow from Proposition 3.1-2 and Definition 6.1.

\footnotetext{
${ }^{10}$ This equation is analogous to the case of compact Calabi-Yau threefolds. See [26].
} 


\subsection{Yukawa coupling for quotient family}

Consider the action of $\mathbb{T}^{3}$ on $\mathbb{L}_{\text {reg }}(\Delta)$ :

$$
\mathbb{T}^{3} \times \mathbb{L}_{\mathrm{reg}}(\Delta) \rightarrow \mathbb{L}_{\mathrm{reg}}(\Delta), \quad\left(\lambda_{0}, \lambda_{1}, \lambda_{2}\right) \cdot F_{a}\left(t_{1}, t_{2}\right) \mapsto \lambda_{0} F_{a}\left(\lambda_{1} t_{1}, \lambda_{2} t_{2}\right)
$$

Let $\mathcal{M}(\Delta)$ be the geometric invariant theory quotient of $\mathbb{L}_{\mathrm{reg}}(\Delta)$ by this action $^{11}$. Denote the quotient map by $q: \mathbb{L}_{\mathrm{reg}}(\Delta) \rightarrow \mathcal{M}(\Delta)$.

Since $\mathbb{T}^{3}$ acts as automorphisms on $\mathcal{Z}$, we also have a family of affine curves

$$
\pi: \mathcal{Z} / \mathbb{T}^{3} \rightarrow \mathcal{M}(\Delta)
$$

(Similarly, we can construct the quotient family for the open threefold $Z_{a}^{\circ}$.)

The differential equation (6.5) implies that $\operatorname{Yuk}^{(k)}\left(\theta_{a_{m_{1}}}, \ldots, \theta_{a_{m_{k-1}}} ; \theta_{a_{0}}\right)$ depends on the parameter $a$ only through $\mathbb{T}^{3}$-invariant combinations. Thus we can define the Yukawa coupling for the quotient family as follows. Let $T^{0} \mathcal{M}(\Delta)$ be the subbundle of the holomorphic tangent bundle $T \mathcal{M}(\Delta)$ generated by $q_{*} \theta_{a_{0}}$.

Definition 6.2. We define a map

$$
\operatorname{Yuk}_{\mathcal{M}(\Delta)}^{(k)}: \underbrace{T \mathcal{M}(\Delta) \times \cdots \times T \mathcal{M}(\Delta)}_{(k-1) \text { times }} \times T^{0} \mathcal{M}(\Delta) \rightarrow \mathcal{O}_{\mathcal{M}(\Delta)}
$$

by

$$
\operatorname{Yuk}_{\mathcal{M}(\Delta)}^{(k)}\left(A_{1}, \ldots, A_{k-1} ; A_{k}\right)=\operatorname{Yuk}^{(k)}\left(A_{1}^{\prime}, \ldots, A_{k-1}^{\prime} ; A_{k}^{\prime}\right),
$$

where $A_{i}^{\prime}$ are $\mathbb{T}^{3}$-invariant vector fields on $\mathbb{L}_{\text {reg }}(\Delta)$ such that $q_{*} A_{i}^{\prime}=A_{i}$. The case $k=3$ is called the Yukawa coupling and denoted by $\operatorname{Yuk}_{\mathcal{M}(\Delta)}$. (We may omit the subscript $\mathcal{M}(\Delta)$.)

In the rest of this subsection, we rewrite the differential equations for the Yukawa coupling (Proposition 6.2) obtained in the previous section to the setting of the quotient family. We fix a local coordinates of $\mathcal{M}(\Delta)$ of a particular class: take a basis $\left.l^{(i)}(1 \leq i \leq l(\Delta)-3)\right)$ of the lattice of relations

\footnotetext{
${ }^{11}$ Any $a \in \mathbb{L}_{\mathrm{reg}}(\Delta)$ is stable in the sense of the geometric invariant theory (cf. [5, Definition 10.5]).
} 
$L(\Delta)$. Then

$$
z_{i}=a^{l^{(i)}}, \quad(1 \leq i \leq l(\Delta)-3)
$$

form a local coordinate system on some open subset in $\mathcal{M}(\Delta)$. We use the shorthand notation

$$
\theta_{i}:=\theta_{z_{i}}, \quad \theta_{0}:=q_{*} \theta_{a_{0}}=\sum_{i=1}^{l(\Delta)-3} l_{0}^{(i)} \theta_{i}, \quad \nabla_{i}:=\nabla_{\theta_{z_{i}}}, \quad \nabla_{0}:=\nabla_{\theta_{0}} .
$$

Let $\mathbf{D}$ be the set of differential operators on (some open set of) $\mathcal{M}(\Delta)$, consisting of

$$
\theta_{i_{1}} \cdots \theta_{i_{k}} \mathcal{L}_{l}, \quad\left(k \geq 0,1 \leq i_{1}, \ldots, i_{k} \leq l(\Delta)-3, l \in L(\Delta)\right) .
$$

Here $\mathcal{L}_{l}$ is defined by

$$
\mathcal{L}_{l}=q_{*}\left(\prod_{m ; l_{m}>0} a_{m}^{l_{m}}\right) \square_{l}
$$

Example 6.3. In the case of polyhedron \#1 (see Example 3.3), we have the coordinate $z=a^{(-3,1,1,1)}=\frac{a_{1} a_{2} a_{3}}{a_{0}^{3}}$ and $\theta_{0}:=q_{*} \theta_{a_{0}}=-3 \theta_{z}$. Then

$$
\mathcal{L}_{(-3,1,1,1)}=\theta_{z}^{3}+3 z \theta_{z}\left(3 \theta_{z}+1\right)\left(3 \theta_{z}+2\right),
$$

and $\mathbf{D}$ is generated by $\theta_{z}^{k} \mathcal{L}_{(-3,1,1,1)}(k \geq 0)$.

For $0 \leq i_{1}, \ldots, i_{k} \leq l(\Delta)-3$, we define

$$
Y_{i_{1} \ldots i_{k} ; 0}:=\operatorname{Yuk}^{(k+1)}\left(\theta_{i_{1}}, \ldots, \theta_{i_{k}} ; \theta_{0}\right) .
$$

Proposition 6.2 implies the following:

Lemma 6.2. 1. Let $\mathcal{L} \in \mathbf{D}$ and let $U_{i_{1}, \ldots, i_{k}} \in \mathbb{C}(z)$ be the coefficients of $\theta_{i_{1}} \ldots \theta_{i_{k}}$ in $\mathcal{L}$, i.e.

$$
\mathcal{L}=\sum_{k \geq 1} \sum_{i_{1}, \ldots, i_{k}} U_{i_{1}, \ldots, i_{k}} \theta_{i_{1}} \cdots \theta_{i_{k}} \quad\left(U_{i_{1}, \ldots, i_{k}} \in \mathbb{C}(z)\right) .
$$

Then the Yukawa coupling satisfies

$$
\sum_{k \geq 2} \sum_{i_{1}, \ldots, i_{k}} U_{i_{1} \ldots i_{k}} Y_{i_{1} \ldots i_{k} ; 0}=0
$$

2. For $0 \leq i, j \leq l(\Delta)-3$,

$$
Y_{i j 0 ; 0}=\frac{1}{2}\left(\theta_{i} Y_{j 0 ; 0}+\theta_{j} Y_{i 0 ; 0}\right) .
$$


Example 6.4. Let $\Delta$ be the polyhedron \#1 in figure 1. Applying the above Lemma to the differential operator (6.7), we obtain the equation

$$
(1+27 z) \theta_{z} \operatorname{Yuk}\left(\theta_{z}, \theta_{z} ; \theta_{z}\right)+27 z \operatorname{Yuk}\left(\theta_{z}, \theta_{z} ; \theta_{z}\right)=0,
$$

which implies

$$
\operatorname{Yuk}\left(\theta_{z}, \theta_{z} ; \theta_{z}\right)=-\frac{c}{3(1+27 z)},
$$

where $c$ is some nonzero constant. This result is the same as Example 6.2.

Remark 6.4. Let $t, \partial_{S} F$ be the solutions (3.9) of the $A$-hypergeometric system associated to the polyhedron \#1 in figure 1 . Then we have

$$
\operatorname{Yuk}\left(\partial_{t}, \partial_{t} ; \partial_{t}\right) \propto \partial_{t}^{2} \partial_{S} F
$$

This follows from the multilinearity of Yuk and the fact that

$$
\mathrm{Wr}\left(t, \partial_{S} F\right):=\operatorname{det}\left(\begin{array}{cc}
\theta_{z}^{2} t & \theta_{z} t \\
\theta_{z}^{2} \partial_{S} F & \theta_{z} \partial_{S} F
\end{array}\right)=-\left(\theta_{z} t\right)^{3} \cdot \partial_{t}^{2} \partial_{S} F
$$

is proportional to $\operatorname{Yuk}\left(\theta_{z}, \theta_{z} ; \theta_{z}\right)$ since it satisfies the same differential equation (6.9).

\subsection{Comments on Yukawa coupling in the local A-model and local mirror symmetry}

Let $\Delta$ be a two-dimensional reflexive polyhedron. Consider the twodimensional nonsingular complete smooth fan $\Sigma\left(\Delta^{*}\right)$ whose generators of 1-cones are $A(\Delta) \backslash\{0\}$. Let $\mathbb{P}_{\Sigma\left(\Delta^{*}\right)}$ be the toric surface defined by $\Sigma\left(\Delta^{*}\right)$. For example, $\mathbb{P}_{\Sigma\left(\Delta^{*}\right)}=\mathbb{P}^{2}$ if $\Delta$ is the polyhedron \#1 in figure 1 . Take a basis $C_{i}(1 \leq i \leq l(\Delta)-3)$ of $H_{2}\left(\mathbb{P}_{\Sigma\left(\Delta^{*}\right)}, \mathbb{Z}\right) \cong L(\Delta)$ and let $J_{i}(1 \leq i \leq$ $l(\Delta)-3)$ be the dual basis. Denote by $t_{i}(1 \leq i \leq l(\Delta)-3)$ the coordinates on $H^{2}\left(\mathbb{P}_{\Sigma\left(\Delta^{*}\right)}\right)$ associated to this basis. Let $c_{i}$ be the coefficients of $J_{i}$ in $c_{1}\left(\mathbb{P}_{\Sigma\left(\Delta^{*}\right)}\right)=\sum_{i} c_{i} J_{i}$ and let $J_{i} \cdot J_{j}$ be the intersection numbers.

Let $N_{0, \beta}\left(\mathbb{P}_{\Sigma\left(\Delta^{*}\right)}\right)$ be the genus zero local GW invariant of degree $\beta$, and define $F_{\text {inst }} \mathbb{P}_{\Sigma\left(\Delta^{*}\right)}(t)$ by

$$
F_{\text {inst }}^{\mathbb{P}_{\Sigma\left(\Delta^{*}\right)}}(t)=\sum_{\beta=\sum d_{i} C_{i}} N_{\beta}^{\mathbb{P}_{\Sigma\left(\Delta^{*}\right)}} e^{\sum d_{i} t_{i}} .
$$

Note that $\operatorname{dim} H^{2}\left(\mathbb{P}_{\Sigma\left(\Delta^{*}\right)}\right)=\operatorname{dim} R_{F_{a}}^{1}=l(\Delta)-3$. Let $t_{i}(z)$ be solutions of the $A$-hypergeometric system with a single logarithm, so-called the mirror 
maps, and let $\partial_{S} F$ be a solution with double logarithms. (See [11, equation (6.22)] for definitions of $t_{i}, \partial_{S} F . \Pi_{i}$ there is $t_{i}$ here.) Local mirror symmetry [11] says that, under an appropriate identification between $t_{i}$ 's and $t_{i}(z)$ 's, $\partial_{S} F$ is related to the local GW invariants by

$$
\partial_{S} F=\sum_{i, j=1}^{l(\Delta)-3} \frac{J_{i} \cdot J_{j}}{2} t_{i} t_{j}-\sum_{i=1}^{l(\Delta)-3} c_{i} \partial_{t_{i}} F_{\text {inst }}^{\mathbb{P}_{\Sigma\left(\Delta^{*}\right)}}(t) .
$$

Let $T^{0} H^{2}\left(\mathbb{P}_{\Sigma\left(\Delta^{*}\right)}\right)$ be the one-dimensional subspace of $T H^{2}\left(\mathbb{P}_{\Sigma\left(\Delta^{*}\right)}\right)$ spanned by $\sum_{i} c_{i} \partial_{t_{i}}$. The local A-model Yukawa coupling $\mathrm{Yuk}_{A}$ may be defined as a multilinear map from $T H^{2}\left(\mathbb{P}_{\Sigma\left(\Delta^{*}\right)}\right) \times T H^{2}\left(\mathbb{P}_{\Sigma\left(\Delta^{*}\right)}\right) \times T^{0} H^{2}\left(\mathbb{P}_{\Sigma\left(\Delta^{*}\right)}\right)$ to $\mathcal{O}_{H^{2}\left(\mathbb{P}_{\Sigma\left(\Delta^{*}\right)}\right)}$ given by

$$
\operatorname{Yuk}_{A}\left(\partial_{t_{i}}, \partial_{t_{j}} ; \sum_{l=1}^{l(\Delta)-3} c_{l} \partial_{t_{l}}\right)=\partial_{t_{i}} \partial_{t_{j}} \partial_{S} F .
$$

Example 6.5. Let $\Delta$ be the polyhedron $\# 1$ in figure 1. As in Remark 6.4, the local A-model Yukawa coupling $\mathrm{Yuk}_{A}$ is proportional to the local Bmodel Yukawa coupling Yuk. To get the equality, we set $c=1$ in Example 6.4.

We also see that for the other polyhedra in figure 1, the Yukawa couplings coincide with the local A-model Yukawa couplings $\mathrm{Yuk}_{A}$ under the mirror maps $t_{1}, t_{2}$. See Section 8 .

\section{Holomorphic anomaly equation}

\subsection{Analogue of special Kähler geometry}

We propose an analogue of the special geometry relation for $\mathcal{M}(\Delta)$. Consider the quotient family $\pi: \mathcal{Z} / \mathbb{T}^{3} \rightarrow \mathcal{M}(\Delta)$. We use the same notations $z_{i}, \theta_{0}, \theta_{i}, \nabla_{0}, \nabla_{i}$ as in Section 6.5. Let

$$
\phi:=\nabla_{0} \omega_{0} \in H^{1}\left(C_{z}\right) .
$$

As in (6.8), we set

$$
Y_{i 0 ; 0}=\sqrt{-1} \int_{C_{z}} \nabla_{i} \phi \wedge \phi \quad(0 \leq i \leq l(\Delta)-3) .
$$


We also set

$$
G_{0 \overline{0}}:=-\sqrt{-1} \int_{C_{z}} \phi \wedge \bar{\phi}
$$

This defines a Hermitian metric on $T^{0} \mathcal{M}(\Delta)$ such that the norm of $\theta_{0}$ is $G_{0 \overline{0}}$.

By the definition of $G_{0 \overline{0}}, Y_{00 ; 0}$ and $Y_{i 0 ; 0}$, we have the following

\section{Lemma 7.1.}

$$
\begin{aligned}
& \text { (1) } \nabla_{i} \phi=\frac{\theta_{i} G_{0 \overline{0}}}{G_{0 \overline{0}}} \phi+\frac{Y_{i 0 ; 0}}{G_{0 \overline{0}}} \bar{\phi} \\
& \text { (2) } \bar{\theta}_{j} \frac{\theta_{i} G_{0 \overline{0}}}{G_{0 \overline{0}}}=-\frac{Y_{i 0 ; 0} \bar{Y}_{j 0 ; 0}}{G_{0 \overline{0}}^{2}} .
\end{aligned}
$$

Let

$$
\kappa:=\theta_{0} \frac{\theta_{0} G_{0 \overline{0}}}{G_{0 \overline{0}}}+\left(\frac{\theta_{0} G_{0 \overline{0}}}{G_{0 \overline{0}}}\right)^{2}-\frac{\theta_{0} Y_{00 ; 0}}{Y_{00 ; 0}} \frac{\theta_{0} G_{0 \overline{0}}}{G_{0 \overline{0}}} .
$$

Then

$$
\begin{aligned}
& \text { (3) } \bar{\theta}_{j} \kappa=0 \quad(1 \leq j \leq l(\Delta)-3), \\
& \text { (4) } \nabla_{0}^{2} \phi=\kappa \phi+\frac{\theta_{0} Y_{00 ; 0}}{Y_{00 ; 0}} \nabla_{0} \phi .
\end{aligned}
$$

The second equation is analogous to the special geometry equation [7]. The third equation is an analogue of [40, equation (3.2)].

Example 7.1. Let $\Delta$ be the polyhedron \#1 in figure 1. By comparing the fourth equation of the above lemma and the differential operator (6.7), we have

$$
Y_{00 ; 0}=\frac{9}{1+27 z}, \quad \kappa=-\frac{54 z}{1+27 z}
$$

\subsection{Proposal of local holomorphic anomaly equation}

We propose how to adapt BCOV's holomorphic anomaly equation [7] to the local B-model. Let $\tilde{C}_{n}^{g}(g, n \geq 0)$ be the $n$-point B-model topological string 
amplitude of genus $g$. For $2 g-2+n \leq 0$, we set

$$
\tilde{C}_{0}^{0}=\tilde{C}_{1}^{0}=\tilde{C}_{2}^{0}=0, \quad \tilde{C}_{0}^{1}=0 .
$$

For $2 g-2+n \geq 1$, we put

$$
\tilde{C}_{n+1}^{g}=\left(\theta_{0}-n \frac{\theta_{0} G_{0 \overline{0}}}{G_{0 \overline{0}}}\right) \tilde{C}_{n}^{g} .
$$

For $(g, n)=(0,3)$, let

$$
\tilde{C}_{3}^{0}=Y_{00 ; 0} .
$$

As a holomorphic anomaly equation for $(g, n)=(1,1)$, we propose

$$
\bar{\theta}_{j} \tilde{C}_{1}^{1}=-\frac{1}{2} \bar{\theta}_{j} \frac{\theta_{0} G_{0 \overline{0}}}{G_{0 \overline{0}}}, \quad \text { which implies that } \quad \tilde{C}_{1}^{1}=-\frac{1}{2} \frac{\theta_{0} G_{0 \overline{0}}}{G_{0 \overline{0}}}+f_{1}^{1}(z) .
$$

For $(g, n)=(g, 0)(g \geq 2)$, we propose

$$
\bar{\theta}_{j} \tilde{C}_{0}^{g}=\frac{\bar{Y}_{j 0 ; 0}}{2 G_{0 \overline{0}}^{2}}\left(\tilde{C}_{2}^{g-1}+\sum_{h_{1}+h_{2}=g} \tilde{C}_{1}^{h_{1}} \tilde{C}_{1}^{h_{2}}\right) .
$$

For $g \geq 2, \tilde{C}_{0}^{g}$ can be solved by the Feynman diagram method as in [7] or Yamaguchi-Yau's polynomial method as in [40].

\subsection{Solution by Feynman diagram [7]}

Define the propagator $S^{00}$ by the differential equation $\bar{\theta}_{j} S^{00}=\frac{\bar{Y}_{j 0 ; 0}}{G_{0 \overline{0}}^{2}}$. It is easily solved by Lemma 7.1-(2):

$$
S^{00}=-\frac{1}{Y_{00 ; 0}} \frac{\theta_{0} G_{0 \overline{0}}}{G_{0 \overline{0}}}+f_{s}(z),
$$

where $f_{s}(z)$ is a meromorphic function in $z$. Put $\Delta_{00}:=-1 / S^{00}$. Then assuming (7.3), (7.4) and (7.5), we can show that

$$
\bar{\theta}_{j} \exp \left[-\frac{1}{2 \lambda^{2}} \Delta_{00} x^{2}+\frac{1}{2} \log \frac{\Delta_{00}}{\lambda^{2}}+\sum_{n, g \geq 0} \frac{\lambda^{2 g-2}}{n !} \tilde{C}_{n}^{g} x^{n}\right]=0 .
$$

This implies that $\tilde{C}_{0}^{g}(g \geq 2)$ can be computed as a sum over Feynman diagrams of genus $g$. The difference from the one given in [7] is that there is only one propagator, $S^{00}$. 


\subsection{Solution by Yamaguchi-Yau's method [40]}

Let

$$
A=\frac{\theta_{0} G_{0 \overline{0}}}{G_{0 \overline{0}}} .
$$

From the above Feynmann diagram method and the fact that $\theta_{0} A \in \mathbb{C}(z)[A]$ (see Lemma 7.1-(3)), it follows that $\tilde{C}_{n}^{g}$ is a polynomial of degree $3 g-3+n$ in $\mathbb{C}(z)[A]$. Moreover, it satisfies

$$
\frac{\partial \tilde{C}_{0}^{g}}{\partial A}=-\frac{1}{2 Y_{00 ; 0}}\left(\tilde{C}_{2}^{g-1}+\sum_{h_{1}+h_{2}=g} \tilde{C}_{1}^{h_{1}} \tilde{C}_{1}^{h_{2}}\right) .
$$

Example 7.2. For $(g, n)=(1,1),(1,2)$ and $(2,0)$, we have

$$
\begin{aligned}
\tilde{C}_{1}^{1}= & -\frac{1}{2} A+f_{1}^{1}(z), \quad \tilde{C}_{2}^{1}=A^{2}+A\left(-\frac{\theta_{0} Y_{00 ; 0}}{2 Y_{00 ; 0}}-f_{1}^{1}\right)-\frac{\kappa}{2}+\theta_{0} f_{1}^{1}, \\
\tilde{C}_{0}^{2}= & -\frac{1}{2 Y_{00 ; 0}}\left[\frac{5}{12} A^{3}-\left(\frac{\theta_{0} Y_{00 ; 0}}{4 Y_{00 ; 0}}+f_{1}^{1}\right) A^{2}\right. \\
& \left.+\left(-\frac{\kappa}{2}+\theta_{0} f_{1}^{1}+\left(f_{1}^{1}\right)^{2}\right) A\right]+f_{2}(z) .
\end{aligned}
$$

Example 7.3. Let $\Delta$ be the polyhedron \#1 in figure 1. We checked that $\tilde{C}_{1}^{1}, \tilde{C}_{0}^{2}$ give the correct local $\mathrm{GW}$ invariants of $\mathbb{P}^{2}$ at least in small degrees. The holomorphic ambiguities are

$$
f_{1}^{1}(z)=\frac{1+54 z}{4(1+27 z)}, \quad f_{2}(z)=\frac{(3 / 40) z+(783 / 80) z^{2}+(3645 / 8) z^{3}}{(1+27 z)^{2}} .
$$

The holomorphic limit is

$$
G_{0 \overline{0}} \rightarrow \theta_{z} t
$$

\subsection{Witten's geometric quantization approach}

First recall Witten's geometric quantization and its implication for holomorphic anomaly equation [39]. Let $W=\mathbb{R}^{2 N}$ be a vector space equipped with the standard symplectic form and let $L \rightarrow W$ be a complex line bundle whose connection 1 -form is the canonical 1 -form. Let $\mathcal{M}$ be the space of complex structures on $W$. To each complex structure $J \in \mathcal{M}$, associate the holomorphic polarization $\mathcal{H}_{J}$ which is a subspace of the space of square integrable sections $\Gamma(W, L)$ consisting of "holomorphic" ones. Then an infinite dimensional bundle $\mathcal{H} \rightarrow \mathcal{M}$ is obtained. Witten found a projectively flat 
connection on $\mathcal{H}$. His claim is that if this is applied to the case where $W=H^{3}\left(X^{\vee}, \mathbb{R}\right)$ is the cohomology of a Calabi-Yau threefold $X^{\vee}$, then BCOV's holomorphic anomaly equation appears as the condition for the flatness of a section of $\mathcal{H}$.

We apply Witten's idea to the case when $W=\mathcal{W}_{1} H^{1}\left(C_{z}^{\circ}, \mathbb{R}\right)=H^{1}\left(C_{z}, \mathbb{R}\right)$ and $\mathcal{M}=\mathcal{M}(\Delta)$. (To be precise, $\mathcal{M}(\Delta)$ is not the space of complex structures of $W$ but it is larger in general. However, this point does not matter in the following argument.) Take $\phi, \bar{\phi}$ defined in (7.1) as a basis of $W_{\mathbb{C}}=\mathcal{W}_{1} H^{1}\left(C_{z}^{\circ}\right)=H^{1}\left(C_{z}\right)$ and let $x, \bar{x}$ be the associated complex coordinates. $W$ has a symplectic form $\sqrt{-1} G_{0 \overline{0}} d x \wedge d \bar{x}$ given by the intersection product. Consider the trivial line bundle $L=\mathbb{C} \times W$ with the connection

$$
\delta+\frac{1}{2} G_{0 \overline{0}}(x d \bar{x}-\bar{x} d x) .
$$

Here we use $\delta$ to denote the differential on $W$. Then the holomorphic polarization $\mathcal{H}_{z}(z \in \mathcal{M}(\Delta))$ is as follows:

$$
\begin{aligned}
\mathcal{H}_{z} & =\left\{\Phi \in \Gamma(W, L) \mid\left(\bar{\delta}_{\bar{x}}+\frac{G_{0 \overline{0}}}{2} x\right) \Phi=0\right\} \\
& =\left\{\Phi \in \Gamma(W, L) \mid \Phi=\varphi(x) e^{-\left(G_{0 \overline{0}} / 2\right) x \bar{x}}\right\} .
\end{aligned}
$$

Mimicking Witten's result, we can show that

$$
\theta_{j} \mathcal{H} \subset \mathcal{H}, \quad\left(\bar{\theta}_{j}-\frac{\bar{Y}_{j 0 ; 0}}{2 G_{0 \overline{0}}^{2}}\left(\delta_{x}-\frac{G_{0 \overline{0}}}{2} \bar{x}\right)^{2}\right) \mathcal{H} \subset \mathcal{H} .
$$

Moreover, these make a projectively flat connection on $\mathcal{H}$.

If we regard

$$
\exp \left[\sum_{n, g \geq 0} \frac{\lambda^{2 g-2+n}}{n !} \tilde{C}_{n}^{g} x^{n}\right] \times e^{-\left(G_{0 \overline{0}} / 2\right) x \bar{x}}
$$

as a section of $\mathcal{H}$, then the condition that it is a flat section results in the following equation:

$$
\bar{\theta}_{j} \tilde{C}_{n}^{g}=\frac{\bar{Y}_{j 0 ; 0}}{2 G_{0 \overline{0}}^{2}}\left(\tilde{C}_{n+2}^{g-1}+\sum_{\substack{h_{1}+h_{2}=g, 0 \leq m \leq n}}\left(\begin{array}{c}
n \\
m
\end{array}\right) \tilde{C}_{m+1}^{h_{1}} \tilde{C}_{n-m+1}^{h_{2}}\right) .
$$




\section{Examples}

In this section, we consider the polyhedra \#2, 3,4 in figure 1 .

\section{$8.1 \quad \mathbb{F}_{0}$ case}

Let $\Delta$ be the polyhedron \#2 in figure 1

$$
\Delta=\text { the convex hull of }\{(1,0),(0,1),(-1,0),(0,-1)\} \text {. }
$$

\section{$\Delta$-regularity condition}

The $\Delta$-regularity condition for $F \in \mathbb{L}(\Delta)$ is as follows:

$$
\begin{aligned}
& F\left(t_{1}, t_{2}\right)=a_{0}+a_{1} t_{1}+a_{2} t_{2}+\frac{a_{3}}{t_{1}}+\frac{a_{4}}{t_{2}}, \\
& a_{1} a_{2} a_{3} a_{4} \neq 0, \quad\left(a_{0}^{2}-4 a_{1} a_{3}-4 a_{2} a_{4}\right)^{2}-64 a_{1} a_{2} a_{3} a_{4} \neq 0 .
\end{aligned}
$$

\section{$\mathcal{R}_{F}$ and filtrations}

We have

$$
\mathcal{R}_{F} \cong \mathbb{C} 1 \oplus \mathbb{C} t_{0} \oplus \mathbb{C} t_{0} t_{1} \oplus \mathbb{C} t_{0}^{2} .
$$

The $\mathcal{I}$-filtration and the $\mathcal{E}$-filtration are as follows:

$$
\begin{aligned}
& \mathcal{I}_{1}=\mathcal{I}_{2}=\mathbb{C} t_{0} \oplus \mathbb{C} t_{0}^{2}, \quad \mathcal{I}_{3}=\mathcal{I}_{1} \oplus \mathbb{C} t_{0} t_{1}, \quad \mathcal{I}_{4}=\mathcal{R}_{F} . \\
& \mathcal{E}^{0}=\mathbb{C} 1, \quad \mathcal{E}^{-1}=\mathcal{E}^{0} \oplus \mathbb{C} t_{0} \oplus \mathbb{C} t_{0} t_{1}, \quad \mathcal{E}^{-2}=\mathcal{R}_{F} .
\end{aligned}
$$

\section{MHS}

By Theorem 4.2 and (3.11),

$$
\begin{gathered}
H^{2}\left(\mathbb{T}^{2}, C_{a}^{\circ}\right)=\mathbb{C} \omega_{0} \oplus P H^{1}\left(C_{a}^{\circ}\right), \quad P H^{1}\left(C_{a}^{\circ}\right)=\mathbb{C} \rho\left(t_{0}\right) \oplus \mathbb{C} \rho\left(t_{0} t_{1}\right) \oplus \mathbb{C} \rho\left(t_{0}^{2}\right) . \\
\mathcal{W}_{1}=\mathbb{C} \rho\left(t_{0}\right) \oplus \mathbb{C} \rho\left(t_{0}^{2}\right), \quad \mathcal{W}_{2}=\mathcal{W}_{1} \oplus \mathbb{C} \rho\left(t_{0} t_{1}\right), \quad \mathcal{W}_{4}=H^{2}\left(\mathbb{T}^{2}, \mathbb{C}\right) . \\
\mathcal{E}^{0}=\mathbb{C} \omega_{0}, \quad \mathcal{E}^{-1}=\mathcal{E}^{0} \oplus \mathbb{C} \rho\left(t_{0}\right) \oplus \mathbb{C} \rho\left(t_{0} t_{1}\right), \quad \mathcal{E}^{-2}=H^{2}\left(\mathbb{T}^{2}, \mathbb{C}\right)
\end{gathered}
$$




\section{$A$-hypergeometric system}

The lattice of relations $L(\Delta)$ (defined in (3.7)) is generated by two vectors

$$
l^{(1)}=(-2,1,0,1,0), \quad l^{(2)}=(-2,0,1,0,1) .
$$

The $A$-hypergeometric system is generated by the following differential operators:

$$
\begin{aligned}
& \theta_{a_{1}}-\theta_{a_{3}}, \quad \theta_{a_{2}}-\theta_{a_{4}}, \quad \theta_{a_{1}}+\theta_{a_{2}}+\theta_{a_{3}}+\theta_{a_{4}}+\theta_{a_{0}}, \\
& \partial_{a_{1}} \partial_{a_{3}}-\partial_{a_{0}}^{2}, \quad \partial_{a_{2}} \partial_{a_{4}}-\partial_{a_{0}}^{2} .
\end{aligned}
$$

Take

$$
z_{1}=a^{l^{(1)}}=\frac{a_{1} a_{3}}{a_{0}^{2}}, \quad z_{2}=a^{l^{(2)}}=\frac{a_{2} a_{4}}{a_{0}^{2}} .
$$

These are coordinates of an open subset of $\mathcal{M}(\Delta)$. We have $\theta_{0}:=q_{*} \theta_{a_{0}}=$ $-2 \theta_{z_{1}}-2 \theta_{z_{2}}$. With these coordinates, the above $A$-hypergeometric system reduces to the following two differential operators of order 2 :

$$
\begin{aligned}
& \mathcal{L}_{1}=\theta_{1}^{2}-z_{1}\left(-2 \theta_{1}-2 \theta_{2}\right)\left(-2 \theta_{1}-2 \theta_{2}-1\right), \\
& \mathcal{L}_{2}=\theta_{2}^{2}-z_{2}\left(-2 \theta_{1}-2 \theta_{2}\right)\left(-2 \theta_{1}-2 \theta_{2}-1\right)
\end{aligned}
$$

Solutions about $z_{1}=0, z_{2}=0$ are as follows.

$$
\begin{gathered}
\varpi(z ; 0)=1, \\
t_{1}:=\left.\partial_{\rho_{1}} \varpi(z ; \rho)\right|_{\rho=0}=\log z_{1}+2 H\left(z_{1}, z_{2}\right), \\
t_{2}:=\left.\partial_{\rho_{2}} \varpi(z ; \rho)\right|_{\rho=0}=\log z_{2}+2 H\left(z_{1}, z_{2}\right), \\
\partial_{S} F:=\partial_{\rho_{1}} \partial_{\rho_{2}} \varpi(z ; \rho)=\log z_{1} \log z_{2}+\cdots,
\end{gathered}
$$

where

$$
\begin{aligned}
\varpi(z ; \rho) & =\sum_{n_{1}, n_{2} \geq 0} \frac{\left(2 \rho_{1}+2 \rho_{2}\right) 2 n_{1}+2 n_{2}}{\left(\rho_{1}+1\right)_{n_{1}}^{2}\left(\rho_{2}+1\right)_{n_{2}}^{2}} z_{1}^{n_{1}+\rho_{1}} z_{2}^{n_{2}+\rho_{2}}, \\
H\left(z_{1}, z_{2}\right) & =\sum_{\substack{n_{1}, n_{2} \geq 0 \\
\left(n_{1}, n_{2}\right) \neq(0,0)}} \frac{\left(2 n_{1}+2 n_{2}-1\right) !}{n_{1} !^{2} n_{2} !^{2}} z_{1}^{n_{1}} z_{2}^{n_{2}} .
\end{aligned}
$$

\section{Yukawa coupling}

In this case, $\mathbf{D}$ is generated by $\mathcal{L}_{1}, \mathcal{L}_{2}$. Applying Lemma 6.2 to $\mathcal{L}_{1}, \mathcal{L}_{2}, \theta_{0} \mathcal{L}_{1}$, $\theta_{0} \mathcal{L}_{2}$, we obtain first-order partial differential equations for $Y_{i, j: 0}$. Solving 
these equations, we obtain

$$
\begin{aligned}
& Y_{0,0 ; 0}=\frac{8 c}{d\left(z_{1}, z_{2}\right)}, \\
& Y_{1,1 ; 0}=\frac{8 c z_{1}}{d\left(z_{1}, z_{2}\right)}, \quad Y_{1,2 ; 0}=\frac{c\left(1-4 z_{1}-4 z_{2}\right)}{d\left(z_{1}, z_{2}\right)}, \quad Y_{2,2 ; 0}=\frac{8 c z_{2}}{d\left(z_{1}, z_{2}\right)},
\end{aligned}
$$

where $d\left(z_{1}, z_{2}\right)=\left(1-4 z_{1}-4 z_{2}\right)^{2}-64 z_{1} z_{2}$ and $c \in \mathbb{C}$ is a nonzero constant.

\section{Comparison with the local A-model Yukawa coupling}

We show that the Yukawa coupling and the local A-model Yukawa coupling coincide under the mirror map

$$
\operatorname{Yuk}\left(\partial_{t_{\alpha}}, \partial_{t_{\beta}} ;-2 \partial_{t_{1}}-2 \partial_{t_{2}}\right) \propto \partial_{t_{\alpha}} \partial_{t_{\beta}} \partial_{S} F
$$

For this purpose, let us define the "Wronskian" of $t_{1}, t_{2}, \partial_{S} F$ by

$$
\begin{aligned}
\mathrm{Wr}_{i_{1} \ldots i_{k}}\left(t_{1}, t_{2}, \partial_{S} F\right) & :=\operatorname{det}\left(\begin{array}{ccc}
\theta_{i_{1}} \cdots \theta_{i_{k}} t_{1} & \theta_{1} t_{1} & \theta_{2} t_{1} \\
\theta_{i_{1}} \cdots \theta_{i_{k}} t_{2} & \theta_{1} t_{2} & \theta_{2} t_{2} \\
\theta_{i_{1}} \cdots \theta_{i_{k}} \partial_{S} F & \theta_{1} \partial_{S} F & \theta_{2} \partial_{S} F
\end{array}\right) \\
& =\operatorname{det}\left(\begin{array}{cc}
\theta_{1} t_{1} & \theta_{2} t_{1} \\
\theta_{1} t_{2} & \theta_{2} t_{2}
\end{array}\right) \cdot \sum_{\alpha, \beta=1}^{2} \partial_{1} t_{\alpha} \cdot \partial_{2} t_{\beta} \cdot \partial_{t_{\alpha}} \partial_{t_{\beta}} \partial_{S} F
\end{aligned}
$$

We can show that Lemma 6.2 holds if we replace $\operatorname{Yuk}_{i_{1}, \ldots, i_{k} ; 0}$ with $\mathrm{Wr}_{i_{1} \ldots i_{k}}\left(t_{1}\right.$, $\left.t_{2}, \partial_{S} F\right)^{12}$. Therefore $\mathrm{Wr}_{i j}\left(t_{1}, t_{2}, \partial_{S} F\right)$ must be proportional to $Y_{i j ; 0}$. Then (8.2) follows from the multilinearity of Yuk.

${ }^{12}$ The first statement follows from the cofactor expansion of the determinant and the fact that $t_{1}, t_{2}, \partial_{S} F$ are solutions of $\mathcal{L}=0$ for $\mathcal{L} \in \mathbf{D}$ :

$$
\begin{aligned}
& \sum_{i_{1}, \ldots, i_{k}} U_{i_{1} \ldots i_{k}} \mathrm{Wr}_{i_{1} \ldots i_{k}}\left(t_{1}, t_{2}, \partial_{S} F\right)=\operatorname{det}\left(\begin{array}{cc}
\theta_{1} t_{2} & \theta_{2} t_{2} \\
\theta_{1} \partial_{S} F & \theta_{2} \partial_{S} F
\end{array}\right) \\
& \quad \times \mathcal{L} t_{1}-\operatorname{det}\left(\begin{array}{cc}
\theta_{1} t_{1} & \theta_{2} t_{1} \\
\theta_{1} \partial_{S} F & \theta_{2} \partial_{S} F
\end{array}\right) \mathcal{L} t_{2}+\operatorname{det}\left(\begin{array}{cc}
\theta_{1} t_{1} & \theta_{2} t_{1} \\
\theta_{1} t_{2} & \theta_{2} t_{2}
\end{array}\right) \mathcal{L} \partial_{S} F=0 .
\end{aligned}
$$

To prove the second statement, we first solve $\mathcal{L}_{1} *=\mathcal{L}_{2} *=0$ and express $\theta_{1}^{2} *, \theta_{2}^{2} *$ in terms of $\theta_{1} \theta_{2} *, \theta_{1} *, \theta_{2} *\left(*=t_{1}, t_{2}, \partial_{S} F\right)$. Then if we substitute these into $\theta_{1} \mathrm{Wr}_{11}\left(t_{1}, t_{2}, \partial_{S} F\right)-$ $\mathrm{Wr}_{110}\left(t_{1}, t_{2}, \partial_{S} F\right)$, terms cancel each other and we obtain zero. We can prove the other equations similarly. 


\section{Holomorphic ambiguities}

The multiplication constant of $Y_{00 ; 0}$ is $c=1$. From $\mathcal{L}_{1}, \mathcal{L}_{2}$, we obtain

$$
\kappa=\frac{8\left(z_{1}+z_{2}-6\left(z_{1}^{2}+z_{2}^{2}\right)+12 z_{1} z_{2}\right)}{d\left(z_{1}, z_{2}\right)} .
$$

We checked that $\tilde{C}_{1}^{1}, \tilde{C}_{0}^{2}$ give the correct local GW invariants of $\mathbb{F}_{0}$ for small degrees. The holomorphic ambiguities are

$$
f_{1}^{1}(z)=-\frac{1}{12} \frac{\theta_{0} d\left(z_{1}, z_{2}\right)}{d\left(z_{1}, z_{2}\right)}+\frac{1}{6}, \quad f_{2}(z)=\frac{1}{d\left(z_{1}, z_{2}\right)^{2}}\left(\sum_{i, j=0}^{5} b_{i j} z_{1}^{i} z_{2}^{j}\right) .
$$

(The numerator of $f_{2}(z)$ is omitted because it is long.) As the holomorphic limit, we take

$$
G_{0 \overline{0}} \rightarrow 1-\theta_{0} H\left(z_{1}, z_{2}\right) .
$$

\section{$8.2 \quad \mathbb{F}_{1}$ case}

Let $\Delta$ be the polyhedron $\# 3$ in figure 1 :

$$
\Delta=\text { the convex hull of }\{(1,0),(0,1),(-1,0),(-1,-1)\} .
$$

\section{$\Delta$-regularity}

The $\Delta$-regularity condition for $F \in \mathbb{L}(\Delta)$ is as follows:

$$
\begin{aligned}
& F\left(t_{1}, t_{2}\right)=a_{0}+a_{1} t_{1}+a_{2} t_{2}+\frac{a_{3}}{t_{1}}+\frac{a_{4}}{t_{1} t_{2}}, \\
& a_{1} a_{2} a_{3} a_{4} \neq 0, \quad a_{3}\left(a_{0}^{2}-4 a_{1} a_{3}\right)^{2}-a_{2} a_{4}\left(a_{0}^{3}-36 a_{0} a_{1} a_{3}+27 a_{1} a_{2} a_{4}\right) \neq 0 .
\end{aligned}
$$

\section{$\mathcal{R}_{F}, \mathcal{I}$-filtration, $\mathcal{E}$-filtration and MHS}

These are the same as the $\mathbb{F}_{0}$-case.

\section{$A$-hypergeometric system}

The lattice of relations $L(\Delta)$ is generated by two vectors

$$
l^{(1)}=(-2,1,0,1,0), \quad l^{(2)}=(-1,0,1,-1,1) .
$$


The $A$-hypergeometric system is generated by the following differential operators:

$$
\begin{aligned}
& \theta_{a_{1}}-\theta_{a_{3}}-\theta_{a_{4}}, \quad \theta_{a_{2}}-\theta_{a_{4}}, \quad \theta_{a_{1}}+\theta_{a_{2}}+\theta_{a_{3}}+\theta_{a_{4}}+\theta_{a_{0}}, \\
& \partial_{a_{1}} \partial_{a_{3}}-\partial_{a_{0}}^{2}, \quad \partial_{a_{2}} \partial_{a_{4}}-\partial_{a_{0}} \partial_{a_{3}} .
\end{aligned}
$$

Take

$$
z_{1}=a^{l^{(1)}}=\frac{a_{1} a_{3}}{a_{0}^{2}}, \quad z_{2}=a^{l^{(2)}}=\frac{a_{2} a_{4}}{a_{0} a_{3}} .
$$

These are coordinates of an open subset of $\mathcal{M}(\Delta)$. We have $\theta_{0}:=q_{*} \theta_{a_{0}}=$ $-2 \theta_{z_{1}}-\theta_{z_{2}}$.

With these coordinates, the $A$-hypergeometric system reduces to the following two differential operators of order 2 :

$$
\begin{aligned}
& \mathcal{L}_{1}=\theta_{1}\left(\theta_{1}-\theta_{2}\right)-z_{1}\left(-2 \theta_{1}-\theta_{2}\right)\left(-2 \theta_{1}-\theta_{2}-1\right), \\
& \mathcal{L}_{2}=\theta_{2}^{2}-z_{2}\left(-2 \theta_{1}-\theta_{2}\right)\left(\theta_{1}-\theta_{2}\right) .
\end{aligned}
$$

Solutions about $z_{1}=0, z_{2}=0$ are as follows:

$$
\begin{aligned}
\varpi(z ; 0)=1, \\
t_{1}:=\left.\partial_{\rho_{1}} \varpi(z ; \rho)\right|_{\rho=0}=\log z_{1}+2 H\left(z_{1}, z_{2}\right), \\
t_{2}:=\left.\partial_{\rho_{2}} \varpi(z ; \rho)\right|_{\rho=0}=\log z_{2}+H\left(z_{1}, z_{2}\right), \\
\partial_{S} F:=\left(\frac{1}{2} \partial_{\rho_{1}}^{2}+\partial_{\rho_{1}} \partial_{\rho_{2}}\right) \varpi(z ; \rho),
\end{aligned}
$$

where

$$
\begin{aligned}
\varpi(z ; \rho)= & \sum_{\substack{n_{1}, n_{2} \geq 0\\
}} \frac{\left(2 \rho_{1}+\rho_{2}\right)_{2 n_{1}+n_{2}}}{\left(\rho_{1}+1\right)_{n_{1}}\left(\rho_{2}+1\right)_{n_{2}}^{2}} \frac{\Gamma\left(1+\rho_{1}-\rho_{2}\right)}{\Gamma\left(1+\rho_{1}-\rho_{2}+n_{1}-n_{2}\right)} \\
& \times z_{1}^{n_{1}+\rho_{1}} z_{2}^{n_{2}+\rho_{2}}, \\
H\left(z_{1}, z_{2}\right)= & \sum_{\substack{n_{1}, n_{2} \geq 0 \\
n_{1} \geq n_{2}}} \frac{\left(2 n_{1}+n_{2}-1\right) !}{n_{1} !\left(n_{1}-n_{2}\right) ! n_{2} !^{2}}(-1)^{n_{2}} z_{1}^{n_{1}} z_{2}^{n_{2}} .
\end{aligned}
$$

Here $\Gamma(x)$ denotes the Gamma function. 


\section{Yukawa coupling}

$$
\begin{aligned}
& Y_{0,0 ; 0}=\frac{c\left(8-9 z_{2}\right)}{d\left(z_{1}, z_{2}\right)}, \quad Y_{1,1 ; 0}=\frac{c\left(1+4 z_{1}-z_{2}-3 z_{1} z_{2}\right)}{d\left(z_{1}, z_{2}\right)}, \\
& Y_{1,2 ; 0}=\frac{c\left(1-4 z_{1}-z_{2}+6 z_{1} z_{2}\right)}{d\left(z_{1}, z_{2}\right)}, \quad Y_{2,2 ; 0}=-\frac{c\left(z_{2}\left(1+12 z_{1}\right)\right)}{d\left(z_{1}, z_{2}\right)},
\end{aligned}
$$

where $d\left(z_{1}, z_{2}\right)=\left(1-4 z_{1}\right)^{2}-z_{2}\left(1-36 z_{1}+27 z_{1} z_{2}\right)$ and $c \in \mathbb{C}$ is a nonzero constant.

\section{Comparison with local A-model Yukawa coupling}

As in the $\mathbb{F}_{0}$-case, we can show that

$$
\operatorname{Yuk}\left(\partial_{t_{\alpha}}, \partial_{t_{\beta}} ;-2 \partial_{t_{1}}-\partial_{t_{2}}\right) \propto \partial_{t_{\alpha}} \partial_{t_{\beta}} \partial_{S} F \quad(1 \leq \alpha, \beta \leq 2) .
$$

\section{Holomorphic ambiguities}

The multiplication constant of $Y_{00 ; 0}$ is $c=1$ and

$$
\kappa=\frac{2 z_{1}\left(-32+192 z_{1}+282 z_{2}-144 z_{1} z_{2}-486 z_{2}^{2}+243 z_{2}^{3}\right)}{d\left(z_{1}, z_{2}\right)} .
$$

We checked that $\tilde{C}_{1}^{1}, \tilde{C}_{0}^{2}$ give the correct local GW invariants of $\mathbb{F}_{1}$ for small degrees. The holomorphic ambiguities are

$$
f_{1}^{1}(z)=-\frac{1}{12} \frac{\theta_{0} d\left(z_{1}, z_{2}\right)}{d\left(z_{1}, z_{2}\right)}+\frac{1}{6}, \quad f_{2}(z)=\frac{1}{d\left(z_{1}, z_{2}\right)^{2}}\left(\sum_{i, j=0}^{7} b_{i j} z_{1}^{i} z_{2}^{j}\right) .
$$

(The numerator of $f_{2}(z)$ is omitted.) As the holomorphic limit, we take

$$
G_{0 \overline{0}} \rightarrow 1-\theta_{0} H\left(z_{1}, z_{2}\right) .
$$

\section{3 $\mathbb{F}_{2}$-case}

Let $\Delta$ be the polyhedron \#4 in figure 1

$$
\Delta=\text { the convex hull of }\{(1,0),(0,1),(-1,0),(-2,-1)\} .
$$

This $\Delta$ is different from previous examples in that there are one integral point lying on the middle of an edge. This case has several features different from the previous cases. 


\section{$\Delta$-regularity}

The $\Delta$-regularity condition for $F \in \mathbb{L}(\Delta)$ is as follows ${ }^{13}$ :

$$
\begin{aligned}
& F\left(t_{1}, t_{2}\right)=a_{0}+a_{1} t_{1}+a_{2} t_{2}+\frac{a_{3}}{t_{1}}+\frac{a_{4}}{t_{1}^{2} t_{2}}, \\
& a_{1} a_{2} a_{3} a_{4} \neq 0, \quad\left(a_{3}^{2}-4 a_{2} a_{4}\right)\left(\left(a_{0}^{2}-4 a_{1} a_{3}\right)^{2}-64 a_{1}^{2} a_{2} a_{4}\right) \neq 0 .
\end{aligned}
$$

\section{$\mathcal{R}_{F}$ and filtrations}

$$
\mathcal{R}_{F} \cong \mathbb{C} 1 \oplus \mathbb{C} t_{0} \oplus \mathbb{C} \frac{t_{0}}{t_{1}} \oplus \mathbb{C} t_{0}^{2} .
$$

The $\mathcal{I}$-filtration is

$$
\mathcal{I}_{1} \mathbb{C} t_{0} \oplus \mathbb{C} t_{0}^{2}, \quad \mathcal{I}_{2}=\mathcal{I}_{3}=\mathcal{I}_{1} \oplus \mathbb{C} \frac{t_{0}}{t_{1}}, \quad \mathcal{I}_{4}=\mathcal{R}_{F}
$$

The $\mathcal{E}$-filtration is

$$
\mathcal{E}^{0}=\mathbb{C} 1, \quad \mathcal{E}^{-1}=\mathcal{E}^{0} \oplus \mathbb{C} t_{0} \oplus \mathbb{C} \frac{t_{0}}{t_{1}}, \quad \mathcal{E}^{-2}=\mathcal{R}_{F} .
$$

\section{MHS}

$$
\begin{gathered}
H^{2}\left(\mathbb{T}^{2}, C_{a}^{\circ}\right)=\mathbb{C} \omega_{0} \oplus P H^{1}\left(C_{a}^{\circ}\right), \quad P H^{1}\left(C_{a}^{\circ}\right)=\mathbb{C} \rho\left(t_{0}\right) \oplus \mathbb{C} \rho\left(t_{0} / t_{1}\right) \oplus \mathbb{C} \rho\left(t_{0}^{2}\right) . \\
\mathcal{W}_{1}=\mathbb{C} \rho\left(t_{0}\right) \oplus \mathbb{C} \rho\left(t_{0}^{2}\right), \quad \mathcal{W}_{2}=\mathcal{W}_{1} \oplus \mathbb{C} \rho\left(t_{0} / t_{1}\right), \quad \mathcal{W}_{4}=H^{2}\left(\mathbb{T}^{2}, \mathbb{C}\right) . \\
\mathcal{E}^{0}=\mathbb{C} \omega_{0}, \quad \mathcal{E}^{-1}=\mathcal{E}^{0} \oplus \mathbb{C} \rho\left(t_{0}\right) \oplus \mathbb{C} \rho\left(t_{0} / t_{1}\right), \quad \mathcal{E}^{-2}=H^{2}\left(\mathbb{T}^{2}, \mathbb{C}\right) .
\end{gathered}
$$

\section{$A$-hypergeometric system}

The lattice of relations $L(\Delta)$ is generated by two vectors

$$
l^{(1)}=(-2,1,0,1,0), \quad l^{(2)}=(0,0,1,-2,1),
$$

\footnotetext{
${ }^{13}$ In the last equation, the first factor comes from a one-dimensional face and the second factor comes from the two-dimensional face.
} 
and the $A$-hypergeometric system is generated by the following differential operators:

$$
\begin{aligned}
& \theta_{a_{1}}-\theta_{a_{3}}-2 \theta_{a_{4}}, \quad \theta_{a_{2}}-\theta_{a_{4}}, \quad \theta_{a_{1}}+\theta_{a_{2}}+\theta_{a_{3}}+\theta_{a_{4}}+\theta_{a_{0}}, \\
& \partial_{a_{1}} \partial_{a_{3}}-\partial_{a_{0}}^{2}, \quad \partial_{a_{2}} \partial_{a_{4}}-\partial_{a_{3}}^{2} .
\end{aligned}
$$

Take the following local coordinates of $\mathcal{M}(\Delta)$ :

$$
z_{1}=a^{l^{(1)}}=\frac{a_{1} a_{3}}{a_{0}^{2}}, \quad z_{2}=a^{l^{(2)}}=\frac{a_{2} a_{4}}{a_{3}^{2}} .
$$

Then we have $\theta_{0}:=q_{*} \theta_{a_{0}}=-2 \theta_{z_{1}}$.

With these coordinates, the $A$-hypergeometric system reduces to the following two differential operators of order 2 :

$$
\begin{aligned}
& \mathcal{L}_{1}=\theta_{1}\left(\theta_{1}-2 \theta_{2}\right)-z_{1}\left(-2 \theta_{1}\right)\left(-2 \theta_{1}-1\right), \\
& \mathcal{L}_{2}=\theta_{2}^{2}-z_{2}\left(\theta_{1}-2 \theta_{2}\right)\left(\theta_{1}-2 \theta_{2}-1\right)
\end{aligned}
$$

Solutions about $z_{1}=0, z_{2}=0$ are as follows:

$$
\begin{gathered}
\varpi(z ; 0)=1, \\
t_{1}:=\left.\partial_{\rho_{1}} \varpi(z ; \rho)\right|_{\rho=0}=\log z_{1}+H\left(z_{1}, z_{2}\right)-G\left(z_{2}\right), \\
t_{2}:=\left.\partial_{\rho_{2}} \varpi(z ; \rho)\right|_{\rho=0}=\log z_{2}+2 G\left(z_{2}\right), \\
\partial_{S} F:=\left(\partial_{\rho_{1}}^{2}+\partial_{\rho_{1}} \partial_{\rho_{2}}\right) \varpi(z ; \rho),
\end{gathered}
$$

where

$$
\begin{aligned}
\varpi(z ; \rho)= & \sum_{n_{1}, n_{2} \geq 0} \frac{\left(2 \rho_{1}\right)_{2 n_{1}}}{\left(\rho_{1}+1\right)_{n_{1}}\left(\rho_{2}+1\right)_{n_{2}}^{2}} \frac{\Gamma\left(1+\rho_{1}-2 \rho_{2}\right)}{\Gamma\left(1+\rho_{1}-2 \rho_{2}+n_{1}-2 n_{2}\right)} \\
& \times z_{1}^{n_{1}+\rho_{1}} z_{2}^{n_{2}+\rho_{2}}, \\
H\left(z_{1}, z_{2}\right)= & 2 \sum_{\substack{n_{1}, n_{2} \geq 0 \\
n_{1} \geq 2 n_{2}}} \frac{\left(2 n_{1}-1\right) !}{n_{1} !\left(n_{1}-2 n_{2}\right) ! n_{2} !^{!}} z_{1}^{n_{1}} z_{2}^{n_{2}}, \\
G\left(z_{2}\right)= & \sum_{n_{2} \geq 1} \frac{\left(2 n_{2}-1\right) !}{n_{2} !^{2}} z_{2}^{n_{2}} .
\end{aligned}
$$




\section{Yukawa coupling}

$$
Y_{1,1 ; 0}=\frac{2 c}{d\left(z_{1}, z_{2}\right)}, \quad Y_{1,2 ; 0}=\frac{c\left(1-4 z_{1}\right)}{d\left(z_{1}, z_{2}\right)}, \quad Y_{2,2 ; 0}=-\frac{2 c z_{2}\left(1-8 z_{1}\right)}{\left(1-4 z_{2}\right) d\left(z_{1}, z_{2}\right)},
$$

and $Y_{00 ; 0}=4 Y_{11 ; 0}$ where $d\left(z_{1}, z_{2}\right)=\left(1-4 z_{1}\right)^{2}-64 z_{1}^{2} z_{2}$ and $c \in \mathbb{C}$ is a nonzero constant.

\section{Comparison with local A-model Yukawa coupling}

We show that

$$
\operatorname{Yuk}\left(\partial_{t_{\alpha}}, \partial_{t_{\beta}} ;-2 \partial_{t_{1}}\right) \propto \partial_{t_{\alpha}} \partial_{t_{\beta}} \partial_{S} F \quad(1 \leq \alpha, \beta \leq 2)
$$

Note that the Wronskian $\mathrm{Wr}_{i_{1} \ldots i_{k}}\left(t_{1}, t_{2}, \partial_{S} F\right)$ defined as in (8.3) is divisible by $\theta_{2} t_{2}$ due to the fact that $t_{2}$ does not depend on $z_{1}$. We define the modified Wronskian ${ }^{14}$ by

$$
\begin{aligned}
\mathrm{Wr}_{i_{1} \ldots i_{k}}^{\prime}\left(t_{1}, t_{2}, \partial_{S} F\right) & :=\mathrm{Wr}_{i_{1} \ldots i_{k}}\left(t_{1}, t_{2}, \partial_{S} F\right) / \theta_{2} t_{2} \\
& =\theta_{1} t_{1} \cdot \sum_{\alpha, \beta=1}^{2} \partial_{1} t_{\alpha} \cdot \partial_{2} t_{\beta} \cdot \partial_{t_{\alpha}} \partial_{t_{\beta}} \partial_{S} F .
\end{aligned}
$$

As in the $\mathbb{F}_{0}$-case, Lemma 6.2 holds if we replace $Y_{i_{1} \ldots i_{k}: 0}$ by $\mathrm{Wr}_{i_{1} \ldots i_{k}}^{\prime}\left(t_{1}, t_{2}, \partial_{S}\right.$ $F)$. Therefore $\mathrm{Wr}_{i j}\left(t_{1}, t_{2}, \partial_{S} F\right)$ is proportional to the Yukawa coupling $Y_{i j ; 0}$. Then (8.6) follows from the multi-linearity of Yuk.

\section{Holomorphic ambiguities}

The multiplication constant of $Y_{00 ; 0}$ is $c=1$ and

$$
\kappa=\frac{8 z_{1}\left(1-6 z_{1}+24 z_{1} z_{2}\right)}{d\left(z_{1}, z_{2}\right)}
$$

We checked that $\tilde{C}_{1}^{1}, \tilde{C}_{0}^{2}$ give the correct local GW invariants of $\mathbb{F}_{2}$ for small degrees. The holomorphic ambiguities are

$$
f_{1}^{1}(z)=-\frac{1}{12} \frac{\theta_{0} d\left(z_{1}, z_{2}\right)}{d\left(z_{1}, z_{2}\right)}+\frac{1}{6}, \quad f_{2}(z)=\frac{1}{d\left(z_{1}, z_{2}\right)^{2}}\left(\sum_{i, j=0}^{7} b_{i j} z_{1}^{i} z_{2}^{j}\right) .
$$

\footnotetext{
${ }^{14} \mathrm{~A}$ reason to consider the modified Wronskian in the $\mathbb{F}_{2}$-case is that the Wronskians do not satisfy the statement corresponding to the second one in Lemma 6.2.
} 
(The numerator of $f_{2}(z)$ is omitted.) As the holomorphic limit, we take

$$
G_{0 \overline{0}} \rightarrow 1-\theta_{0} H\left(z_{1}, z_{2}\right) .
$$

\section{Appendix A Mixed Hodge structure of an open threefold}

In this section, $\Delta$ is a 2 -dimensional reflexive polyhedron. Let $F_{a} \in \mathbb{L}_{\mathrm{reg}}(\Delta)$ be a $\Delta$-regular Laurent polynomial. Define $P_{a} \in \mathbb{C}\left[t_{1}^{ \pm}, t_{2}^{ \pm}, x, y\right]$ by

$$
P_{a}\left(t_{1}, t_{2}, x, y\right)=F_{a}\left(t_{1}, t_{2}\right)+x y .
$$

Let $Z_{a}^{\circ}$ be the affine hypersurface in $\mathbb{T}^{2} \times \mathbb{C}^{2}$ defined by $P_{a}$ :

$$
Z_{a}^{\circ}:=\left\{\left(t_{1}, t_{2}, x, y\right) \in \mathbb{T}^{2} \times \mathbb{C}^{2} \mid F_{a}\left(t_{1}, t_{2}\right)+x y=0\right\} .
$$

It is easy to see that the $\Delta$-regularity of $F_{a}$ implies the smoothness of $Z_{a}^{\circ}$.

The goal of the appendix is to give an explicit description of the MHS on $H^{3}\left(Z_{a}^{\circ}\right)$. First, we show that $H^{3}\left(Z_{a}^{\circ}\right) \cong \mathcal{R}_{F_{a}}$. Next we compactify $Z_{a}^{\circ}$ as a hypersurface in a smooth toric variety. Then using this compactification, we compute the Hodge and weight filtrations on $H^{3}\left(Z_{a}^{\circ}\right)$. We use Batyrev's method for affine hypersurfaces in algebraic tori [5, Sections $6-8]$ with some modifications.

\section{A.1 Middle cohomology $H^{3}\left(Z_{a}^{\circ}\right)$}

We have a long exact sequence

$$
\begin{aligned}
\cdots & \rightarrow H^{4}\left(\mathbb{T}^{2} \times \mathbb{C}^{2}\right) \rightarrow H^{4}\left(\mathbb{T}^{2} \times \mathbb{C}^{2} \backslash Z_{a}^{\circ}\right) \stackrel{\text { Res }}{\longrightarrow} H^{3}\left(Z_{a}^{\circ}\right) \\
& \rightarrow H^{5}\left(\mathbb{T}^{2} \times \mathbb{C}^{2}\right) \rightarrow \cdots .
\end{aligned}
$$

Since $H^{4}\left(\mathbb{T}^{2} \times \mathbb{C}^{2}\right)=H^{5}\left(\mathbb{T}^{2} \times \mathbb{C}^{2}\right)=0$, the Poincaré residue map Res : $H^{4}$ $\left(\mathbb{T}^{2} \times \mathbb{C}^{2} \backslash Z_{a}^{\circ}\right) \rightarrow H^{3}\left(Z_{a}^{\circ}\right)$ is an isomorphism.

In the rest of this subsection, $t^{m}$ stands for $t_{1}^{m_{1}} t_{2}^{m_{2}}$. By Grothendieck [21], $H^{\bullet}\left(\mathbb{T}^{2} \times \mathbb{C}^{2} \backslash Z_{a}^{\circ}\right)$ is isomorphic to the cohomology of the global de Rham complex $\left(\Gamma \Omega_{\mathbb{T}^{2} \times \mathbb{C}^{2}}^{\circ}\left(* Z_{a}^{\circ}\right), d\right)$ of meromorphic differential forms on $\mathbb{T}^{2} \times \mathbb{C}^{2}$ 
with poles of arbitrary order on $Z_{a}^{\circ}$. Let $R^{\prime}$ be the homomorphism

$$
\left.R^{\prime}: \mathbf{S}_{\Delta} \rightarrow \Gamma \Omega_{\mathbb{T}^{2} \times \mathbb{C}^{2}}^{4} * Z_{a}^{\circ}\right), \quad t_{0}^{k} t^{m} \mapsto \frac{(-1)^{k} k ! t^{m}}{P_{a}^{k+1}} \frac{d t_{1}}{t_{1}} \frac{d t_{2}}{t_{2}} d x d y
$$

Proposition A.1. The map $R^{\prime}$ induces an isomorphism

$$
R^{\prime}: \mathcal{R}_{F_{a}} \cong H^{4}\left(\mathbb{T}^{2} \times \mathbb{C}^{2} \backslash Z_{a}^{\circ}\right) .
$$

Corollary A.1. The map $R^{\prime}$ and the Poincaré residue map give an isomorphism

$$
\rho^{\prime}: \mathcal{R}_{F_{a}} \stackrel{\cong}{\rightrightarrows} H^{3}\left(Z_{a}^{\circ}\right) .
$$

Remark A.1. For $i=0,1,2, \iota^{*}: H^{i}\left(\mathbb{T}^{2} \times \mathbb{C}^{2}\right) \rightarrow H^{i}\left(Z_{a}^{\circ}\right)$ is an isomorphism where $\iota: Z_{a}^{\circ} \rightarrow \mathbb{T}^{2} \times \mathbb{C}^{2}$ is the inclusion. For $i \geq 4, H^{i}\left(Z_{a}^{\circ}\right)=0$ since $Z_{a}^{\circ}$ is affine.

proof. (of Proposition A.1.) We would like to compute

$$
\frac{\Gamma \Omega_{\mathbb{T}^{2} \times \mathbb{C}^{2}}^{4}\left(* Z_{a}^{\circ}\right)}{d \Gamma \Omega_{\mathbb{T}^{2} \times \mathbb{C}^{2}}^{3}\left(* Z_{a}^{\circ}\right)} \cong H^{4}\left(\mathbb{T}^{2} \times \mathbb{C}^{2} \backslash Z_{a}^{\circ}\right) .
$$

Let

$$
\mathbf{M}_{0}=\mathbb{C}\left[t_{0}, t_{1}^{ \pm}, t_{2}^{ \pm}, x, y\right], \quad \mathbf{M}=\mathbf{M}_{0} / \mathcal{D}_{0}^{\prime} \mathbf{M}_{0}, \quad \mathbf{L}=\mathbb{C}\left[t_{1}^{ \pm}, t_{2}^{ \pm}\right],
$$

where $\mathcal{D}_{0}^{\prime}: \mathbf{M}_{0} \rightarrow \mathbf{M}_{0}$ is defined by

$$
\mathcal{D}_{0}^{\prime}\left(t_{0}^{k} t^{m} x^{m_{3}} y^{m_{4}}\right):=\left\{\begin{array}{ll}
\left(k+t_{0} P_{a}\right) t_{0}^{k} t^{m} x^{m_{3}} y^{m_{4}} & (k>0) \\
\left(1+t_{0} P_{a}\right) t_{0}^{k} t^{m} x^{m_{3}} y^{m_{4}} & (k=0)
\end{array} .\right.
$$

We first rewrite the left-hand side of (A.3) using M. Consider the homomorphism $\Psi_{0}: \mathbf{M}_{0} \rightarrow \Omega_{\mathbb{T}^{2} \times \mathbb{C}^{2}}^{0}\left(* Z_{a}^{\circ}\right)$ given by

$$
\Psi_{0}\left(t_{0}^{k} t^{m} x^{m_{3}} y^{m_{4}}\right)=\left\{\begin{array}{ll}
\frac{(-1)^{k-1}(k-1) ! t^{m} x^{m_{3}} y^{m_{4}}}{P_{a}^{k}}, & (k \geq 1) \\
-t^{m} x^{m_{3}} y^{m_{4}}, & (k=0)
\end{array} .\right.
$$

Then the kernel of $\Psi_{0}$ is $\mathcal{D}_{0}^{\prime} \mathbf{M}_{0}$. Therefore, $\Psi_{0}$ induces an isomorphism $\Psi: \mathbf{M} \stackrel{\cong}{\rightrightarrows} \Omega_{\mathbb{T}^{2} \times \mathbb{C}^{2}}^{0}\left(* Z_{a}^{\circ}\right)$. 
Define the operators $\mathcal{D}_{i}^{\prime}(1 \leq i \leq 4)$ acting on $\mathbf{M}$ by

$$
\begin{aligned}
& \mathcal{D}_{i}^{\prime}\left(t_{0}^{k} t^{m} x^{m_{3}} y^{m_{4}}\right):=\left\{\begin{array}{ll}
\left(m_{i}+t_{0} \theta_{t_{i}} P_{a}\right) t_{0}^{k} t^{m} x^{m_{3}} y^{m_{4}}, & (i=1,2, k>0) \\
m_{i} t_{0}^{k} t^{m} x^{m_{3}} y^{m_{4}}, & (i=1,2, k=0)
\end{array},\right. \\
& \mathcal{D}_{3}^{\prime}\left(t_{0}^{k} t^{m} x^{m_{3}} y^{m_{4}}\right):=\left\{\begin{array}{ll}
\left(m_{3}+t_{0} \theta_{x} P_{a}\right) t_{0}^{k} t^{m} x^{m_{3}-1} y^{m_{4}}, & (k>0) \\
m_{3} t_{0}^{k} t^{m} x^{m_{3}-1} y^{m_{4}}, & (k=0)
\end{array},\right. \\
& \mathcal{D}_{4}^{\prime}\left(t_{0}^{k} t^{m} x^{m_{3}} y^{m_{4}}\right):=\left\{\begin{array}{ll}
\left(m_{4}+t_{0} \theta_{y} P_{a}\right) t_{0}^{k} t^{m} x^{m_{3}} y^{m_{4}-1}, & (k>0) \\
m_{4} t_{0}^{k} t^{m} x^{m_{3}} y^{m_{4}-1}, & (k=0)
\end{array} .\right.
\end{aligned}
$$

Let $e_{1}, \ldots, e_{4}$ be the standard basis on $\mathbb{C}^{4}$. For $I=\left\{i_{1}, \ldots, i_{p}\right\} \subset\{1,2,3,4\}$, let $e_{I}:=e_{i_{1}} \wedge \cdots \wedge e_{i_{p}}$. Then we have an isomorphism

$$
\Psi_{p}: \mathbf{M} \otimes \wedge^{p} \mathbb{C}^{4} \stackrel{\sim}{\rightarrow} \Omega_{\mathbb{T}^{2} \times \mathbb{C}^{2}}^{p}\left(* Z_{a}^{\circ}\right) ; \quad \sum_{i=1}^{4} f_{i} \otimes e_{I} \mapsto \sum_{i=1} \Psi\left(f_{i}\right) \gamma\left(e_{I}\right),
$$

where $\gamma$ is defined by

$$
\gamma\left(e_{i}\right)=\left\{\begin{array}{ll}
\frac{d t_{i}}{t_{i}} & (i=1,2) \\
d x & (i=3) \\
d y & (i=4)
\end{array}, \quad \gamma\left(e_{I}\right)=\gamma\left(e_{i_{1}}\right) \wedge \cdots \wedge \gamma\left(e_{i_{p}}\right) .\right.
$$

If we define $\mathcal{D}^{\prime}: \mathbf{M} \otimes \wedge^{3} \mathbb{C}^{4} \rightarrow \mathbf{M} \otimes \wedge^{4} \mathbb{C}^{4}$ by

$$
\mathcal{D}^{\prime}\left(f_{I} \otimes e_{I}\right):=\sum_{i=1}^{4} \mathcal{D}_{i}^{\prime}\left(f_{I}\right) \gamma\left(e_{i} \wedge e_{I}\right)
$$

we have a commutative diagram

$$
\begin{array}{ccc}
\mathbf{M} \otimes \wedge^{3} \mathbb{C}^{4} & \stackrel{\mathcal{D}^{\prime}}{\rightarrow} & \mathbf{M} \otimes \wedge^{4} \mathbb{C}^{4} \\
\Psi_{3} \downarrow & & \downarrow \Psi_{4} \\
\Omega_{\mathbb{T}^{2} \times \mathbb{C}^{2}}^{3}\left(* Z_{a}^{\circ}\right) & \stackrel{d}{\rightarrow} & \Omega_{\mathbb{T}^{2} \times \mathbb{C}^{2}}^{4}\left(* Z_{a}^{\circ}\right)
\end{array} .
$$

Thus, we have

$$
\frac{\Gamma \Omega_{\mathbb{T}^{2} \times \mathbb{C}^{2}}^{4}\left(* Z_{a}^{\circ}\right)}{\left.d \Gamma \Omega_{\mathbb{T}^{2} \times \mathbb{C}^{2}}^{3} * Z_{a}^{\circ}\right)} \cong \frac{\mathbf{M} \otimes \wedge^{4} \mathbb{C}^{4}}{\mathcal{D}^{\prime} \mathbf{M} \otimes \wedge^{3} \mathbb{C}^{4}} .
$$

Then the proposition follows from the next lemma. 
Lemma A.1. 1. The homomorphism $\mathbf{L}\left[t_{0}\right] \rightarrow \mathbf{M} \otimes \wedge^{4} \mathbb{C}^{4}$ given by $t_{0}^{k} t^{m} \mapsto$ $t_{0}^{k+1} t^{m} \otimes e_{1} \wedge e_{2} \wedge e_{3} \wedge e_{4}$ induces an isomorphism

$$
\mathbf{L}\left[t_{0}\right] / \sum_{i=0}^{2} \mathcal{D}_{i} \mathbf{L}\left[t_{0}\right] \stackrel{\sim}{\longrightarrow} \frac{\mathbf{M} \otimes \wedge^{4} \mathbb{C}^{4}}{\mathcal{D}^{\prime}\left(\mathbf{M} \otimes \wedge^{3} \mathbb{C}^{4}\right)} .
$$

Here $\mathcal{D}_{i}$ are the same as those defined in (3.3).

2. The inclusion $\mathbf{S}_{\Delta} \rightarrow \mathbf{L}\left[t_{0}\right]$ induces an isomorphism

$$
\mathbf{S}_{\Delta} / \sum_{i=0}^{2} \mathcal{D}_{i} \mathbf{S}_{\Delta} \stackrel{\sim}{\longrightarrow} \mathbf{L}\left[t_{0}\right] / \sum_{i=0}^{2} \mathcal{D}_{i} \mathbf{L}\left[t_{0}\right]
$$

Proof of the lemma is by brute force calculation.

\section{A.2 Compactification $Z_{a}$ of $Z_{a}^{\circ}$}

In Sections A.2-A.5, we omit the subscript $a$ from $F_{a}, P_{a}, Z_{a}, Z_{a}^{\circ}, C_{a}^{\circ}$ and $C_{a}$ for simplicity. In Sections A.2 and A.3, $t^{m}$ stands for the Laurent monomial $t_{1}^{m_{1}} t_{2}^{m_{2}} t_{3}^{m_{3}} t_{4}^{m_{4}}$.

We construct a compactification of $Z^{\circ}$ as a semiample smooth hypersurface $Z$ in a four-dimensional toric variety $\mathbf{V}$ such that the divisor $D=Z \backslash Z^{\circ}$ is a simple normal crossing divisor:

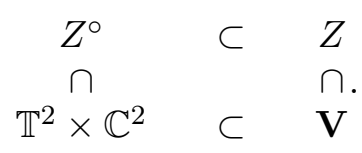

The basic idea is to consider the following slightly modified expression for $P=F+x y$ :

$$
\tilde{P}:=\frac{F\left(t_{1}, t_{2}\right)}{t_{3} t_{4}}+\frac{b_{1}}{t_{4}}+b_{0}, \quad\left(b_{1}, b_{0} \neq 0, F \in \mathbb{L}_{\mathrm{reg}}(\Delta)\right) .
$$

The Newton polyhedron $\tilde{\Delta}$ of $\tilde{P}$ is given by

$$
\begin{aligned}
\tilde{\Delta} & :=\left\{\left(m_{1}, m_{2}, m_{3}, m_{4}\right) \in \mathbb{R}^{4} \mid\right. \\
& \left.m_{3} \leq 0, m_{4} \geq-1, m_{3}-m_{4} \geq 0,\left(m_{1}, m_{2}\right) \in \Delta\left(-m_{3}\right)\right\} .
\end{aligned}
$$


Then by the general theory of the toric variety, we obtain a singular projective toric variety $\mathbf{V}^{\prime}=\operatorname{Proj} \mathbf{S}_{\tilde{\Delta}}$ such that $H^{0}\left(\mathbf{V}^{\prime}, \mathcal{O}(1)\right) \cong \oplus_{m \in A(\tilde{\Delta})} \mathbb{C} t^{m}$. We blow up $\mathbf{V}^{\prime}$ to obtain a smooth toric variety $\mathbf{V}$. A compactification of $Z^{\circ}$ can be obtained as a hypersurface defined by a generic section of the pull-back of $\mathcal{O}(1)$.

Such a $\mathbf{V}$ can be explicitly given as follows. First, let $v_{i} \in \mathbb{Z}^{2}(1 \leq i \leq r$, $r:=l(\Delta)-1)$ be the primitive vectors lying on faces of the dual polyhedron $\Delta^{*}$ of $\Delta$. Let $\Sigma(\Delta)$ be the two-dimensional complete fan spanned by $v_{1}, \ldots, v_{r}$ (see figure 2) and let $\mathbb{P}_{\Sigma(\Delta)}$ be the corresponding smooth toric surface. Then $\mathbb{P}_{\Sigma(\Delta)}$ is a resolution of the singular toric surface Proj $\mathbf{S}_{\Delta}$, and $C^{\circ}$ can be compactified smoothly to $C$ in $\mathbb{P}_{\Sigma(\Delta)}$. Next we set

$$
\begin{aligned}
& \tilde{v}_{i}=\left(\begin{array}{c}
v_{i} \\
-1 \\
0
\end{array}\right) \quad(1 \leq i \leq r), \quad u_{1}=\left(\begin{array}{l}
\overrightarrow{0} \\
1 \\
0
\end{array}\right), \quad u_{2}=\left(\begin{array}{l}
\overrightarrow{0} \\
0 \\
1
\end{array}\right), \quad u_{3}=\left(\begin{array}{c}
\overrightarrow{0} \\
-1 \\
0
\end{array}\right) \\
& u_{4}=\left(\begin{array}{c}
\overrightarrow{0} \\
1 \\
-1
\end{array}\right)
\end{aligned}
$$

Then the 1-cones of the fan $\Sigma_{\mathbf{V}}$ are given by

$$
\nu_{i}=\mathbb{R}_{\geq 0} \tilde{v}_{i}(1 \leq i \leq r), \quad \mu_{i}=\mathbb{R}_{\geq 0} u_{j}(1 \leq j \leq 4)
$$

and the 4-cones of $\Sigma_{\mathbf{V}}$ are given by

$$
\begin{aligned}
{[i, i+1 ; j, j+1]:=} & \mathbb{R}_{\geq 0} \tilde{v}_{i}+\mathbb{R}_{\geq 0} \tilde{v}_{i+1}+\mathbb{R}_{\geq 0} u_{j}+\mathbb{R}_{\geq 0} u_{j+1} \\
& \times(1 \leq i \leq r, 1 \leq j \leq 4) .
\end{aligned}
$$
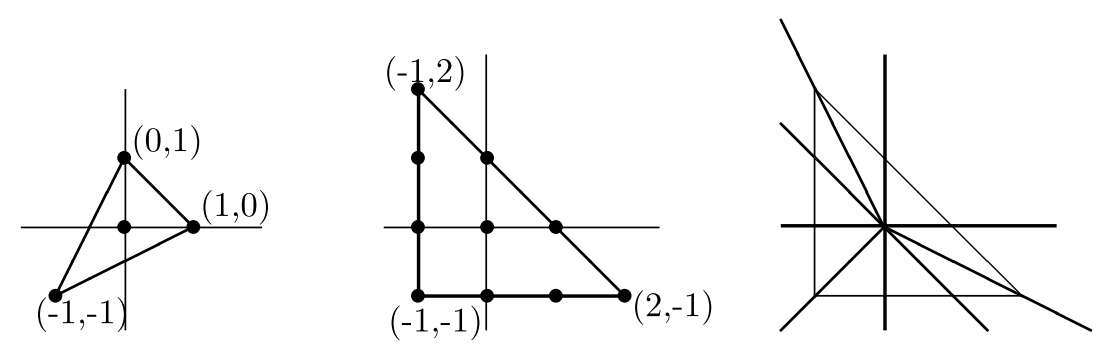

Figure 2: An example of a reflexive polyhedron $\Delta$ (left), its dual polytope $\Delta^{*}$ (middle) and the fan $\Sigma(\Delta)$ (right). 
(For the sake of convenience, we set $\tilde{v}_{r+1}:=\tilde{v}_{1}, u_{5}:=u_{1}$ and $\nu_{r+1}:=\nu_{1}, \mu_{5}$ $:=\mu_{1}$.) The 3 -cones and the 2 -cones are faces of the above 4 -cones. The toric variety $\mathbf{V}$ associated to the fan $\Sigma_{\mathbf{V}}$ is a bundle over the toric surface $\mathbb{P}_{\Sigma(\Delta)}$ whose fiber is the Hirzebruch surface $\mathbb{F}_{1}$.

Let $\mathbb{D}_{i}(1 \leq i \leq r)$ and $\mathbb{E}_{j}(1 \leq j \leq 4)$ be the toric divisors of $\mathbf{V}$ corresponding to the 1 -cones $\nu_{i}$ and $\mu_{j}$, respectively. By a standard computation in the theory of toric varieties (see e.g. [31]), we have

\section{Lemma A.2.}

$$
H^{0}\left(\mathbf{V}, \mathcal{O}\left(\mathbb{E}_{1}+\mathbb{E}_{2}\right)\right)=\bigoplus_{m \in A(\tilde{\Delta})} \mathbb{C} t^{m}
$$

Therefore $\tilde{P}$ in equation (A.4) is a generic section of the line bundle corresponding to the divisor $\mathbb{E}_{1}+\mathbb{E}_{2}$. We define $Z$ to be the hypersurface in $\mathbf{V}$ defined by $\tilde{P}$. We show that if we assume $F$ is $\Delta$-regular and $b_{0} b_{1} \neq 0$, then (1) $Z^{\circ} \subset Z$; (2) $Z$ is smooth; (3) $D=Z / Z^{\circ}$ is a (simple) normal crossing divisor. (1) can be shown as follows. Let $U_{\mu_{1}, \mu_{2}}=\operatorname{Spec}\left[t_{1}^{ \pm 1}, t_{2}^{ \pm 2}, t_{3}, t_{4}\right] \subset \mathbf{V}$ be the open set corresponding to the 2-cone spanned by $\mu_{1}, \mu_{2}$. It is isomorphic to $\mathbb{T}^{2} \times \mathbb{C}^{2}$. The defining equation of $Z$ on $U_{\mu_{1}, \mu_{2}}$ is $F\left(t_{1}, t_{2}\right)+b_{1} t_{3}+$ $b_{0} t_{3} t_{4}$ and this is equal to $P$ if we identify $x=t_{3}, y=b_{1}+b_{0} t_{4}$. We can prove (2) and (3) by looking at the defining equation $P_{\sigma}$ of $Z$ on the open subset $U_{\sigma} \subset \mathbf{V}$ corresponding to each 4-cone $\sigma$.

We end this subsection by listing the Hodge numbers of $Z$.

Lemma A.3. The Hodge numbers $h^{p, q}(Z)=\operatorname{dim} H^{p, q}(Z)$ are

\begin{tabular}{r|rccc} 
& $p=0$ & 1 & 2 & 3 \\
\hline$q=0$ & 1 & 0 & 0 & 0 \\
1 & 0 & $l(\Delta)-1$ & 1 & 0 \\
2 & 0 & 1 & $l(\Delta)-1$ & 0 \\
3 & 0 & 0 & 0 & 1
\end{tabular}.

Proof. By the formula on cohomology of semiample divisors on a toric variety due to Mavlyutov [30, Cor.2.7 ${ }^{15}$, we can explicitly compute the

\footnotetext{
${ }^{15}$ For a semiample toric divisor $X$ in a $d$-dimensional complete simplicial toric variety $\mathbb{P}_{\Sigma}$, Mavlyutov's formula is:

$$
\operatorname{dim} H^{k}\left(\mathbb{P}_{\Sigma}, \Omega_{\mathbb{P}_{\Sigma}}^{l}(X)\right)=\sum_{\delta} l^{*}(\delta)\left(\begin{array}{c}
\operatorname{dim} \delta \\
l-k
\end{array}\right) \cdot \sum_{j=0}^{k}\left(\begin{array}{c}
d-\operatorname{dim} \delta-j \\
k-j
\end{array}\right)(-1)^{k-j} \# \Sigma_{\sigma_{\delta}}(j) .
$$

The sum is over all faces $\delta$ of the polytope $\Delta_{X}$ associated to $X, l^{*}(\delta)$ is the number of interior integral points in the face $\delta, \sigma_{\delta} \in \Sigma_{X}$ is a cone corresponding to the face $\delta$ in the
} 
dimensions of $H^{q}\left(\mathbf{V}, \Omega^{p}\left(\mathbb{E}_{1}+\mathbb{E}_{2}\right)\right)$ and $H^{q}\left(\mathbf{V}, \Omega^{p}\left(2 \mathbb{E}_{1}+2 \mathbb{E}_{2}\right)\right)$. Then we obtain $\operatorname{dim} H^{q}\left(Z, \Omega^{p}\right)$ by exact sequences (as in the proof of the Lefshetz hyperplane theorem $[19, \mathrm{p} .156])$.

\section{A.3 The Hodge filtration}

Let $\mathbb{D}=\sum_{i=1}^{r} \mathbb{D}_{i}+\mathbb{E}_{3}+\mathbb{E}_{4}$. Note that $\mathbb{D}=\mathbf{V} \backslash \mathbb{T}^{2} \times \mathbb{C}^{2}$ and $D=Z / Z^{\circ}=$ $Z \cap \mathbb{D}$.

Proposition A.2. For $p=1,2,3,4$, the residue mapping

$$
H^{4-p}\left(\mathbf{V}, \Omega_{\mathbf{V}}^{p}(\log (Z+\mathbb{D}))\right) \stackrel{\operatorname{Res}_{Z}}{\rightarrow} H^{4-p}\left(Z, \Omega_{Z}^{p-1}(\log D)\right)
$$

is an isomorphism.

Proof. Consider the exact sequence

$$
0 \rightarrow \Omega_{\mathbf{V}}^{p}(\log \mathbb{D}) \rightarrow \Omega_{\mathbf{V}}^{p}(\log (Z+\mathbb{D})) \stackrel{\operatorname{Res}_{Z}}{\rightarrow} \Omega_{Z}^{p-1}(\log D) \rightarrow 0,
$$

and take the cohomology. The vanishings $H^{4-p}\left(\mathbf{V}, \Omega_{\mathbf{V}}^{p}(\log \mathbb{D})\right)=H^{5-p}(\mathbf{V}$, $\left.\Omega_{\mathbf{V}}^{p}(\log \mathbb{D})\right)=0$ imply the proposition.

We use the notation $\Omega_{\mathbf{V}, \mathbb{D}}^{p}(k):=\Omega_{\mathbf{V}}^{p}(\log \mathbb{D}) \otimes \mathcal{O}(k Z)$ for integers $k, p \geq 0$.

\section{Proposition A.3.}

$$
\begin{aligned}
H^{4-p}\left(\mathbf{V}, \Omega^{p}(\log (Z+\mathbb{D}))\right) & \cong \frac{H^{0}\left(\mathbf{V}, \Omega_{\mathbf{V}, \mathbb{D}}^{4}(5-p)\right)}{H^{0}\left(\mathbf{V}, \Omega_{\mathbf{V}, \mathbb{D}}^{4}(4-p)\right)+d H^{0}\left(\mathbf{V}, \Omega_{\mathbf{V}, \mathbb{D}}^{3}(4-p)\right)} \\
H^{0}\left(\mathbf{V}, \Omega^{4}(\log (Z+\mathbb{D}))\right) & \cong H^{0}\left(\mathbf{V}, \Omega_{\mathbf{V}, \mathbb{D}}^{4}(1)\right)
\end{aligned}
$$

fan $\Sigma_{X}$ (which is the fan such that there is a morphism $s: \Sigma \rightarrow \Sigma_{X} ; X$ is a pull-back of an ample divisor by the induced morphism of toric varieties $\left.\mathbb{P}_{\Sigma} \rightarrow \mathbb{P}_{\Sigma_{X}}\right)$, and \# $\Sigma_{\sigma_{\delta}}(j)$ is the number of $j$-cones in $\Sigma_{\sigma_{\delta}}=\left\{s(\tau) \in \Sigma: \tau \in \sigma_{\delta}\right\}$. In the case of the threefold $Z \subset \mathbf{V}$, $\Sigma_{Z}$ is generated by $u_{2}, u_{4}$ and 1-cones $\tilde{v}_{i}$ such that $v_{i}$ are vertices of $\Delta^{*}$. 
Proof. The proposition follows from the exact sequence:

$$
\begin{aligned}
0 & \rightarrow \Omega_{\mathbf{V}}^{p}(\log (Z+\mathbb{D})) \hookrightarrow \Omega_{\mathbf{V}, \mathbb{D}}^{p}(1) \stackrel{d}{\rightarrow} \frac{\Omega_{\mathbf{V}, \mathbb{D}}^{p+1}(2)}{\Omega_{\mathbf{V}, \mathbb{D}}^{p+1}(1)} \stackrel{d}{\rightarrow} \cdots \stackrel{d}{\rightarrow} \frac{\Omega_{\mathbf{V}, \mathbb{D}}^{4}(5-p)}{\Omega_{\mathbf{V}, \mathbb{D}}^{4}(4-p)} \\
& \rightarrow 0
\end{aligned}
$$

and Lemma A.4 below.

Lemma A.4. Let $k$ be a nonnegative integer, $p=1,2,3,4$.

$$
\begin{array}{ll}
\text { 1. } & H^{q}\left(\mathbf{V}, \Omega^{p}\left(\log \left(\mathbb{D}+\mathbb{E}_{1}+\mathbb{E}_{2}\right)\right) \otimes \mathcal{O}(k Z)\right)=0 \quad(q>0), \\
\text { 2. } & H^{q}\left(\mathbf{V}, \Omega^{p}\left(\log \left(\mathbb{D}+\mathbb{E}_{i}\right)\right) \otimes \mathcal{O}(k Z)\right)=0 \quad(q>0, i=1,2), \\
\text { 3. } & H^{q}\left(\mathbf{V}, \Omega_{\mathbf{V}, \mathbb{D}}^{p}(k)\right)=0 \quad(q>0) .
\end{array}
$$

Proof. 1. Let $\mathbb{D}_{\mathbb{T}}:=\mathbb{D}+\mathbb{E}_{1}+\mathbb{E}_{2}$. Note that this is the sum of all toric divisors in $\mathbf{V}$. It is well known that $\Omega_{\mathbf{V}}^{p}\left(\log \mathbb{D}_{\mathbb{T}}\right) \cong \mathcal{O}_{\mathbf{V}} \otimes \wedge^{p} M$ where $M$ is the dual lattice of $N \cong \mathbb{Z}^{4}$ (cf. [31]). On the other hand, since $\mathbb{E}_{1}+\mathbb{E}_{2}$ is semiample, we have $H^{q}\left(\mathbf{V}, \mathcal{O}_{\mathbf{V}}(k Z)\right)=0$ for $q>0$ [30]. Therefore,

$$
H^{q}\left(\mathbf{V}, \Omega_{\mathbf{V}}^{p}\left(\log \mathbb{D}_{\mathbb{T}}\right) \otimes \mathcal{O}_{\mathbf{V}}(k Z)\right) \cong H^{q}\left(\mathbf{V}, \mathcal{O}_{\mathbf{V}}(k Z)\right) \otimes \wedge^{p} M=0 \quad(q>0) .
$$

2. As above, the following vanishing holds:

$$
\begin{aligned}
& H^{q}\left(\mathbb{E}_{2}, \Omega_{\mathbb{E}_{2}}^{p-1}\left(\log \left(\mathbb{D}+\mathbb{E}_{1}\right)\right) \otimes \mathcal{O}_{\mathbb{E}_{2}}(k Z)\right) \\
& \quad \cong H^{q}\left(\mathbb{E}_{2}, \mathcal{O}_{\mathbb{E}_{2}}(k Z)\right) \otimes \wedge^{p-1} \mathbb{Z}^{3}=0 \quad(q>0) .
\end{aligned}
$$

Moreover, the map

$$
H^{0}\left(\mathbf{V}, \Omega_{\mathbf{V}}^{p}\left(\log \mathbb{D}_{\mathbb{T}}\right) \otimes \mathcal{O}_{\mathbf{V}}(k Z)\right) \stackrel{\operatorname{Res}_{\mathbb{E}_{2}}}{\rightarrow} H^{0}\left(\mathbb{E}_{2}, \Omega_{\mathbb{E}_{2}}^{p-1}\left(\log \left(\mathbb{D}+\mathbb{E}_{1}\right)\right) \otimes \mathcal{O}_{\mathbb{E}_{2}}(k Z)\right)
$$

is surjective. Taking the exact sequence of cohomology of the exact sequence

$$
\begin{aligned}
0 \rightarrow \Omega_{\mathbf{V}}^{p}\left(\log \left(\mathbb{D}+\mathbb{E}_{1}\right)\right) & \otimes \mathcal{O}_{\mathbf{V}}(k Z) \rightarrow \Omega_{\mathbf{V}}^{p}\left(\log \mathbb{D}_{\mathbb{T}}\right) \otimes \mathcal{O}_{\mathbf{V}}(k Z) \\
& \stackrel{\operatorname{Res}_{\mathbb{E}_{2}}}{\rightarrow} \Omega_{\mathbb{E}_{2}}^{p-1}\left(\log \left(\mathbb{D}+\mathbb{E}_{1}\right)\right) \otimes \mathcal{O}_{\mathbb{E}_{2}}(k Z) \rightarrow 0,
\end{aligned}
$$

we obtain

$$
H^{q}\left(\mathbf{V}, \Omega^{p}\left(\log \left(\mathbb{D}+\mathbb{E}_{1}\right)\right) \otimes \mathcal{O}(k Z)\right)=0 \quad(q>0) .
$$


The proof for $\mathbb{E}_{2}$ is similar.

3. As above, we can show the vanishing

$$
\begin{aligned}
& H^{q}\left(\mathbb{E}_{1} \cap \mathbb{E}_{2}, \Omega_{\mathbb{E}_{1} \cap \mathbb{E}_{2}}^{p-2}(\log \mathbb{D}) \otimes \mathcal{O}(k Z)\right) \\
& \quad \cong H^{q}\left(\mathbb{E}_{1} \cap \mathbb{E}_{2}, \mathcal{O}_{\mathbb{E}_{1} \cap \mathbb{E}_{2}}(k Z)\right) \otimes \wedge^{p-2} \mathbb{Z}^{2}=0 \quad(q>0),
\end{aligned}
$$

and the surjectivity of the map

$$
\begin{aligned}
& \bigoplus_{i=1,2} H^{0}\left(\mathbf{V}, \Omega_{\mathbf{V}}^{p}\left(\log \left(\mathbb{D}+\mathbb{E}_{i}\right)\right) \otimes \mathcal{O}(k Z)\right) \\
& \stackrel{\operatorname{Res}_{\mathbb{E}_{1}, \mathbb{E}_{2}}}{\rightarrow} H^{0}\left(\mathbb{E}_{1} \cap \mathbb{E}_{2}, \Omega_{\mathbb{E}_{1} \cap \mathbb{E}_{2}}^{p-2}(\log \mathbb{D}) \otimes \mathcal{O}(k Z)\right) .
\end{aligned}
$$

Consider the exact sequence

$$
\begin{aligned}
0 & \rightarrow \Omega_{\mathbf{V}}^{p}(\log \mathbb{D}) \otimes \mathcal{O}(k Z) \rightarrow \bigoplus_{i=1,2} \Omega_{\mathbf{V}}^{p}\left(\log \left(\mathbb{D}+\mathbb{E}_{i}\right)\right) \otimes \mathcal{O}(k Z) \\
& \rightarrow \Omega_{\mathbf{V}}^{p}\left(\log \mathbb{D}_{\mathbb{T}}\right) \otimes \mathcal{O}(k Z) \stackrel{\operatorname{Res}_{\mathbb{E}_{1}} \mathbb{E}_{2}}{\longrightarrow} \Omega_{\mathbb{E}_{1} \cap \mathbb{E}_{2}}^{p-2}(\log \mathbb{D}) \otimes \mathcal{O}(k Z) \rightarrow 0 .
\end{aligned}
$$

Taking the cohomology, we obtain the statement 3 .

Let $\tilde{\Delta}(k)$ be the polyhedron defined by applying (3.1) to $\tilde{\Delta}$ defined in (A.5) and let $\tilde{\Delta}[k]$ be the following four-dimensional polyhedron:

$$
\begin{aligned}
\tilde{\Delta}[k]:=\{ & \left(m_{1}, m_{2}, m_{3}, m_{4}\right) \in \mathbb{R}^{4} \mid \\
& \left(m_{1}, m_{2}\right) \in \Delta\left(-m_{3}-1\right),-k+1 \leq m_{3} \leq 0, m_{4} \geq-k, \\
& \left.m_{3}-m_{4} \geq 0\right\} .
\end{aligned}
$$

Proposition A.4. Let $k$ be a positive integer $k \geq 1$.

$$
\begin{aligned}
& \text { 1. } H^{0}\left(\mathbf{V}, \Omega_{\mathbf{V}, \mathbb{D}}^{4}(k)\right)=\bigoplus_{m \in \tilde{\Delta}(k-1) \cap \mathbb{Z}^{4}} \mathbb{C} \frac{t^{m}}{\tilde{P}^{k}} \frac{d t_{1}}{t_{1}} \frac{d t_{2}}{t_{2}} \frac{d t_{3}}{t_{3}} \frac{d t_{4}}{t_{4}} . \\
& \text { 2. } H^{0}\left(\mathbf{V}, \Omega_{\mathbf{V}, \mathbb{D}}^{3}(k)\right)=\bigoplus_{m \in \tilde{\Delta}[k] \cap \mathbb{Z}^{4}} \mathbb{C} \frac{t^{m}}{\tilde{P}^{k}} \frac{d t_{1}}{t_{1}} \frac{d t_{2}}{t_{2}} \frac{d t_{3}}{t_{3}} \\
& \oplus \bigoplus_{m \in \tilde{\Delta}(k-1) \cap \mathbb{Z}^{4}}\left[\mathbb{C} \frac{t^{m}}{\tilde{P}^{k}} \frac{d t_{1}}{t_{1}} \frac{d t_{2}}{t_{2}} \frac{d t_{4}}{t_{4}} \oplus \mathbb{C} \frac{t^{m}}{\tilde{P}^{k}} \frac{d t_{1}}{t_{1}} \frac{d t_{3}}{t_{3}} \frac{d t_{4}}{t_{4}} \oplus \mathbb{C} \frac{t^{m}}{\tilde{P}^{k}} \frac{d t_{2}}{t_{2}} \frac{d t_{3}}{t_{3}} \frac{d t_{4}}{t_{4}}\right] . \\
& \text { 3. } \frac{H^{0}\left(\mathbf{V}, \Omega_{\mathbf{V}, \mathbb{D}}^{4}(k+1)\right)}{H^{0}\left(\mathbf{V}, \Omega_{\mathbf{V}, \mathbb{D}}^{n}(k)\right)+d H^{0}\left(\mathbf{V}, \Omega_{\mathbf{V}, \mathbb{D}}^{3}(k)\right)} \cong R_{F}^{k},
\end{aligned}
$$


where the isomorphism is induced from the map

$$
\mathbf{S}_{\Delta}^{k} \rightarrow H^{0}\left(\mathbf{V}, \Omega_{\mathbf{V}, \mathbb{D}}^{4}(k+1)\right), \quad t_{0}^{k} t_{1}^{m_{1}} t_{2}^{m_{2}} \mapsto \frac{(-1)^{k} k ! t_{1}^{m_{1}} t_{2}^{m_{2}}}{\tilde{P}^{k+1}\left(t_{3} t_{4}\right)^{k}} \frac{d t_{1}}{t_{1}} \frac{d t_{2}}{t_{2}} \frac{d t_{3}}{t_{3}} \frac{d t_{4}}{t_{4}} .
$$

Proof. The statements 1 and 2 can be shown by calculation of the Cech cohomology associated to the open cover given by the toric fan. The third statement follows from the first and the second.

Theorem A.1. The Hodge filtration on $H^{3}\left(Z^{\circ}\right)$ satisfies

$$
\operatorname{Gr}_{\mathcal{F}}^{p} H^{3}\left(Z^{\circ}\right) \cong R_{F}^{3-p}, \quad(0 \leq p \leq 3) .
$$

Proof. As is well known, there are canonical isomorphisms $\operatorname{Gr}_{\mathcal{F}}^{p} H^{3}\left(Z^{\circ}\right) \cong$ $H^{3-p}\left(Z, \Omega_{Z}^{p}(\log D)\right)$. The theorem follows from Propositions A.2, A.3 and A.4.

\section{A.4 The weight filtrations}

\section{Proposition A.5.}

$$
\begin{aligned}
& \operatorname{dim} \operatorname{Gr}_{6}^{\mathcal{W}}=1, \quad \operatorname{dim} \operatorname{Gr}_{5}^{\mathcal{W}}=0, \\
& \operatorname{dim} \operatorname{Gr}_{4}^{\mathcal{W}}=l(\Delta)-4, \quad \operatorname{dim} \operatorname{Gr}_{3}^{\mathcal{W}}=2 .
\end{aligned}
$$

Proof. The divisor $D=Z \backslash Z^{\circ}$ consists of $r+2$ components. Define $D^{(k)}$ to be the disjoint union of intersections of $k$ components for $k=1,2,3$ and $D^{(0)}:=Z$. Consider the spectral sequence $\mathcal{W} E$ of the hypercohomology $\mathbb{H}^{k}\left(Z, \Omega_{Z}^{\bullet}(\log D)\right)$ associated to the decreasing weight filtration $\mathcal{W}^{-l}:=\mathcal{W}_{l}$. This spectral sequence degenerates at $\mathcal{W} E_{2}$. We have $\mathcal{W} E_{1}^{p, q} \cong H^{2 p+q}\left(D^{(-p)}\right.$, $\mathbb{C})$ and the differential $d_{1}: H^{2 p+q}\left(D^{(-p)}, \mathbb{C}\right) \rightarrow H^{2 p+q+2}\left(D^{(-p-1)}, \mathbb{C}\right)$ is given by the Gysin morphism (see e.g. [37, Corollary 8.33, Proposition 8.34]). Computing the cohomology of $d_{1}$, we obtain the following result:

$$
\begin{array}{r|rccc} 
& p=0 & -1 & -2 & -3 \\
\hline q=0 & 1 & 0 & 0 & 0 \\
1 & 0 & 0 & 0 & 0 \\
2 & 0 & 2 & 0 & 0 \\
3 & 2 & 0 & 0 & 0 \\
4 & 0 & l(\Delta)-4 & 1 & 0 \\
5 & 0 & 0 & 0 & 0 \\
6 & 0 & 0 & 0 & 1
\end{array} .
$$


The dimensions of the graded quotients $\operatorname{Gr}_{-p+3}^{\mathcal{W}} H^{3}\left(Z^{\circ}\right) \cong{ }_{\mathcal{W}} E_{2}^{p, 3-p}$ can be read from this table.

Proposition A.6. The weight filtration on $H^{4}\left(\mathbb{T}^{2} \times \mathbb{C}^{2} \backslash Z^{\circ}\right)$ is

$$
\begin{aligned}
& \mathcal{W}_{8} H^{4}\left(\mathbb{T}^{2} \times \mathbb{C}^{2} \backslash Z^{\circ}\right)=\mathcal{I}_{4}=\mathcal{R}_{F}, \\
& \mathcal{W}_{7} H^{4}\left(\mathbb{T}^{2} \times \mathbb{C}^{2} \backslash Z^{\circ}\right)=\mathcal{W}_{6} H^{4}\left(\mathbb{T}^{2} \times \mathbb{C}^{2} \backslash Z^{\circ}\right)=\mathcal{I}_{3}, \\
& \mathcal{W}_{5} H^{4}\left(\mathbb{T}^{2} \times \mathbb{C}^{2} \backslash Z^{\circ}\right)=\mathcal{I}_{1} .
\end{aligned}
$$

Proof. We consider three filtrations $\mathcal{V}, \mathcal{V}^{\prime}, \mathcal{V}^{\prime \prime}$ on $H^{4}\left(\mathbb{T}^{2} \times \mathbb{C}^{2} \backslash Z^{\circ}\right)$ and compare them. First, we define

$$
\mathcal{V}_{k} \Omega_{\mathbf{V}}^{i}(\log (Z+\mathbb{D})):=\Omega_{\mathbf{V}}^{i-k} \wedge \Omega_{\mathbf{V}}^{k}(\log (Z+\mathbb{D})), \quad(0 \leq k \leq 4) .
$$

This induces the weight filtration $\mathcal{V}_{k} H^{i}\left(\mathbb{T}^{2} \times \mathbb{C}^{2} \backslash Z^{\circ}\right)=\mathcal{W}_{k+4} H^{i}\left(\mathbb{T}^{2} \times \mathbb{C}^{2} \backslash\right.$ $Z^{\circ}$ ). We have already computed the dimension of graded quotients in Proposition A.5.

Second, let $U:=\mathbf{V} \backslash\left(Z \cup \mathbb{E}_{3} \cup \mathbb{E}_{4}\right)$. We define

$$
\mathcal{V}_{k}^{\prime} \Omega_{U}^{i}(\log (U \cap \mathbb{D})):=\Omega_{U}^{i-k} \wedge \Omega_{U}^{k}(\log (U \cap \mathbb{D})) \quad(k=0,1,2) .
$$

This induces another filtration $\mathcal{V}^{\prime}$ on $H^{4}\left(\mathbb{T}^{2} \times \mathbb{C}^{2} \backslash Z^{\circ}\right)$. As in [5, Section 8], this is given by the $\mathcal{I}$-filtration on $\mathcal{R}_{F}$ :

$$
\begin{aligned}
& \mathcal{V}_{0}^{\prime} H^{4}\left(\mathbb{T}^{2} \times \mathbb{C}^{2} \backslash Z^{\circ}\right) \cong \mathcal{I}_{1}, \quad \mathcal{V}_{1}^{\prime} H^{4}\left(\mathbb{T}^{2} \times \mathbb{C}^{2} \backslash Z^{\circ}\right) \cong \mathcal{I}_{3}, \\
& \mathcal{V}_{2}^{\prime} H^{4}\left(\mathbb{T}^{2} \times \mathbb{C}^{2} \backslash Z^{\circ}\right) \cong \mathcal{I}_{4} .
\end{aligned}
$$

Third, let $j: U \rightarrow \mathbf{V}$ be the inclusion and define

$$
\begin{aligned}
& \mathcal{V}_{k}^{\prime \prime} \Omega_{\mathbf{V}}^{i}(\log (Z+\mathbb{D})):=\Omega_{\mathbf{V}}^{i-k} \wedge \Omega_{\mathbf{V}, \mathbb{D}}^{k} \\
& \quad+\left(\mathcal{V}_{k-1}^{\prime} j_{*} \Omega_{U}^{i}(\log (U \cap \mathbb{D}))\right) \cap \Omega_{\mathbf{V}}^{i}(\log (Z+\mathbb{D})) .
\end{aligned}
$$

Since $H^{4}\left(\mathbb{T}^{2} \times \mathbb{C}^{2}\right)=0$, the first term does not contribute to $H^{4}\left(\mathbb{T}^{2} \times \mathbb{C}^{2} \backslash\right.$ $Z^{\circ}$ ). So the induced filtration is related to $\mathcal{V}^{\prime}$ by

$$
\mathcal{V}_{k}^{\prime \prime} H^{4}\left(\mathbb{T}^{2} \times \mathbb{C}^{2} \backslash Z^{\circ}\right)=\mathcal{V}_{k-1}^{\prime} H^{4}\left(\mathbb{T}^{2} \times \mathbb{C}^{2} \backslash Z^{\circ}\right), \quad(k=1,2,3) .
$$

Moreover, it holds that

$$
\begin{aligned}
& \mathcal{V}_{k} \Omega_{\mathbf{V}}^{i}(\log (Z+\mathbb{D})) \subset \mathcal{V}_{k}^{\prime \prime} \Omega_{\mathbf{V}}^{i}(\log (Z+\mathbb{D})), \quad(k=1,2), \\
& \mathcal{V}_{4} \Omega_{\mathbf{V}}^{i}(\log (Z+\mathbb{D})) \subset \mathcal{V}_{3}^{\prime \prime} \Omega_{\mathbf{V}}^{i}(\log (Z+\mathbb{D})) .
\end{aligned}
$$


Therefore, we have

$$
\begin{aligned}
& \mathcal{V}_{k} H^{4}\left(\mathbb{T}^{2} \times \mathbb{C}^{2} \backslash Z^{\circ}\right) \subset \mathcal{V}_{k-1}^{\prime} H^{4}\left(\mathbb{T}^{2} \times \mathbb{C}^{2} \backslash Z^{\circ}\right)(k=1,2), \\
& \mathcal{V}_{4} H^{4}\left(\mathbb{T}^{2} \times \mathbb{C}^{2} \backslash Z^{\circ}\right) \subset \mathcal{V}_{2}^{\prime} H^{4}\left(\mathbb{T}^{2} \times \mathbb{C}^{2} \backslash Z^{\circ}\right) .
\end{aligned}
$$

By the dimension consideration, we see that the proposition holds.

Since taking the residue map $H^{4}\left(\mathbb{T}^{2} \times \mathbb{C}^{2} \backslash Z^{\circ}\right) \rightarrow H^{3}\left(Z^{\circ}\right)$ decreases the weight by 2 , we obtain

Theorem A.2. The weight filtration on $H^{3}\left(Z^{\circ}\right)$ is as follows:

$$
\begin{aligned}
& \mathcal{W}_{6} H^{3}\left(Z^{\circ}\right) \cong \mathcal{R}_{F}, \\
& \mathcal{W}_{5} H^{3}\left(Z^{\circ}\right)=\mathcal{W}_{4} H^{3}\left(Z^{\circ}\right) \cong \mathcal{I}_{3}, \\
& \mathcal{W}_{3} H^{3}\left(Z^{\circ}\right) \cong \mathcal{I}_{1} .
\end{aligned}
$$

\section{A.5 Deformation and obstruction}

By Kawamata's result [27], $\left.H^{1}\left(Z, T_{Z}(-\log D)\right)\right)$ and $\left.H^{2}\left(Z, T_{Z}(-\log D)\right)\right)$ are the set of infinitesimal logarithmic deformations and the set of obstructions, respectively.

Let $\omega$ be the following global section of $K_{Z}(D)=\Omega_{Z}^{3}(\log D)$ :

$$
\omega=\operatorname{Res}_{Z} \frac{1}{P} \frac{d t_{1}}{t_{1}} \frac{d t_{2}}{t_{2}} d x d y
$$

\section{Proposition A.7.}

$$
\begin{aligned}
& H^{1}\left(Z, T_{Z}(-\log D)\right) \stackrel{\sim}{\rightarrow} H^{1}\left(Z, \Omega_{Z}^{2}(\log D)\right) \cong R_{F}^{1}, \\
& H^{2}\left(Z, T_{Z}(-\log D)\right) \stackrel{\sim}{\rightarrow} H^{2}\left(Z, \Omega_{Z}^{2}(\log D)\right)=0,
\end{aligned}
$$

where the isomorphisms are given by the contraction with the 3-form $\omega$.

Proof. The contraction with $\omega$ is an isomorphism since $K_{Z}(D) \cong \mathcal{O}_{Z}$ and $T_{Z}(-\log D)$ and $\Omega_{Z}^{2}(\log D)$ are locally free. For the rest, see Theorem A.1 and Remark A.1. 


\section{A.6 Variation of MHSs}

Varying the parameter $a \in \mathbb{L}_{\mathrm{reg}}(\Delta)$, we obtain a family of threefolds $Z_{a}^{\circ}$ :

$$
p^{\prime}: \mathcal{Z}^{\prime} \rightarrow \mathbb{L}_{\mathrm{reg}}(\Delta)
$$

We have

$$
R^{3} p_{*}^{\prime} \mathbb{Z} \otimes \mathcal{O}_{\mathbb{L}_{\mathrm{reg}}(\Delta)} \cong \mathcal{R}_{F}[a] \otimes \mathcal{O}_{\mathbb{L}_{\mathrm{reg}}(\Delta)}
$$

The Gauss-Manin connection on $\nabla_{a_{m}}$ on $R^{3} p_{*}^{\prime} \mathbb{Z} \otimes \mathcal{O}_{\mathbb{L}_{\mathrm{reg}}(\Delta)}$ corresponds to the derivation $\mathcal{D}_{a_{m}}$ since it corresponds to the differentiation by $a_{m}$ on $\Gamma \Omega_{\mathbb{T}^{2} \times \mathbb{C}^{2}}^{4}\left(* Z_{a}^{\circ}\right)$.

Let $\omega_{a}$ be the relative holomorphic 3 -form on $\mathcal{Z}^{\prime}$, such that

$$
\omega_{a}=\operatorname{Res} \frac{1}{F_{a}+x y} \frac{d t_{1}}{t_{1}} \frac{d t_{2}}{t_{2}} d x d y .
$$

By Proposition 3.1, we obtain

Corollary A.2. 1. $H^{3}\left(Z_{a}^{\circ}\right)$ is spanned by $\omega_{a}, \nabla_{\partial_{a_{m}}} \omega_{a}$ and $\nabla_{\partial_{a_{m}}} \nabla_{\partial_{a_{n}}} \omega_{a}$ $(m, n \in A(\Delta))$.

2. $\omega_{a}$ satisfies the A-hypergeometric system (3.8) with $\partial_{a_{i}}$ replaced by $\nabla_{\partial_{a_{i}}}$. 3. Period integrals of $\omega_{a}$ satisfies the A-hypergeometric system (3.8). Conversely, a solution of the A-hypergeometric system (3.8) is a period integral.

\section{Acknowledgments}

This work was started during Y.K.'s visit at Institut des Hautes Études Scientifiques. She thanks the institute for hospitality and support. She also thanks Maxim Kontsevich, Kyoji Saito, Akihiro Tsuchiya for valuable comments. The authors thank Andrea Brini for kindly letting them know some references on the Yukawa coupling. Research of Y.K. is partly supported by Grant-in-Aid for Scientific Research (Start-up 20840024). S.M. is supported by JSPS/EPDI/IHÉS fellowship (2007/2009) and Max-Planck-Instutut für Mathematik.

\section{References}

[1] M. Aganagic, A. Klemm, M. Marino and C. Vafa, The topological vertex, Comm. Math. Phys. 254(2) (2005), 425-478.

[2] M. Aganagic, V. Bouchard and A. Klemm, Topological strings and (almost) modular forms, Comm. Math. Phys. 277(3) (2008), 771-819. 
[3] M. Alim, J. D. Länge and P. Mayr, Global properties of topological string amplitudes and orbifold invariants, J. High Energy Phys. 113(3) (2010), 30 pp.

[4] V. I. Arnol'd, S. M. Gusein-Zade and A. N. Varchenko, Singularities of differentiable maps, Vol. II. Monodromy and asymptotics of integrals, Translated from the Russian by Hugh Porteous. Translation revised by the authors and James Montaldi (Monographs in Mathematics, 83), Birkhäuser Boston, Inc., Boston, MA, USA, 1988, viii+492 pp.

[5] V. V. Batyrev, Variations of the mixed Hodge structure of affine hypersurfaces in algebraic tori, Duke Math. J. 69(2) (1993), 349-409.

[6] __ Dual polyhedra and mirror symmetry for Calabi-Yau hypersurfaces in toric varieties, J. Algebraic Geom. 3(3) (1994), 493-535.

[7] M. Bershadsky, S. Cecotti, H. Ooguri and C. Vafa, Kodaira-Spencer theory of gravity and exact results for quantum string amplitudes, Comm. Math. Phys. 165(2) (1994), 311-427.

[8] A. Brini and A. Tanzini, Exact results for topological strings on resolved $Y^{p, q}$ singularities, Comm. Math. Phys. 289(1) (2009), 205-252.

[9] P. Candelas, X. C. de la Ossa, P. S. Green and L. Parkes, A pair of Calabi-Yau manifolds as an exactly soluble superconformal theory, Nuclear Phys. B359(1) (1991), 21-74.

[10] J. A. Carlson, Extensions of mixed Hodge structures, Journées de Géometrie Algébrique d'Angers, Juillet 1979, Sijthoff \& Noordhoff, Alphen an den Rijn-Germantown, Md., 1980, pp. 107-127.

[11] T.-M. Chiang, A. Klemm, S.-T. Yau and E. Zaslow, Local mirror symmetry: calculations and interpretations, Adv. Theor. Math. Phys. 3(3) (1999), 495-565.

[12] D. A. Cox and S. Katz, Mirror symmetry and algebraic geometry, Mathematical Surveys and Monographs, 68, American Mathematical Society, Providence, RI, 1999, pp. xxii+469

[13] P. Deligne, Théorie de Hodge. II. Inst. Hautes Études Sci. Publ. Math. 40 (1971), 5-57.

[14] , Théorie de Hodge. III. Inst. Hautes Études Sci. Publ. Math. 44 (1974), 5-77.

[15] B. Forbes and M. Jinzenji, Extending the Picard-Fuchs system of local mirror symmetry, J. Math. Phys. 46(8) (2005), 082302, 39 pp.

[16] I. M. Gel'fand, A. V. Zelevinskii and M. M. Kapranov, Hypergeometric functions and toric varieties. (Russian) Funktsional. Anal. i Prilozhen. 23(2) (1989), 12-26; translation in Funct. Anal. Appl. 23(2) (1989), 94-106. 
[17] , Generalized Euler integrals and A-hypergeometric functions, Adv. Math. 84(2) (1990), 255-271.

[18] B. R. Greene and M.R. Plesser, Duality in Calabi-Yau moduli space, Nuclear Phys. B 338(1) (1990), 15-37.

[19] P. Griffiths and J. Harris, Principles of algebraic geometry, Reprint of the 1978 original. Wiley Classics Library, John Wiley \& Sons, Inc., New York, 1994, pp. xiv+813.

[20] M. Gross, Examples of special Lagrangian fibrations, Symplectic geometry and mirror symmetry (Seoul, 2000). World Sci. Publ., River Edge, NJ, USA, 2001, pp. 81-109.

[21] A. Grothendieck, On the de Rham cohomology of algebraic varieties, Inst. Hautes Études Sci. Publ. Math. 29 (1966), 95-103.

[22] B. Haghighat, A. Klemm and M. Rauch, Integrability of the holomorphic anomaly equations, J. High Energy Phys. 097(10) (2008), 37 pp.

[23] K. Hori, A. Iqbal and C. Vafa, D-branes and mirror symmetry, arXiv:hep-th/0005247.

[24] S. Hosono, Counting BPS states via holomorphic anomaly equations, Calabi-Yau varieties and mirror symmetry (Toronto, ON, 2001), Fields Inst. Commun., 38, Amer. Math. Soc., Providence, RI, USA, 2003, pp. $57-86$.

[25] _ Central charges, symplectic forms, and hypergeometric series in local mirror symmetry, Mirror symmetry. V, AMS/IP Stud. Adv. Math., 38, Amer. Math. Soc., Providence, RI, USA, 2006, pp. 405-439.

[26] S. Hosono, A. Klemm, S. Theisen and S.-T. Yau, Mirror symmetry, mirror map and applications to Calabi-Yau hypersurfaces, Comm. Math. Phys. 167(2) (1995), 301-350.

[27] Y. Kawamata, On deformations of compactifiable complex manifolds, Math. Ann. 235(3) (1978), 247-265.

[28] S. H. Katz, A. Klemm and C. Vafa, Geometric engineering of quantum field theories, Nuclear Phys. B497 (1997), 173-195.

[29] A. Klemm and E. Zaslow, Local mirror symmetry at higher genus, Winter School on Mirror Symmetry, Vector Bundles and Lagrangian Submanifolds (Cambridge, MA, 1999), AMS/IP Stud. Adv. Math. 23, Amer. Math. Soc., Providence, RI, USA, 2001, pp. 183-207.

[30] A. R. Mavlyutov, Cohomology of rational forms and a vanishing theorem on toric varieties, J. Reine Angew. Math. 615 (2008), 45-58.

[31] T. Oda, Convex bodies and algebraic geometry. An introduction to the theory of toric varieties, Translated from the Japanese, Ergebnisse der Mathematik und ihrer Grenzgebiete 15(3), Springer-Verlag, Berlin, 1988, pp. viii+212. 
[32] C. A. M. Peters and J. H. M. Steenbrink, Mixed Hodge structures, Ergebnisse der Mathematik und ihrer Grenzgebiete, 52(3), Folge, Springer-Verlag, Berlin, 2008, pp. xiv +470 .

[33] J. Stienstra, Resonant hypergeometric systems and mirror symmetry, Integrable systems and algebraic geometry (Kobe/Kyoto, 1997), World Scientific Publishers, River Edge, NJ, USA, 1998, pp. $412-452$.

[34] N. Takahashi, Log mirror symmetry and local mirror symmetry, Comm. Math. Phys. 220(2) (2001), 293-299.

[35] S. Usui, Variation of mixed Hodge structures arising from family of logarithmic deformations, Ann. Sci. École Norm. Sup. (4) 16(1) (1983), 91-107.

[36] C. Voisin, Mirror symmetry, Translated from the 1996 French original by Roger Cooke. SMF/AMS Texts and Monographs, 1, American Mathematical Society, Providence, RI, Société Mathématique de France, Paris, 1999, pp. xx+120.

[37] _ Hodge theory and complex algebraic geometry, I, Translated from the French original by Leila Schneps. (Cambridge Studies in Advanced Mathematics, 76), Cambridge University Press, Cambridge, 2002.

[38] _ Hodge theory and complex algebraic geometry, II, Translated from the French original by Leila Schneps. (Cambridge Studies in Advanced Mathematics, 77), Cambridge University Press, Cambridge, 2003.

[39] E. Witten, Quantum background independence in string theory, arXiv:hep-th/9306122.

[40] S. Yamaguchi and S.-T. Yau, Topological string partition functions as polynomials, J. High Energy Phys. 047(7) (2004), 20 pp. (electronic). 
